\title{
On the Dimension of Posets with Cover Graphs of Treewidth 2
}

\author{
Gwenaël Joret $^{1}$ - Piotr Micek ${ }^{2}$ - William T. Trotter ${ }^{3}$. \\ Ruidong Wang ${ }^{3}$. Veit Wiechert ${ }^{4}$
}

Received: 16 April 2015 / Accepted: 13 May 2016 / Published online: 1 June 2016

(C) The Author(s) 2016. This article is published with open access at Springerlink.com

\begin{abstract}
In 1977, Trotter and Moore proved that a poset has dimension at most 3 whenever its cover graph is a forest, or equivalently, has treewidth at most 1. On the other hand, a wellknown construction of Kelly shows that there are posets of arbitrarily large dimension whose cover graphs have treewidth 3 . In this paper we focus on the boundary case of treewidth 2. It was recently shown that the dimension is bounded if the cover graph is outerplanar (Felsner, Trotter, and Wiechert) or if it has pathwidth 2 (Biró, Keller, and Young). This can be interpreted as evidence that the dimension should be bounded more generally when the cover graph has treewidth 2 . We show that it is indeed the case: Every such poset has dimension at most 1276.
\end{abstract}

Keywords Poset · Dimension · Treewidth

G. Joret was supported by a DECRA Fellowship from the Australian Research Council.

P. Micek is supported by the Mobility Plus program from The Polish Ministry of Science and higher Education.

V. Wiechert is supported by the Deutsche Forschungsgemeinschaft within the research training group 'Methods for Discrete Structures' (GRK 1408).

Piotr Micek

Piotr.Micek@tcs.uj.edu.pl

1 Computer Science Department, Université Libre de Bruxelles, Brussels, Belgium

2 Theoretical Computer Science Department, Faculty of Mathematics and Computer Science, Jagiellonian University, Kraków, Poland

3 School of Mathematics, Georgia Institute of Technology, Atlanta, Georgia 30332, USA

4 Institut für Mathematik, Technische Universität, Berlin, Berlin, Germany 


\section{Introduction}

The purpose of this paper is to show the following:

Theorem 1 Every poset whose cover graph has treewidth at most 2 has dimension at most 1276.

Let us provide some context for our theorem. Already in 1977, Trotter and Moore [11] showed that if the cover graph of a poset $P$ is a forest then $\operatorname{dim}(P) \leqslant 3$ and this is best possible, where $\operatorname{dim}(P)$ denotes the dimension of $P$. Recalling that forests are exactly the graphs of treewidth at most 1 , it is natural to ask how big can the dimension be for larger treewidths. Motivated by this question, we proceed with a brief survey of relevant results about the dimension of posets and properties of their cover graphs.

One such result, due to Felsner, Trotter and Wiechert [3], states that if the cover graph of a poset $P$ is outerplanar then $\operatorname{dim}(P) \leqslant 4$. Again, the bound is best possible. Note that outerplanar graphs have treewidth at most 2. Note also that one cannot hope for a similar bound on the dimension of posets with a planar cover graph. Indeed, already in 1981 Kelly [6] presented a family of posets $\left\{Q_{n}\right\}_{n} \geqslant 2$ with planar cover graphs and $\operatorname{dim}\left(Q_{n}\right)=n$ (see Fig. 1). One interesting feature of Kelly's construction for our purposes is that the cover graphs also have treewidth at most 3 (with equality for $n \geqslant 5$ ), as is easily verified. In fact, they even have pathwidth at most 3 (with equality for $n \geqslant 4$ ).

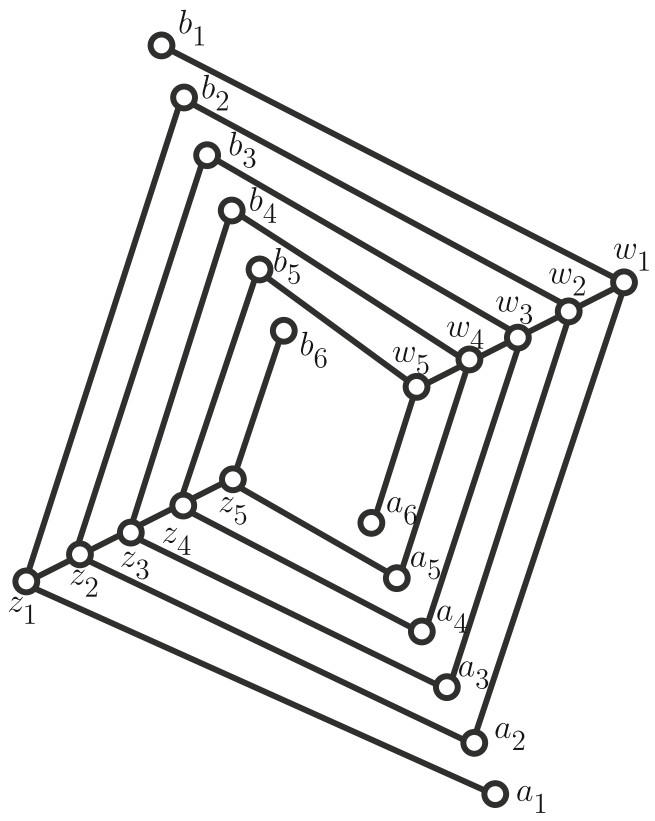

Fig. 1 Kelly's construction of a poset $Q_{n}$ with a planar cover graph containing the standard example $S_{n}$ as a subposet, for $n=6$. (Let us recall that the standard example $S_{n}$ is the poset on $2 n$ elements consisting of $n$ minimal elements $a_{1}, \ldots, a_{n}$ and $n$ maximal elements $b_{1}, \ldots, b_{n}$ which is such that $a_{i}<b_{j}$ in $S_{n}$ if and only if $i \neq j$.) The subposet induced by the $a_{i}$ 's and $b_{i}$ 's form $S_{6}$, which has dimension 6 . The general definition of $Q_{n}$ for any $n \geqslant 2$ is easily inferred from the figure. Since the standard example $S_{n}$ has dimension $n$, this shows that posets with planar cover graphs have unbounded dimension 
Very recently, Biró, Keller and Young [1] showed that if the cover graph of a poset $P$ has pathwidth at most 2, then its dimension is bounded: it is at most 17 . Furthermore, they proved that the treewidth of the cover graph of any poset containing the standard example $S_{n}$ with $n \geqslant 5$ is at least 3, thus showing in particular that Kelly's construction cannot be modified to have treewidth 2 .

To summarize, while the dimension of posets with cover graphs of treewidth 3 is unbounded, no such property is known to hold for the case of treewidth 2, and we cannot hope to obtain it by constructing posets containing large standard examples. Moreover, as mentioned above, the dimension is bounded for two important classes of graphs of treewidth at most 2, outerplanar graphs and graphs of pathwidth at most 2. All this can be interpreted as strong evidence that the dimension should be bounded more generally when the cover graph has treewidth at most 2 , which is exactly what we prove in this paper.

We note that the bound on the dimension we obtain is large (1276), and is most likely far from the truth. Furthermore, while we strove to make our arguments as simple as possibleand as a result did not try to optimize the bound - the proofs are lengthy and technical. We believe that there is still room for improvements, and it could very well be that a different approach would give a better bound and/or more insight into these problems.

We conclude this introduction by briefly mentioning a related line of research. Recently, new bounds for the dimension were found for certain posets of bounded height. Streib and Trotter [8] proved that for every positive integer $h$, there is a constant $c$ such that if a poset $P$ has height at most $h$ and its cover graph is planar, then $\operatorname{dim}(P) \leqslant c$. Joret, Micek, Milans, Trotter, Walczak, and Wang [4] showed that for every positive integers $h$ and $t$, there is a constant $c$ so that if $P$ has height at most $h$ and the treewidth of its cover graph is at most $t$, then $\operatorname{dim}(P) \leqslant c$.

These two results are closely related. In particular, one can deduce the result for planar cover graphs from the result for bounded treewidth cover graphs using a 'trick' introduced in [8] that reduces the problem to the special case where there is a special minimal element $a_{0}$ in the poset that is smaller than all the maximal elements. This implies that the diameter of the cover graph is bounded from above by a function of the height of the poset, and it is well-known that planar graphs with bounded diameter have bounded treewidth (see for instance [2]). This trick of having a special minimal element $a_{0}$ below all maximal elements turned out to be very useful in the context of this paper as well (though for different reasons), see Observation 6 in Section 2.

Finally, we mention that several new results on bounding the dimension of certain posets in terms of their height have recently been obtained [5, 7, 12], the interested reader is referred to [5] for a detailed overview of that area.

The paper is organized as follows. In Section 2 we give the necessary definitions and present a number of reductions, culminating in a more technical version of our theorem, Theorem 7. Then, in Section 3, we prove the result.

\section{Definitions and Preliminaries}

Let $P=(X, \leqslant)$ be a finite poset. The cover graph of $P$, denoted $\operatorname{cover}(P)$, is the graph on the elements of $P$ where two distinct elements $x, y$ are adjacent if and only if they are in a cover relation in $P$; that is, either $x<y$ or $x>y$ in $P$, and this relation cannot be deduced from transitivity. Informally, the cover graph of $P$ can be thought of as its order diagram seen as an undirected graph. The dimension of $P$, denoted $\operatorname{dim}(P)$, is the least positive integer $d$ for which there are $d$ linear extensions $L_{1}, \ldots, L_{d}$ of $P$ so that $x \leqslant y$ in $P$ if and 
only if $x \leqslant y$ in $L_{i}$ for each $i \in\{1, \ldots, d\}$. We mention that an introduction to the theory of posets and their dimension can be found in the monograph [9] and in the survey article [10].

When $x$ and $y$ are distinct elements in $P$, we write $x \| y$ to denote that $x$ and $y$ are incomparable. Also, we let $\operatorname{Inc}(P)=\{(x, y) \mid x, y \in X$ and $x \| y$ in $P\}$ denote the set of ordered pairs of incomparable elements in $P$. We denote by $\min (P)$ the set of minimal elements in $P$ and by $\max (P)$ the set of maximal elements in $P$. The downset of a set $S \subseteq X$ of elements is defined as $D(S)=\{x \in X \mid \exists s \in S$ such that $x \leqslant s$ in $P\}$, and similarly we define the upset of $S$ to be $U(S)=\{x \in X \mid \exists s \in S$ such that $\leqslant x$ in $P\}$.

A set $I \subseteq \operatorname{Inc}(P)$ of incomparable pairs is reversible if there is a linear extension $L$ of $P$ with $x>y$ in $L$ for every $(x, y) \in I$. It is easily seen that if $P$ is not a chain, then $\operatorname{dim}(P)$ is the least positive integer $d$ for which there exists a partition of $\operatorname{Inc}(P)$ into $d$ reversible sets.

A subset $\left\{\left(x_{i}, y_{i}\right)\right\}_{i=1}^{k}$ of $\operatorname{Inc}(P)$ with $k \geqslant 2$ is said to be an alternating cycle if $x_{i} \leqslant y_{i+1}$ in $P$ for each $i \in\{1,2, \ldots, k\}$, where indices are taken cyclically (thus $x_{k} \leqslant y_{1}$ in $P$ is required). For example, in the poset $Q_{6}$ of Fig. 1 the pairs $\left(a_{i}, b_{i}\right),\left(a_{j}, b_{j}\right)$ form an alternating cycle of length 2 for all $i, j \in\{1, \ldots, 6\}$ such that $i \neq j$. An alternating cycle $\left\{\left(x_{i}, y_{i}\right)\right\}_{i=1}^{k}$ is strict if, for each $i, j \in\{1,2, \ldots, k\}$, we have $x_{i} \leqslant y_{j}$ in $P$ if and only if $j=i+1$ (cyclically). Note that in that case $x_{1}, x_{2}, \ldots, x_{k}$ are all distinct, and $y_{1}, y_{2}, \ldots, y_{k}$ are all distinct. Notice also that every non-strict alternating cycle can be made strict by discarding some of its incomparable pairs.

Observe that if $I=\left\{\left(x_{i}, y_{i}\right)\right\}_{i=1}^{k}$ is an alternating cycle in $\operatorname{Inc}(P)$ then $I$ cannot be reversed by a linear extension $L$ of $P$. Indeed, otherwise we would have $y_{i}<x_{i} \leqslant y_{i+1}$ in $L$ for each $i \in\{1,2, \ldots, k\}$, which cannot hold cyclically. Hence, alternating cycles are not reversible. It is easily checked - and this was originally observed by Trotter and Moore [11] - that every non-reversible subset $I \subseteq \operatorname{Inc}(P)$ contains an alternating cycle, and thus a strict alternating cycle:

Observation 2 A set I of incomparable pairs of a poset $P$ is reversible if and only if I contains no strict alternating cycle.

An incomparable pair $(x, y)$ of a poset $P$ is said to be a min-max pair if $x$ is minimal in $P$ and $y$ is maximal in $P$. The set of all min-max pairs in $P$ is denoted by $\operatorname{MM}(P)$. Define $\operatorname{dim}^{*}(P)$ as the least positive integer $t$ such that $\operatorname{MM}(P)$ can be partitioned into $t$ reversible subsets if $\operatorname{MM}(P) \neq \emptyset$, and as being equal to 1 otherwise. For our purposes, when bounding the dimension we will be able to focus on reversing only those incomparable pairs that are min-max pairs. This is the content of Observation 3 below. In order to state this observation formally we first need to recall some standard definitions from graph theory.

By 'graph' we will always mean an undirected finite simple graph in this paper. The treewidth of a graph $G=(V, E)$ is the least positive integer $t$ such that there exist a tree $T$ and non-empty subtrees $T_{x}$ of $T$ for each $x \in V$ such that

(i) $V\left(T_{x}\right) \cap V\left(T_{y}\right) \neq \emptyset$ for each edge $x y \in E$, and

(ii) $\left|\left\{x \in V \mid u \in V\left(T_{x}\right)\right\}\right| \leqslant t+1$ for each node $u$ of the tree $T$.

The pathwidth of $G$ is defined as treewidth, except that the tree $T$ is required to be a path. A graph $H$ is a minor of a graph $G$ if $H$ can be obtained from a subgraph of $G$ by contracting edges. (We note that since we only consider simple graphs, loops and parallel edges resulting from edge contractions are deleted). Recall that the class of graphs of treewidth at most $k(k \geqslant 0)$ is closed under taking minors, thus $\operatorname{tw}(H) \leqslant \operatorname{tw}(G)$ for every graph $G$ and minor $H$ of $G$. 
Given a class $\mathcal{F}$ of graphs, we let $\widehat{\mathcal{F}}$ denote the class of graphs that can be obtained from a graph $G \in \mathcal{F}$ by adding independently for each vertex $v$ of $G$ zero, one, or two new pendant vertices adjacent to $v$. We will use the easy observation that $\widehat{\mathcal{F}}=\mathcal{F}$ when $\mathcal{F}$ is the class of graphs of treewidth at most $k$ (provided $k \geqslant 1$ ). We note that $\widehat{\mathcal{F}}=\mathcal{F}$ holds for other classes $\mathcal{F}$ of interest, such as planar graphs.

The next elementary observation is due to Streib and Trotter [8], who were interested in the case of planar cover graphs. We provide a proof for the sake of completeness.

Observation 3 Let $\mathcal{F}$ be a class of graphs. If $P$ is a poset with $\operatorname{cover}(P) \in \mathcal{F}$ then there exists a poset $Q$ such that

(i) $\operatorname{cover}(Q) \in \widehat{\mathcal{F}}$, and

(ii) $\operatorname{dim}(P) \leqslant \operatorname{dim}^{*}(Q)$.

Proof If $P$ is a chain then we set $Q=P$ and the statement can be easily verified.

Otherwise, let $Q$ be the poset constructed from $P$ as follows: For each non-minimal element $x$ of $P$, add a new element $x^{\prime}$ below $x$ (and its upset) such that $x^{\prime}<x$ is the only cover relation involving $x^{\prime}$ in $Q$. Also, for each non-maximal element $y$ of $P$, add a new element $y^{\prime \prime}$ above $y$ (and its downset) such that $y<y^{\prime \prime}$ is the only cover relation involving $y^{\prime \prime}$ in $Q$. Now, the cover graph of $Q$ is the same as the cover graph of $P$ except that we attached up to two new pendant vertices to each vertex.

For convenience, we also define an element $x^{\prime}$ for each minimal element $x$ of $P$, simply by setting $x^{\prime}=x$. Similarly, we let $y^{\prime \prime}=y$, for each maximal element $y$ of $P$.

Observe that if a set $\mathcal{L}$ of linear extensions of $Q$ reverses all min-max pairs of $Q$ then it must reverse all incomparable pairs of $P$. Indeed, for each pair $(x, y) \in \operatorname{Inc}(P)$ consider the min-max pair $\left(x^{\prime}, y^{\prime \prime}\right)$ in $Q$. There is some linear extension $L \in \mathcal{L}$ reversing $\left(x^{\prime}, y^{\prime \prime}\right)$. Given that $x^{\prime} \leqslant x$ and $y \leqslant y^{\prime \prime}$ in $Q$, it follows that $y \leqslant y^{\prime \prime}<x^{\prime} \leqslant x$ in L. Hence, restricting the linear orders in $\mathcal{L}$ to the elements of $P$ we deduce that $L$ reverses all pairs in $\operatorname{Inc}(P)$ so $\operatorname{dim}(P) \leqslant|\mathcal{L}|$ (as $P$ is not a chain). Therefore, $\operatorname{dim}(P) \leqslant \operatorname{dim}^{*}(Q)$.

As a corollary, for treewidth we obtain:

Observation 4 For every poset $P$ there exists a poset $Q$ such that

(i) $\operatorname{tw}(\operatorname{cover}(P))=\operatorname{tw}(\operatorname{cover}(Q))$, and

(ii) $\operatorname{dim}(P) \leqslant \operatorname{dim}^{*}(Q)$.

Proof This follows from Observation 3 if $\operatorname{tw}(\operatorname{cover}(P)) \geqslant 1$. If, on the other hand, $\operatorname{tw}(\operatorname{cover}(P))=0$, then $P$ is an antichain and we can simply take $Q=P$.

In the next observation we consider posets with disconnected cover graphs. As expected, we define the components of a poset $P$ as the subposets of $P$ induced by the components of its cover graph.

Observation 5 If $P$ is a poset with $k \geqslant 2$ components $C_{1}, \ldots, C_{k}$ then either

(i) $P$ is a disjoint union of chains and we have $\operatorname{dim}(P)=\operatorname{dim}^{*}(P)=2$, or

(ii) $\operatorname{dim}(P)=\max \left\{\operatorname{dim}\left(C_{i}\right) \mid i=1, \ldots, k\right\}$ and

$\operatorname{dim}^{*}(P)=\max \left\{\operatorname{dim}^{*}\left(C_{i}\right) \mid i=1, \ldots, k\right\}$. 
Proof If for each $i \in\{1, \ldots, k\}$ the subposet $C_{i}$ of $P$ is a chain then it is easy to see that $\operatorname{dim}(P)=\operatorname{dim}^{*}(P)=2$. Thus we may assume that this is not the case, that is, $\operatorname{dim}\left(C_{i}\right) \geqslant 2$ for some $i \in\{1, \ldots, k\}$.

For each $i \in\{1, \ldots, k\}$ let $\mathcal{R}_{i}$ be a family of $\operatorname{dim}\left(C_{i}\right)$ linear extensions of $C_{i}$ witnessing the dimension of $C_{i}$. We construct a family $\mathcal{R}$ of linear extensions of $P$ in the following way. First, let $\mathcal{R}:=\emptyset$. Then, as long as there is a set $\mathcal{R}_{i}$ which is not empty,

(i) choose a linear extension $L_{i} \in \mathcal{R}_{i}$ for each $i \in\{1, \ldots, k\}$ such that $\mathcal{R}_{i}$ is not empty;

(ii) choose any linear extension $L_{i}$ of $C_{i}$ for each $i \in\{1, \ldots, k\}$ such that $\mathcal{R}_{i}$ is empty;

(iii) add to $\mathcal{R}$ the linear extension $L$ of $P$ defined by $L:=L_{1}<L_{2}<\ldots<L_{k}$, and

(iv) remove $L_{i}$ from $\mathcal{R}_{i}$ for each $i \in\{1, \ldots, k\}$.

Clearly, $|\mathcal{R}|=\max \left\{\operatorname{dim}\left(C_{i}\right) \mid i \in\{1, \ldots, k\}\right\}$. Now consider one arbitrarily chosen linear extension $L \in \mathcal{R}$; say we had $L=L_{1}<\ldots<L_{k}$ when it was defined above, and replace $L$ by $L^{\prime}:=L_{k}<\ldots<L_{1}$ in $\mathcal{R}$. It is easy to verify that the resulting family $\mathcal{R}$ reverses all incomparable pairs in $P$. In particular, all incomparable pairs of $P$ with elements from distinct components are reversed by $L^{\prime}$ and any other linear extension in $\mathcal{R}$ (note there is at least one more as $\operatorname{dim}\left(C_{i}\right) \geqslant 2$ for some $\left.i\right)$. This shows that $\operatorname{dim}(P)=\max \left\{\operatorname{dim}\left(C_{i}\right) \mid i \in\right.$ $\{1, \ldots, k\}\}$.

The proof for $\operatorname{dim}^{*}(P)$ goes along the same lines and is thus omitted.

To prove the next observation we partition the minimal and maximal elements of a poset by 'unfolding' the poset from an arbitrary minimal element, and contract some part of the poset into a single element. This proof idea is due to Streib and Trotter [8], and is very useful for our purposes. In [8] it was used in the context of planar cover graphs but it works equally well for any minor-closed class of graphs.

Observation 6 For every poset $P$ there exists a poset $Q$ such that

(i) $\operatorname{cover}(Q)$ is a minor of $\operatorname{cover}(P)$ (and thus in particular $\operatorname{tw}(\operatorname{cover}(Q)) \leqslant$ $\operatorname{tw}(\operatorname{cover}(P)))$;

(ii) there is an element $q_{0} \in \min (Q)$ with $q_{0}<q$ in $Q$ for all $q \in \max (Q)$, and

(iii) $\operatorname{dim}^{*}(P) \leqslant 2 \operatorname{dim}^{*}(Q)$.

Proof First of all, we note that it is enough to prove the statement in the case where cover $(P)$ is connected. Indeed, if $\operatorname{cover}(P)$ is disconnected then by Observation 5 either $P$ is a disjoint union of chains and $\operatorname{dim}(P)=\operatorname{dim}^{*}(P)=2$, in which case the observation is trivial, or $\operatorname{dim}^{*}(P)=\max \left\{\operatorname{dim}^{*}(C) \mid C\right.$ component of $\left.P\right\}$ and we can simply consider a component $C$ of $P$ with $\operatorname{dim}^{*}(P)=\operatorname{dim}^{*}(C)$.

From now on we suppose that $\operatorname{cover}(P)$ is connected. We are going to build a small set of linear extensions of $P$ reversing all min-max pairs of $P$. Partition the minimal and maximal elements of $P$ as follows. Choose an arbitrary element $a_{0} \in \min (P)$, let $A_{0}=\left\{a_{0}\right\}$, and for $i=1,2,3, \ldots$ let

$$
\begin{aligned}
& B_{i}=\left\{b \in \max (P)-\bigcup_{1 \leqslant j<i} B_{j} \mid \text { there exists } a \in A_{i-1} \text { with } a<b \text { in } P\right\}, \\
& A_{i}=\left\{a \in \min (P)-\bigcup_{0 \leqslant j<i} A_{j} \mid \text { there exists } b \in B_{i} \text { with } a<b \text { in } P\right\} .
\end{aligned}
$$

Let $k$ be the least index such that $A_{k}$ is empty. See Fig. 2 for an illustration. The fact that each minimal and maximal element of $P$ is included in one of the sets defined above follows 

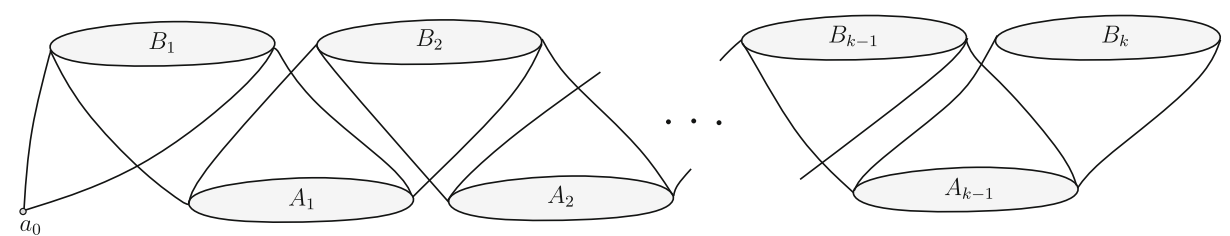

Fig. 2 Schematic drawing of $P$ and the sets $A_{0}, A_{1}, \ldots, A_{k-1}$ and $B_{1}, \ldots, B_{k}$

from the connectivity of $\operatorname{cover}(P)$. If $k=1$ then $a_{0}$ is below all maximal elements of $P$ and hence $P$ itself satisfies conditions (i)-(iii). So we may assume $k \geqslant 2$ from now on.

Let $Q_{i}^{i+1}$ be the poset on the set of elements $X_{i}^{i+1}=A_{i} \cup B_{i+1} \cup\left(U\left(A_{i}\right) \cap D\left(B_{i+1}\right)\right)$ with order relation inherited from $P$. Figure 3 illustrates this definition. Let

$$
t=\max \left\{\operatorname{dim}^{*}\left(Q_{i}^{i+1}\right) \mid i=0, \ldots, k-1\right\} .
$$

For each $i \in\{0, \ldots, k-1\}$ consider $t$ linear extensions $L_{1}^{i}, \ldots, L_{t}^{i}$ of $Q_{i}^{i+1}$ that reverse all pairs from the set $\mathrm{MM}\left(Q_{i}^{i+1}\right)$. Combining these we define $t$ linear extensions of $P$. For $j \in\{1, \ldots, t\}$ let $L_{j}$ be a linear extension of $P$ that contains the linear order

$$
L_{j}^{k-1}<\cdots<L_{j}^{1}<L_{j}^{0} .
$$

Then, $L_{1}, \ldots, L_{t}$ reverse all pairs $(a, b) \in \mathrm{MM}(P)$ with $a \in A_{i}$ and $b \in B_{j}$ where $j \geqslant i+1$. In a similar way we are able to reverse the pairs where $j \leqslant i$.

Let $Q_{i}^{i}$ be the poset on the set of elements $X_{i}^{i}=A_{i} \cup B_{i} \cup\left(U\left(A_{i}\right) \cap D\left(B_{i}\right)\right)$ being ordered as in $P$. We set $t^{\prime}=\max \left\{\operatorname{dim}^{*}\left(Q_{i}^{i}\right) \mid i \in\{1, \ldots, k-1\}\right\}$ and for each $i \in\{1, \ldots, k-1\}$ we fix $t^{\prime}$ linear extensions $L_{1}^{i}, \ldots, L_{t^{\prime}}^{i}$ of $Q_{i}^{i}$ reversing all pairs from $\operatorname{MM}\left(Q_{i}^{i}\right)$. Again, we combine these to obtain linear extensions of $P$. For $j \in\left\{1, \ldots, t^{\prime}\right\}$ let $L_{j}^{\prime}$ be a linear extension of $P$ that contains the linear order

$$
L_{j}^{1}<L_{j}^{2}<\cdots<L_{j}^{k-1} .
$$

Clearly, $L_{1}^{\prime} \ldots, L_{t^{\prime}}^{\prime}$ reverse all pairs $(a, b) \in \mathrm{MM}(P)$ with $a \in A_{i}$ and $b \in B_{j}$ where $j \leqslant i$. It follows that $L_{1}, \ldots, L_{t}, L_{1}^{\prime} \ldots, L_{t^{\prime}}^{\prime}$ reverse the set $\operatorname{MM}(P)$ and hence $\operatorname{dim}^{*}(P) \leqslant$ $t+t^{\prime}$.

Now suppose first $t>t^{\prime}$, so in particular $t>1$. Then let $\ell \in\{0, \ldots, k-1\}$ such that $t=\operatorname{dim}^{*}\left(Q_{\ell}^{\ell+1}\right)$. Note that we must have $\ell \geqslant 1$ since $\operatorname{dim}^{*}\left(Q_{0}^{1}\right)=1<t$. We define $Q$ to be the poset that is obtained from $Q_{\ell}^{\ell+1}$ by adding an extra element $q$ which is such that $q>x$ for all $x \in X_{\ell}^{\ell+1} \cap D\left(B_{\ell}\right)$, and incomparable to all other elements of $Q_{\ell}^{\ell+1}$ (here we need $\ell \geqslant 1$ so that $B_{\ell}$ exists). In particular, $q>a$ for all $a \in A_{\ell}$. Observe that
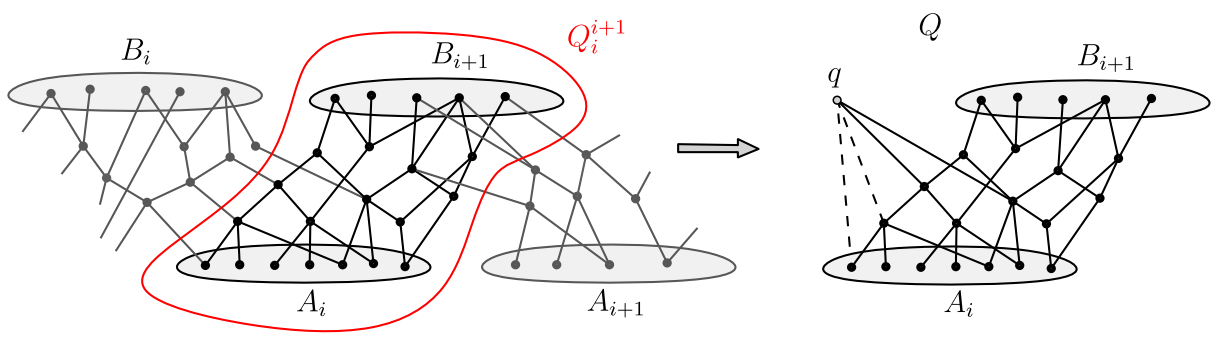

Fig. 3 Definition of $Q_{i}^{i+1}$ and construction of $Q$ with its cover graph 
the cover graph of $Q$ is an induced subgraph of $\operatorname{cover}(P)$ with an extra vertex $q$ linked to some of the other vertices. Here, $q$ can be seen as the result of the contraction of the connected set $\bigcup_{1 \leqslant j \leqslant \ell} D\left(B_{j}\right)-X_{\ell}^{\ell+1}$ plus the deletion of some of the edges incident to the contracted vertex (see Fig. 3 with $i=\ell$, dashed edges indicate deletions). The deletion step is necessary, as after the contraction it might be that some edges incident to $q$ do not correspond to cover relations anymore. It follows that $\operatorname{cover}(Q)$ is a minor of $\operatorname{cover}(P)$. Furthermore, it holds that

$$
\operatorname{dim}^{*}(P) \leqslant 2 t=2 \operatorname{dim}^{*}\left(Q_{\ell}^{\ell+1}\right) \leqslant 2 \operatorname{dim}^{*}(Q) .
$$

Therefore, the dual of $Q$ satisfies conditions (i)-(iii).

The case $t^{\prime} \geqslant t$ goes along similar lines as in the first case (with the slight difference that we do not need to exclude the subcase $t^{\prime}=1$ ). We leave the details to the reader.

Applying these observations we move from Theorem 1 to a more technical statement.

\section{Theorem 7 Let $P$ be a poset with}

(i) a cover graph of treewidth at most 2 , and

(ii) a minimal element $a_{0} \in \min (P)$ such that $a_{0}<b$ for all $b \in \max (P)$.

Then the set $\mathrm{MM}(P)$ can be partitioned into 638 reversible sets.

In order to deduce Theorem 1 from Theorem 7 consider any poset $P$ with cover graph of treewidth at most 2 . By Observation 4 there is a poset $Q$ with $\operatorname{tw}(\operatorname{cover}(Q)) \leqslant 2$ and $\operatorname{dim}$ $(P) \leqslant \operatorname{dim}^{*}(Q)$. Now by Observation 6 and applying Theorem 7 there is a poset $R$ with tw $(\operatorname{cover}(R)) \leqslant 2$, a minimal element $a_{0} \in \min (R)$ such that $a_{0}<b$ for all $b \in \max (R)$, and

$$
\operatorname{dim}(P) \leqslant \operatorname{dim}^{*}(Q) \leqslant 2 \operatorname{dim}^{*}(R) \leqslant 2 \cdot 638=1276,
$$

as desired.

From now on we focus on the proof of Theorem 7. Let $P=(X, \leqslant)$ be a poset fulfilling the conditions of Theorem 7. Consider a tree decomposition of width at most 2 of $\operatorname{cover}(P)$, consisting of a tree $T$ and subtrees $T_{x}$ for each $x \in X$. We may assume that the width of the decomposition is exactly 2 , since otherwise $\operatorname{dim}(P) \leqslant 3$ by the result of Trotter and Moore [11], and the theorem follows trivially.

For each node $u$ of $T$ let $B(u)$ denote its bag, namely, the set $\left\{x \in X \mid u \in V\left(T_{x}\right)\right\}$. Since the tree decomposition has width 2 , every bag has size at most 3 , and at least one bag has size exactly 3 . Modifying the tree decomposition if necessary, we may suppose that every bag has size 3. Indeed, say $u v$ is an edge of $T$ with $|B(u)|=3$ and $|B(v)| \leqslant 2$. Then choose arbitrarily $3-|B(v)|$ elements from $B(u) \backslash B(v)$ and add them to $B(v)$. Repeating this process as many times as necessary, we eventually ensure that every bag has size 3 . Note that the subtrees $T_{x}(x \in X)$ of the tree decomposition are uniquely determined by the bags, and vice versa; thus, it is enough to specify how $T$ and the bags are modified. The above modification repeatedly adds leaves to some of the subtrees $T_{x}(x \in X)$, which clearly keeps the fact that $T$ and the subtrees $T_{x}(x \in X)$ form a tree decomposition of $\operatorname{cover}(P)$.

Recall that, by the assumptions of Theorem 7, the poset $P$ has a minimal element $a_{0}$ with $a_{0}<b$ for all $b \in \max (P)$. This implies that the cover graph of $P$ is connected. Using this, we may suppose without loss of generality that $|B(u) \cap B(v)| \geqslant 1$ for each edge $u v$ of $T$. For if this does not hold, then the bags of one of the two components of $T-u v$ are all empty (as is easily checked), and thus the nodes of that component can be removed from $T$ without affecting the tree decomposition. 
In fact, we may even assume that $|B(u) \cap B(v)|=2$ holds for every edge $u v$ of $T$. To see this, consider the following iterative modification of the tree decomposition: Suppose that $u v$ is an edge of $T$ such that $t:=|B(u) \cap B(v)| \neq 2$. If $t=3$ then simply identify $u$ and $v$, and contract the edge $u v$ in $T$. If $t=1$ then subdivide the edge $u v$ in $T$ with a new node $w$, and let the bag $B(w)$ of $w$ be the set $(B(u) \cap B(v)) \cup\{x, y\}$, where $x$ and $y$ are arbitrarily chosen elements in $B(u) \backslash B(v)$ and $B(v) \backslash B(u)$, respectively. These modifications are valid, in the sense that the bags still define a tree decomposition of $\operatorname{cover}(P)$ of width 2 , and in order to ensure the desired property it suffices to apply them iteratively until there is no problematic edge left.

To summarize, in the tree decomposition we have $|B(u)|=3$ for every node $u$ of $T$, and $|B(u) \cap B(v)|=2$ for every edge $u v$ of $T$. We will need to further refine our tree decomposition so as to ensure a few extra properties. These changes will be explained one by one below. Let us mention that we will keep the fact that $|B(u) \cap B(v)|=2$ for every edge $u v$ of $T$, and that $|B(u)|=3$ for every internal node $u$ of $T$. However, we will add new leaves to $T$ having bags of size 2 only.

Choose an arbitrary node $r^{\prime} \in V(T)$ with $a_{0} \in B\left(r^{\prime}\right)$. Add a new node $r$ to $T$ and make it adjacent to $r^{\prime}$. The bag $B(r)$ of $r$ is defined as the union of $a_{0}$ and one arbitrarily chosen element from $B\left(r^{\prime}\right)-\left\{a_{0}\right\}$. (Observe that the size of $B(r)$ is only 2; on the other hand, we do have $\left|B(r) \cap B\left(r^{\prime}\right)\right|=2$.) We call $r$ the root of $T$, and thus see $T$ as being rooted at $r$. (For a technical reason we need the root to be a leaf of $T$, which explains why we set it up this way.) Every non-root node $u$ in $T$ has a parent $\mathrm{p}(u)$ in $T$, namely, the neighbor of $u$ on the path from $u$ to $r$ in $T$. Now we have an order relation on the nodes of $T$, namely $u \leqslant v$ in $T$ if $u$ is on the path from $r$ to $v$ in $T$. The following observation will be useful later.

Observation 8 If $v_{1}, \ldots, v_{n}$ is a sequence of nodes of $T$ such that consecutive nodes are comparable in $T$ (that is $v_{i} \leqslant v_{i+1}$ or $v_{i+1} \leqslant v_{i}$ in $T$ for each $i \in\{1, \ldots, n-1\}$ ), then there is an index $j \in\{1, \ldots, n\}$ such that $v_{j} \leqslant v_{i}$ in $T$ for each $i \in\{1, \ldots, n\}$.

Proof We prove this by induction on $n$. For $n=1$ it is immediate. So suppose that $n>1$. Then we can apply the induction hypothesis on the sequence $v_{1}, \ldots, v_{n-1}$ and get $j \in$ $\{1, \ldots, n-1\}$ such that $v_{j} \leqslant v_{i}$ for each $i \in\{1, \ldots, n-1\}$. As $v_{n-1}$ and $v_{n}$ are comparable in $T$, we have $v_{n-1} \leqslant v_{n}$ or $v_{n} \leqslant v_{n-1}$ in $T$. In the first case we conclude $v_{j} \leqslant v_{n-1} \leqslant v_{n}$ in $T$ and we are done. In the second case we have $\left\{v_{j}, v_{n}\right\} \leqslant v_{n-1}$ in $T$, which makes $v_{j}$ and $v_{n}$ comparable in $T$. But clearly, from this it follows that $v_{j} \leqslant v_{i}$ in $T$ for each $i \in\{1, \ldots, n\}$ or $v_{n} \leqslant v_{i}$ in $T$ for each $i \in\{1, \ldots, n\}$.

Fix a planar drawing of the tree $T$ with the root $r$ at the bottom. Suppose that $v$ and $v^{\prime}$ are two nodes of $T$ that are incomparable in $T$. Take the maximum node $u$ (with respect to the order in $T$ ) such that $u \leqslant v$ and $u \leqslant v^{\prime}$ in $T$. We denote this node by $v \wedge v^{\prime}$. Observe that $u$ has degree at least 2 in $T$, and hence is distinct from the root $r$. (Ensuring this is the reason why we made sure that the root $r$ is a leaf.) Consider the edge $p$ from $u$ to $\mathrm{p}(u)$, the edge $e$ from $u$ towards $v$ and the edge $e^{\prime}$ from $u$ towards $v^{\prime}$. All these edges are distinct. If the clockwise order around $u$ in the drawing is $p, e, e^{\prime}$ for these three edges, then we say that $v$ is to the left of $v^{\prime}$ in $T$, otherwise the clockwise order around $u$ is $p, e^{\prime}, e$ and we say that $v$ is to the right of $v^{\prime}$ in $T$. Observe that the relations "is left of in $T$ " and "is right of in $T$ " both induce a linear order on any set of nodes which are pairwise incomparable in $T$.

Observation 9 Let $v$ and $v^{\prime}$ be incomparable nodes in $T$ with $v$ left of $v^{\prime}$ in $T$, and let $u:=v \wedge v^{\prime}$. If $w$ and $w^{\prime}$ are the neighbors of $u$ on the paths towards $v$ and $v^{\prime}$ in $T$, respectively, then for each node $c$ in $T$ we have that 


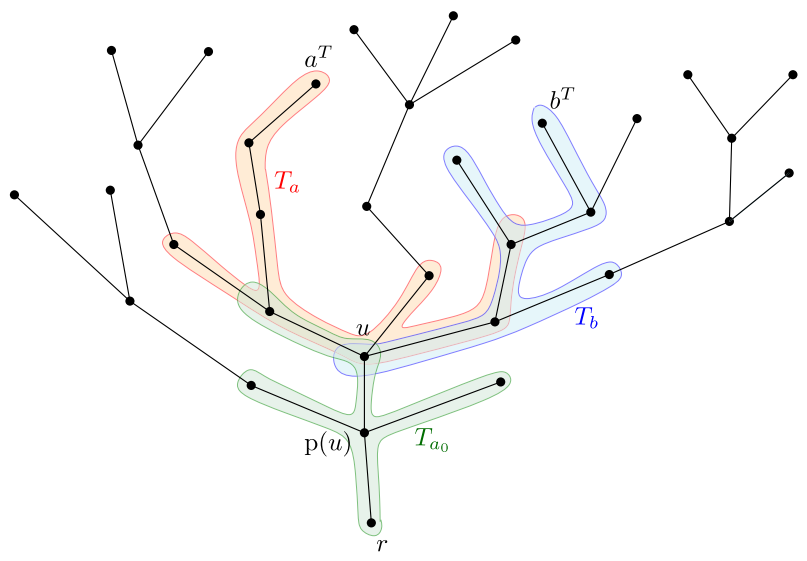

Fig. 4 We have the following properties in this example: $(a, b) \in \operatorname{MM}(P)$ with $a^{T}$ left of $b^{T}$ in $T$, and $u=a^{T} \wedge b^{T}$ (and hence $u<a^{T}$ and $u<b^{T}$ in $T$ ). We also have $B(u)=\left\{a_{0}, a, b\right\}$ here

(i) $v$ is left of $c$ in $T$ if $w^{\prime} \leqslant c$ in $T$, and

(ii) $c$ is left of $v^{\prime}$ in $T$ if $w \leqslant c$ in $T$.

Proof If $w^{\prime} \leqslant c$ in $T$, then we also have $u=v \wedge c$, and the first edge on the path from $u$ to $c$ in $T$ is the same as that of the path from $u$ to $v^{\prime}$ in $T$. Since $v$ is left of $v^{\prime}$ in $T$, it follows that $v$ is left of $c$ as well. The proof for the second item is analogous.

Next we modify once more the tree decomposition. For each element $a \in \min (X)$ such that $(a, b) \in \operatorname{MM}(P)$ for some $b \in \max (X)$, choose arbitrarily a node $w_{a}$ of $T$ such that $a \in B\left(w_{a}\right)$. Similarly, for each element $b \in \max (X)$ such that $(a, b) \in \operatorname{MM}(P)$ for some $a \in \min (X)$, choose arbitrarily a node $w_{b}$ of $T$ such that $b \in B\left(w_{b}\right)$. (Note that the same node of $T$ could possibly be chosen more than once.) Now that all these choices are made, for each minimal element $a$ of $P$ considered above, add a new leaf $a^{T}$ to $T$ adjacent to $w_{a}$ with bag $B\left(a^{T}\right):=\{a, x\}$, where $x$ is an arbitrarily chosen element from $B\left(w_{a}\right) \backslash\{a\}$. Similarly, for each maximal element $b$ of $P$ considered above, add a new leaf $b^{T}$ to $T$ adjacent to $w_{b}$ with bag $B\left(b^{T}\right):=\{b, x\}$, where $x$ is an arbitrarily chosen element from $B\left(w_{b}\right) \backslash\{b\}$.

This concludes our modifications of the tree decomposition. Notice that we made sure that $|B(u)|=3$ for every internal node $u$ of $T$, and that $|B(u) \cap B(v)|=2$ for every edge $u v$ of $T$. Observe also that for every pair $(a, b) \in \mathrm{MM}(P)$, the two nodes $a^{T}$ and $b^{T}$ are incomparable in $T$, and thus one is to the left of the other in $T$. Figure 4 provides an illustration. (We also note that while the tree $T$ has been modified since stating Observations 8 and 9 , they obviously still apply to the new tree $T$ ).

Let $G$ be the intersection graph of the subtrees $T_{x}(x \in X)$ of $T$. Thus two distinct elements $x, y \in X$ are adjacent in $G$ if and only if $V\left(T_{x}\right) \cap V\left(T_{y}\right) \neq \emptyset$. The graph $G$ is chordal and the maximum clique size in $G$ is 3 . Hence the vertices of $G$ can be (properly) colored with three colors. We fix a 3-coloring $\phi$ of $X$ which is such that $x, y \in X$ receive distinct colors whenever $V\left(T_{x}\right) \cap V\left(T_{y}\right) \neq \emptyset$. In particular, if $x$ and $y$ are two distinct elements of $P$ such that $x, y \in B(u)$ for some $u \in V(T)$ then $x$ and $y$ receive different colors. 
We end this section with a fundamental observation which is going to be used repeatedly in a number of forthcoming arguments. We say that a relation $x \leqslant y$ in $P$ hits a set $Z \subseteq X$ if there exists $z \in Z$ with $x \leqslant z \leqslant y$ in $P$.

Observation 10 Let $x \leqslant y$ in $P$ and let $u, v \in V(T)$ be such that $x \in B(u), y \in B(v)$.

(i) If $w \in V(T)$ lies on the path from $u$ to $v$ in $T$ then $x \leqslant y$ hits $B(w)$.

(ii) If $e=w_{1} w_{2} \in E(T)$ lies on the path from $u$ to $v$ in $T$ then $x \leqslant y$ hits $B\left(w_{1}\right) \cap B\left(w_{2}\right)$.

(iii) If $w_{1}, \ldots, w_{t} \in V(T)$ are $t$ nodes on the path from $u$ to $v$ in $T$ appearing in this order, then there exist $z_{i} \in B\left(w_{i}\right)$ for each $i \in\{1, \ldots, t\}$ such that $x \leqslant z_{1} \leqslant \cdots \leqslant z_{t} \leqslant y$ in $P$.

Proof Suppose that $w$ lies on a path from $u$ to $v$ in $T$. Since $x \leqslant y$ in $P$ there is a path $x=z_{0}, z_{1}, \ldots, z_{k}=y$ in $G$ such that $z_{i}<z_{i+1}$ is a cover relation in $P$ for each $i \in$ $\{0,1, \ldots, k-1\}$. This means that $\bigcup_{0 \leqslant i \leqslant k} T_{z_{i}}$ is a (connected) subtree of $T$ containing $u$ and $v$. Thus, $\bigcup_{0 \leqslant i \leqslant k} T_{z_{i}}$ contains $w$ and therefore there exists $i$ with $z_{i} \in B(w)$. The proof of (ii) is analogous.

We prove (iii) by induction on $t$. For $t=1$ this corresponds to (i), so let us assume $t>1$ and consider the inductive case. By induction there exist $z_{i} \in B\left(w_{i}\right)$ for each $i \in$ $\{1, \ldots, t-1\}$ such that $x \leqslant z_{1} \leqslant \cdots \leqslant z_{t-1} \leqslant y$ in $P$. Applying (i) with relation $z_{t-1} \leqslant y$ and the $w_{t-1}-v$ path, we obtain that $z_{t-1} \leqslant z_{t} \leqslant y$ in $P$ for some $z_{t} \in B\left(w_{t}\right)$. Combining, we obtain $x \leqslant z_{1} \leqslant \cdots \leqslant z_{t} \leqslant y$ in $P$, as desired.

\section{The Proof}

We aim to partition $\mathrm{MM}(P)$ into a constant number of sets, each of which is reversible. This will be realized with the help of a signature tree, which is depicted on Fig. 5. This plane tree $\Psi$, rooted at node $v_{1}$, assigns to each pair $(a, b) \in \mathrm{MM}(P)$ a corresponding leaf of $\Psi$ according to properties of the pair $(a, b)$.

The nodes $v_{1}, \ldots, v_{15}$ of $\Psi$ are enumerated by depth-first and left-to-right search. Each node $v_{i}$ which is distinct from the root and not a leaf has a corresponding function of the form $\alpha_{i}: \operatorname{MM}\left(P, v_{i}\right) \rightarrow \Sigma_{i}$, where $\operatorname{MM}\left(P, v_{i}\right) \subseteq \operatorname{MM}(P)$ and $\Sigma_{i}$ is a finite set, whose size does not depend on $P$. We put $\operatorname{MM}\left(P, v_{1}\right)=\operatorname{MM}(P)$ and the other domains will be defined one by one in this section. To give an example, let us look forward to upcoming subsections where we define $\alpha_{1}$ and $\alpha_{2}$ as follows.

- $\quad \alpha_{1}(a, b) \in \Sigma_{1}=\{$ left, right $\}$ encodes whether $a^{T}$ is to the left or to the right of $b^{T}$ in $T$;

- $\alpha_{2}(a, b) \in \Sigma_{2}=\{$ yes, no $\}$ is the answer to the question "Is there an element $q \in$ $B\left(a^{T} \wedge b^{T}\right)$ with $a \leqslant q$ in $P$ ?".

Furthermore, for each internal node $v_{i}$ with children $v_{i_{1}}, \ldots, v_{i_{l}}$ in $\Psi$, the edges $v_{i} v_{i_{1}}, \ldots, v_{i} v_{i_{l}}$ of $\Psi$ are respectively labeled by subsets $\Sigma\left(v_{i}, v_{i_{1}}\right), \ldots, \Sigma\left(v_{i}, v_{i_{l}}\right)$ of $\Sigma_{i}$ such that $\Sigma_{i}=\Sigma\left(v_{i}, v_{i_{1}}\right) \sqcup \cdots \sqcup \Sigma\left(v_{i}, v_{i_{l}}\right)$, that is, so that the sets $\Sigma\left(v_{i}, v_{i_{j}}\right)$ form a partition of $\Sigma_{i}$. For example,

$$
\begin{aligned}
& \Sigma\left(v_{1}, v_{2}\right)=\{\text { left }, \text { right }\}=\Sigma_{1} ; \\
& \Sigma\left(v_{2}, v_{3}\right)=\{\text { no } ; \\
& \Sigma\left(v_{2}, v_{4}\right)=\{\text { yes }\} .
\end{aligned}
$$

Observe that each internal node $v_{i}$ of $\Psi$ has either one or two children; in particular, if $v_{i}$ has only one child then the corresponding edge is labeled with the full set $\Sigma_{i}$. 


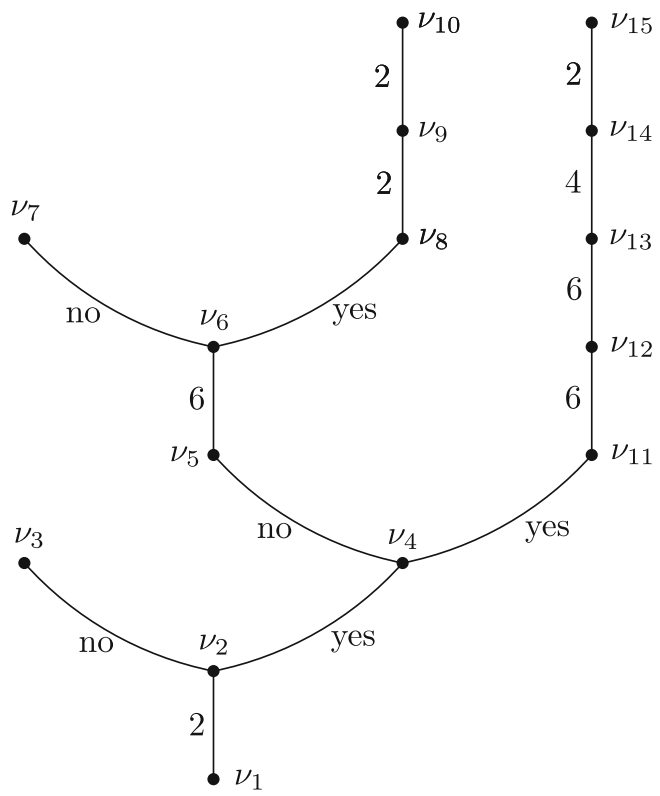

Fig. 5 The signature tree $\Psi$. Each of the three branching nodes $v_{2}, v_{4}$, and $v_{6}$ corresponds to a yes/no question, and the edges towards their children are labeled according to the possible answers. Edges starting from a non-branching node $v_{i}$ to a node $v_{j}$ are labeled with the size of $\Sigma\left(v_{i}, v_{j}\right)$

The reader may wonder why we do not refine the tree $\Psi$ and have an edge out of $v_{i}$ for every possible value in $\Sigma_{i}$. This is because sometimes several values in $\Sigma_{i}$ will correspond to analogous cases in our proofs which can be treated all at once. To give a concrete example, consider $\Sigma\left(v_{1}, v_{2}\right)=\{$ left, right $\}$ : When proving that a set $S$ of min-max pairs is reversible, the case that $a^{T}$ is left of $b^{T}$ for every $(a, b) \in S$ is analogous to the case that $a^{T}$ is right of $b^{T}$ for every $(a, b) \in S$, as one is obtained from the other by exchanging the notion of left and right in $T$ (that is, by replacing the plane tree $T$ by its mirror image). Hence it will be enough to only consider, say, the case where $a^{T}$ is to the left of $b^{T}$ for every $(a, b) \in S$.

Now for an internal node $v_{i}$ of $\Psi$ distinct from the root $(i \neq 1)$, let $v_{1}=v_{i_{1}}, \ldots, v_{i_{l}}=v_{i}$ be the path from the root $v_{1}$ to $v_{i}$ in $\Psi$. Define the signature of $v_{i}$ as the set

$$
\Sigma\left(v_{i}\right)=\Sigma\left(v_{i_{1}}, v_{i_{2}}\right) \times \ldots \times \Sigma\left(v_{i_{l-1}}, v_{i_{l}}\right)
$$

and let

$\operatorname{MM}\left(P, v_{i}\right)=\left\{(a, b) \in \operatorname{MM}(P) \mid\left(\alpha_{i_{1}}(a, b), \ldots, \alpha_{i_{l-1}}(a, b)\right) \in \Sigma\left(v_{i}\right)\right\} ;$

$\operatorname{MM}\left(P, v_{i}, \Sigma\right)=\left\{(a, b) \in \operatorname{MM}(P) \mid\left(\alpha_{i_{1}}(a, b), \ldots, \alpha_{i_{l-1}}(a, b)\right)=\Sigma\right\} \quad$ for $\Sigma \in \Sigma\left(v_{i}\right)$.

Observe that by this definition, for each internal node $v_{i}$ of $\Psi$ with children $v_{i_{1}}, \ldots, v_{i_{l}}$ we get the partition

$$
\operatorname{MM}\left(P, v_{i}\right)=\bigcup_{1 \leqslant j \leqslant l} \operatorname{MM}\left(P, v_{i_{j}}\right)
$$


Therefore, by construction the sets $\operatorname{MM}\left(P, v_{i}\right)$ with $v_{i}$ a leaf of $\Psi$ (so for $v_{3}, v_{7}, v_{10}, v_{15}$ ) form a partition of $\mathrm{MM}(P)$. With a further refinement it follows that

$$
\operatorname{MM}(P)=\bigcup_{v_{i} \text { leaf of } \Psi} \bigcup_{\Sigma \in \Sigma\left(v_{i}\right)} \operatorname{MM}\left(P, v_{i}, \Sigma\right)
$$

and the proof below boils down to showing that $\operatorname{MM}\left(P, v_{i}, \Sigma\right)$ is reversible for each leaf $v_{i}$ of $\Psi$ and each $\Sigma \in \Sigma\left(v_{i}\right)$.

Once this is established we get an upper bound on $\operatorname{dim}^{*}(P)$ just by counting the number of sets in our partition of $\mathrm{MM}(P)$, namely

$$
\begin{aligned}
\operatorname{dim}^{*}(P) \leqslant \sum_{v_{i} \text { leaf of } \Psi}\left|\Sigma\left(v_{i}\right)\right| & =\left|\Sigma\left(v_{3}\right)\right|+\left|\Sigma\left(v_{7}\right)\right|+\left|\Sigma\left(v_{10}\right)\right|+\left|\Sigma\left(v_{15}\right)\right| \\
& =2+2 \cdot 6+2 \cdot 6 \cdot 2 \cdot 2+2 \cdot 6 \cdot 6 \cdot 4 \cdot 2=638 .
\end{aligned}
$$

Note that the calculation for the particular summands follows from (1) and the edge labelings in Fig. 5. Our proof will follow a depth-first, left-to-right search of the signature tree $\Psi$, defining the functions $\alpha_{i}$ one by one in that order, and showing that for each $\Sigma \in$ $\Sigma\left(v_{i}\right)$ the set $\operatorname{MM}\left(P, v_{i}, \Sigma\right)$ is reversible when encountering a leaf $v_{i}$. Hence, the tree $\Psi$ also serves as a road map of the proof. Moreover, for the reader's convenience we included a table collecting all functions $\alpha_{i}$ and their meanings, see Table 1. It is not necessary to read this table now, but it might be helpful while going through the main proof.

Now that the necessary definitions are introduced and the preliminary observations are made, we are about to consider the nodes of the signature tree one by one, stating and proving many technical statements along the way. At this point the reader might legitimately wonder why it all works, that is, what are the basic ideas underlying our approach. While we are unable to offer a general intuition-indeed, this is why we believe that better insights into these posets remain to be obtained-we can at least explain a couple of the strategies we repeatedly apply in our proofs.

A first strategy builds on the fact that when choosing three times an element in a 2element set, some element is bound to be chosen at least twice: As a toy example, suppose that $\left\{\left(a_{i}, b_{i}\right)\right\}_{i=1}^{k}$ is a strict alternating cycle with $k \geqslant 3$ in some subset $I \subseteq \mathrm{MM}(P)$ which we are trying to prove is reversible. Suppose further that we somehow previously established that the $a_{i}^{T}-b_{i+1}^{T}$ path in $T$ includes a specific edge $u v$ of $T$ for at least three distinct indices $i \in\{1, \ldots, k\}$; which indices is not important, so let us say this happens for indices $1,2,3$. Then by Observation 10 the relation $a_{i} \leqslant b_{i+1}$ hits $B(u) \cap B(v)$ for each $i=1,2,3$. Given that $|B(u) \cap B(v)|=2$, this implies that some element $x \in B(u) \cap B(v)$ is hit by two of these relations, that is, we have $a_{i} \leqslant x \leqslant b_{i+1}$ and $a_{j} \leqslant x \leqslant b_{j+1}$ in $P$ for some $i, j \in\{1,2,3\}$ with $i<j$. However, this implies $a_{i} \leqslant x \leqslant b_{j+1}$ in $P$, contradicting the fact that the alternating cycle is strict. (Here we use that $k \geqslant 3$.) Therefore, the alternating cycle $\left\{\left(a_{i}, b_{i}\right)\right\}_{i=1}^{k}$ could not have existed in the first place. More generally, when analyzing certain situations we claim cannot occur, we will typically easily find two relations $c_{1} \leqslant d_{1}$ and $c_{2} \leqslant d_{2}$ in $P$ both hitting $B(u) \cap B(v)$ for some edge $u v$ of $T$, and which are incompatible, in the sense that they cannot hit the same element. The work then goes into pinning down a third relation $c_{3} \leqslant d_{3}$ in $P$ which is incompatible with the first two, and yet hits $B(u) \cap B(v)$. (The fact that $a_{0} \leqslant b$ in $P$ for every $b \in \max (P)$ will often be helpful here.)

A second strategy is to see certain strict alternating cycles as inducing a graph on $\operatorname{MM}(P)$, and then study and exploit properties of said graph. This is natural for strict alternating cycles of length 2 : Any such cycle $\left(a_{1}, b_{1}\right),\left(a_{2}, b_{2}\right)$ can be seen as inducing an edge between vertex $\left(a_{1}, b_{1}\right)$ and vertex $\left(a_{2}, b_{2}\right)$. If we somehow can show that the resulting 
Table 1 Table of functions and their meanings

\begin{tabular}{lll}
\hline Function & Section & Meaning \\
\hline$\phi$ & 2 & Proper 3-coloring of the intersection
\end{tabular}
graph of the subtrees $T_{x}(x \in X)$ of $T$. That is, $\phi(x) \neq \phi(y)$ whenever $V\left(T_{x}\right) \cap V\left(T_{y}\right) \neq \emptyset$.

$\alpha_{1}$

$\alpha_{2}$

$\alpha_{4}$

$\alpha_{5}$

$\alpha_{6}$

$\alpha_{8}$
Assigns 'left' or 'right' to pairs $(a, b)$ depending on whether $a^{T}$ lies to the left or to the right of $b^{T}$ in $T$.

Records the answer to the question: "Is there an element $q \in B\left(u_{a b}\right)$ such that $a \leqslant q$ in $P$ ?"

Records the answer to the question: "Is there an element $q \in B\left(u_{a b}\right) \cap$ $B\left(p_{a b}\right)$ such that $a \leqslant q$ in $P$ ?"

$\alpha_{5}(a, b) \quad=$ $\left(\phi\left(x_{a b}\right), \phi\left(y_{a b}\right), \phi\left(z_{a b}\right)\right), \quad$ where $B\left(u_{a b}\right)=\left\{x_{a b}, y_{a b}, z_{a b}\right\}$ and $x_{a b}, y_{a b}, z_{a b}$ satisfy:

- $a \leqslant x_{a b} \nless b$ in $P$;

- $B\left(u_{a b}\right) \cap B\left(p_{a b}\right)=\left\{y_{a b}, z_{a b}\right\}$

- $a \nless y_{a b} \leqslant b \operatorname{in} P$;

- $a_{0} \leqslant y_{a b}$ in $P$;

- $a \& z_{a b}$ in $P$, and

- $y_{a b} \in B\left(u_{a b}\right) \cap B\left(w_{a b}\right)$.

Records the answer to the question "Is $B\left(u_{a b}\right) \cap B\left(w_{a b}\right)=\left\{x_{a b}, y_{a b}\right\}$ ?". Given $(a, b) \in \operatorname{MM}\left(P, v_{8}\right)$, let $\Sigma \in \Sigma_{8}$ be such that $(a, b) \in$ $\operatorname{MM}\left(P, v_{8}, \Sigma\right)$. Then $\alpha_{8}(a, b)=$ $\psi_{8, \Sigma}(a, b)$, where $\psi_{8, \Sigma}$ is a 2 coloring of the graph $S_{\Sigma}$ of special 2-cycles.

Given $(a, b) \in \operatorname{MM}\left(P, \nu_{9}, \Sigma\right)$ for some $\Sigma \in \Sigma\left(v_{9}\right)$, function $\alpha_{9}$ records the color $\psi_{9, \Sigma}(a, b)$, where $\psi_{9, \Sigma}$ is a coloring of $K_{\Sigma}$.

$\alpha_{11}(a, b) \quad=$ $\left(\phi\left(x_{a b}\right), \phi\left(y_{a b}\right), \phi\left(z_{a b}\right)\right), \quad$ where $B\left(u_{a b}\right)=\left\{x_{a b}, y_{a b}, z_{a b}\right\}$ and $x_{a b}, y_{a b}, z_{a b}$ satisfy:

- $B\left(u_{a b}\right) \cap B\left(\mathrm{p}_{a b}\right)=\left\{x_{a b}, y_{a b}\right\}$;

- $a \leqslant x_{a b} \& y_{a b}$ in $P$, and

- $a \leqslant y_{a b} \leqslant b$ in $P$.

Given $(a, b) \in \operatorname{MM}\left(P, v_{12}\right)$, $\alpha_{12}(a, b)$ records the answers to the questions "Is $a \leqslant z_{a b}$ in $P$ ", "Is $z_{a b} \leqslant b$ in $P$ ?", and "Is $a_{0} \leqslant x_{a b}$ in $P$ ?'. 
Table 1 (continued)

\begin{tabular}{|c|c|c|}
\hline Function & Section & Meaning \\
\hline$\alpha_{13}$ & 3.11 & $\begin{array}{l}\text { Given }(a, b) \in \operatorname{MM}\left(P, v_{13}, \Sigma\right), \\
\text { function } \alpha_{13} \text { records the color of the } \\
\text { pair }(a, b) \text { in the 4-coloring } \psi_{13, \Sigma} \\
\text { of the graph } J_{\Sigma} \text {. The purpose of } \\
\alpha_{13} \text { is to get rid of 2-cycles in } \\
\operatorname{MM}\left(P, v_{13}, \Sigma\right) .\end{array}$ \\
\hline$\alpha_{14}$ & 3.12 & $\begin{array}{l}\text { Given }(a, b) \in \operatorname{MM}\left(P, v_{14}, \Sigma\right) \text {, } \\
\text { function } \alpha_{14} \text { records the color of the } \\
\text { pair }(a, b) \text { in the } 2 \text {-coloring } \psi_{14, \Sigma} \\
\text { of the graph } \hat{K}_{\Sigma} \text {. The purpose of } \\
\alpha_{14} \text { is to get rid of strict alternat- } \\
\text { ing cycles of length at least } 3 \text { in } \\
\operatorname{MM}\left(P, v_{14}, \Sigma\right) .\end{array}$ \\
\hline
\end{tabular}

graph has bounded chromatic number, then we can consider a corresponding coloring of the pairs, and we will know that within a color class there are no strict alternating cycles of length 2 left. Thus, by doing so we 'killed' all such cycles by partitioning the pairs in a constant number of sets. Such a strategy is used twice in the proof, when considering nodes $v_{8}$ and $v_{13}$ of the signature tree $\Psi$. We also use a variant of it tailored to handle certain strict alternating cycles of length at least 3 and involving a directed graph on $\operatorname{MM}(P)$, when considering nodes $\nu_{9}$ and $\nu_{14}$ of $\Psi$.

We now turn to the proof. From now on we will use the following notations for a given pair $(a, b) \in \operatorname{MM}(P)$ : We let $u_{a b}:=a^{T} \wedge b^{T}, p_{a b}:=\mathrm{p}\left(u_{a b}\right)$, and denote by $v_{a b}$ and $w_{a b}$ the neighbors of $u_{a b}$ in $T$ towards $a^{T}$ and $b^{T}$, respectively. Figure 6 illustrates the newly defined nodes.

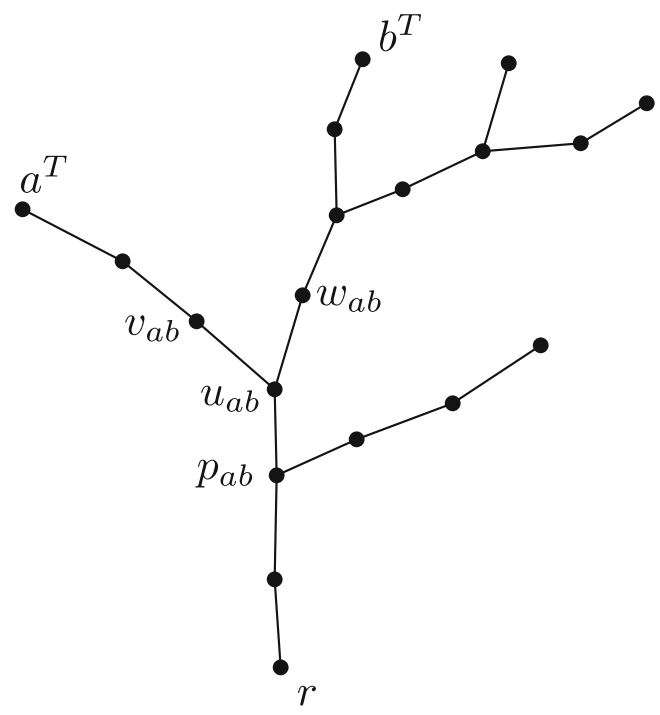

Fig. 6 Pair $(a, b) \in \operatorname{MM}(P)$ with $\alpha_{1}(a, b)=$ left and the corresponding nodes $p_{a b}, u_{a b}, v_{a b}$, and $w_{a b}$ in $T$ 


\subsection{Nodes $v_{1}$ and $v_{2}$ and their respective functions $\alpha_{1}$ and $\alpha_{2}$}

We start with the definition of $\alpha_{1}$, which belongs to the node $v_{1}$ of the signature tree $\Psi$.

For each $(a, b) \in \operatorname{MM}\left(P, \nu_{1}\right)=\operatorname{MM}(P), \alpha_{1}(a, b) \in \boldsymbol{\Sigma}_{1}=\{$ left, right $\}$ encodes whether $a^{T}$ is to the left or to the right of $b^{T}$ in $T$.

Let us proceed with node $v_{2}$ and its function $\alpha_{2}$.

For each $(a, b) \in \operatorname{MM}\left(P, \nu_{2}\right)=\operatorname{MM}(P)$, we let $\alpha_{2}(a, b) \in \boldsymbol{\Sigma}_{2}=\{$ yes, no $\}$ be the answer to the question

"Is there an element $q \in B\left(u_{a b}\right)$ with $a \leqslant q$ in $P$ ?".

Note that it might be the case that all elements of $B\left(u_{a b}\right)$ are incomparable with $a$ (so the answer is "no"), while there is always an element $q \in B\left(u_{a b}\right)$ such that $q \leqslant b$ in $P$ as the comparability $a_{0} \leqslant b$ hits the bag $B\left(u_{a b}\right)$. In the next section we treat all the pairs $(a, b) \in \operatorname{MM}(P)$ with $\alpha_{2}(a, b)=$ no and it turns out that they can easily be reversed.

\subsection{First leaf of $\Psi$ : the node $v_{3}$}

Incomparable pairs of $\operatorname{MM}\left(P, v_{3}\right)$ received one of the two possible signatures in $\Sigma\left(v_{3}\right)=$ $\{($ left, no), (right, no) $\}$. We start by showing that pairs with these signatures are reversible.

Claim $11 \operatorname{MM}\left(P, v_{3}, \Sigma\right)$ is reversible for each $\Sigma \in \Sigma\left(v_{3}\right)$.

Proof Let $\Sigma \in \Sigma\left(v_{3}\right)=\{($ left, no), (right, no) $\}$. We will assume that $\Sigma=$ (left, no), thus $\alpha_{1}(a, b)=$ left for pairs $(a, b) \in \operatorname{MM}\left(P, \nu_{3}, \Sigma\right)$. In the other case it suffices to exchange the notion of left and right in the following argument. (We note that we will start with that assumption in all subsequent proofs, for the same reason.)

Arguing by contradiction, suppose that there is a strict alternating cycle $\left\{\left(a_{i}, b_{i}\right)\right\}_{i=1}^{k}$ in $\operatorname{MM}\left(P, \nu_{3}, \Sigma\right)$. Thus $a_{i} \leqslant b_{i+1}$ in $P$ for all $i$ (cyclically). Let $b_{j}^{T}$ be leftmost in $T$ among all the $b_{i}^{T}$ 's $(i \in\{1, \ldots, k\})$. The node $a_{j}^{T}$ is to the left of $b_{j}^{T}$ (as $\left(a_{j}, b_{j}\right) \in \operatorname{MM}\left(P, \nu_{3}, \Sigma\right)$, so $\alpha_{1}$ $\left(a_{j}, b_{j}\right)=$ left), and thus to the left of all the $b_{i}^{T}$ 's. Hence, the path from $a_{j}^{T}$ to $b_{j+1}^{T}$ in $T$ goes through the node $u_{a_{j} b_{j}}$. By Observation 10, the relation $a_{j} \leqslant b_{j+1}$ in $P$ hits $B\left(u_{a_{j} b_{j}}\right)$, contra$\operatorname{dicting} \alpha_{2}\left(a_{j}, b_{j}\right)=$ no (recall that $\left(a_{j}, b_{j}\right) \in \operatorname{MM}\left(P, v_{3}, \Sigma\right)$, and thus $\alpha_{2}\left(a_{j}, b_{j}\right)=$ no).

As a consequence of Claim 11, all the pairs $(a, b) \in \mathrm{MM}(P)$ being considered in the following satisfy $\alpha_{2}(a, b)=$ yes (see how this fact can be read from the signature tree $\Psi$ ).

\subsection{Nodes $v_{4}$ and $v_{5}$ and their respective functions $\alpha_{4}$ and $\alpha_{5}$}

For all pairs $(a, b)$ in $\operatorname{MM}\left(P, v_{4}\right)$ it holds that there is $q \in B\left(u_{a b}\right)$ such that $a \leqslant q$ in $P$, but it is not clear whether there is such an element being also contained in $B\left(p_{a b}\right)$. It is the purpose of function $\alpha_{4}$ to distinguish the two possible cases at this point.

Given $(a, b) \in \operatorname{MM}\left(P, \nu_{4}\right)$, let $\alpha_{4}(a, b) \in\{$ yes, no $\}$ be the answer to the following question about $(a, b)$ :

"Is there an element $q \in B\left(u_{a b}\right) \cap B\left(p_{a b}\right)$ with $a \leqslant q$ in $P$ ?". 
Before defining the function $\alpha_{5}$ we first show some useful properties of pairs in $\operatorname{MM}\left(P, v_{5}, \Sigma\right)$ for $\Sigma \in \Sigma\left(v_{5}\right)$. Note that these pairs $(a, b)$ satisfy $\alpha_{2}(a, b)=$ yes and $\alpha_{4}(a, b)=$ no.

Claim 12 Let $\Sigma \in \Sigma\left(v_{5}\right)$ and suppose that $\left\{\left(a_{i}, b_{i}\right)\right\}_{i=1}^{k}$ is an alternating cycle in $\operatorname{MM}\left(P, v_{5}, \Sigma\right)$. Let $u_{i}$ denote $u_{a_{i} b_{i}}$, for each $i \in\{1,2, \ldots, k\}$. Then

(i) $u_{i}$ and $u_{i+1}$ are comparable in $T$ for each $i \in\{1,2, \ldots, k\}$, and

(ii) there is an index $j \in\{1,2, \ldots, k\}$ such that $u_{j} \leqslant u_{i}$ in $T$ for each $i \in\{1,2, \ldots, k\}$.

Proof Let $\Sigma \in \Sigma\left(\nu_{5}\right)=\{($ left, yes, no), (right, yes, no) $\}$. Again we may assume $\Sigma=$ (left, yes, no) as the other case is symmetrical. Thus $\alpha_{1}\left(a_{i}, b_{i}\right)=$ left for each $i \in$ $\{1, \ldots, k\}$.

We denote $u_{a_{i} b_{i}}, w_{a_{i} b_{i}}, p_{a_{i} b_{i}}$ by $u_{i}, w_{i}, p_{i}$ respectively, for each $i \in\{1,2, \ldots, k\}$.

To prove the first item observe that since $\alpha_{4}\left(a_{i}, b_{i}\right)=$ no for all pairs $\left(a_{i}, b_{i}\right)$, and since $a_{i} \leqslant b_{i+1}$ in $P$, we have $u_{i}<b_{i+1}^{T}$ in $T$. Indeed, otherwise the path from $a_{i}^{T}$ to $b_{i+1}^{T}$ in $T$ would go through $u_{i}$ and $p_{i}$, and hence $a_{i} \leqslant b_{i+1}$ would hit $B\left(u_{i}\right) \cap B\left(p_{i}\right)$, contradicting $\alpha_{4}\left(a_{i}, b_{i}\right)=$ no. Clearly $u_{i+1}<b_{i+1}^{T}$ in $T$. Therefore, $\left\{u_{i}, u_{i+1}\right\}<b_{i+1}^{T}$ in $T$, which makes $u_{i}$ and $u_{i+1}$ comparable in $T$.

The second item follows immediately from the first item and Observation 8.

Thanks to Claim 12 we know that for every $\Sigma \in \Sigma\left(\nu_{5}\right)$, each alternating cycle in $\operatorname{MM}\left(P, v_{5}, \Sigma\right)$ can be written as $\left\{\left(a_{i}, b_{i}\right)\right\}_{i=1}^{k}$ in such a way that $u_{a_{1} b_{1}} \leqslant u_{a_{i} b_{i}}$ in $T$ for $i \in\{1, \ldots, k\}$. We may further assume that the pair $\left(a_{1}, b_{1}\right)$ is chosen in such a way that

(i) if $\alpha_{1}\left(a_{1}, b_{1}\right)=$ left then $b_{1}^{T}$ is to the right of $b_{i}^{T}$ in $T$ for each $i \in\{2, \ldots, k\}$ satisfying $u_{a_{1} b_{1}}=u_{a_{i} b_{i}}$.

(ii) if $\alpha_{1}\left(a_{1}, b_{1}\right)=$ right then $b_{1}^{T}$ is to the left of $b_{i}^{T}$ in $T$ for each $i \in\{2, \ldots, k\}$ satisfying $u_{a_{1} b_{1}}=u_{a_{i} b_{i}}$.

Note that the pair $\left(a_{1}, b_{1}\right)$ is uniquely defined; we call it the root of the alternating cycle.

Now for each $\Sigma \in \Sigma\left(v_{5}\right)$ and $(a, b) \in \operatorname{MM}\left(P, v_{5}, \Sigma\right)$ we take a closer look at elements in $B\left(u_{a b}\right)$. The bag $B\left(u_{a b}\right)$ consists of three distinct elements; let us denote them $x_{a b}, y_{a b}$, $z_{a b}$. Given that $\alpha_{2}(a, b)=$ yes and $\alpha_{4}(a, b)=$ no, we may assume without loss of generality

$$
\begin{aligned}
& a \leqslant x_{a b} \nless b \text { in } P ; \\
& B\left(u_{a b}\right) \cap B\left(p_{a b}\right)=\left\{y_{a b}, z_{a b}\right\} .
\end{aligned}
$$

Recall that the $u_{a b} w_{a b}$ edge lies on the path from $r$ to $b^{T}$ in $T$. This implies that the relation $a_{0} \leqslant b$ hits $B\left(u_{a b}\right) \cap B\left(w_{a b}\right)$. Clearly, it cannot hit $x_{a b}$, and thus $a_{0} \leqslant b$ hits at least one of $y_{a b}, z_{a b}$. Let us suppose without loss of generality that this is the case for $y_{a b}$. It follows that

$$
\begin{aligned}
& a \nless y_{a b} \leqslant b \text { in } P ; \\
& a_{0} \leqslant y_{a b} \text { in } P ; \\
& a \nless z_{a b} \text { in } P ; \\
& y_{a b} \in B\left(u_{a b}\right) \cap B\left(w_{a b}\right) .
\end{aligned}
$$

With these notations, we define $\alpha_{5}$ in the following way: 
For each $\Sigma \in \boldsymbol{\Sigma}\left(\nu_{5}\right)$ and pair $(a, b) \in \operatorname{MM}\left(P, \nu_{5}, \Sigma\right)$, we let

$$
\alpha_{5}(a, b):=\left(\phi\left(x_{a b}\right), \phi\left(y_{a b}\right), \phi\left(z_{a b}\right)\right) .
$$

(Recall that $\phi(w)$ is the color of the element $w \in X$ in the 3 -coloring $\phi$ of the intersection graph defined by the subtrees $T_{x}(x \in X)$, and that $x_{a b}, y_{a b}, z_{a b}$ have distinct colors.) Hence there are 6 possible answers for $\alpha_{5}(a, b)$. In the following when considering nodes $v_{i}$ of $\Psi$ that are descendants of $v_{5}$, all we will need is that min-max pairs $(a, b) \in \operatorname{MM}\left(P, v_{i}, \Sigma\right)$ have the same value $\alpha_{5}(a, b)$ but the value itself will not be important. This is why $\Psi$ does not branch at $\nu_{5}$.

\subsection{Node $v_{6}$ and its function $\alpha_{6}$}

Before defining the next function $\alpha_{6}$, let us show some useful properties of strict alternating cycles in $\operatorname{MM}\left(P, v_{6}, \Sigma\right)$ for $\Sigma \in \Sigma\left(v_{6}\right)$. These properties will be used not only when considering the second leaf $\nu_{7}$ of $\Psi$ but also later on when considering the third leaf $v_{10}$.

Now, recall that pairs $(a, b) \in \operatorname{MM}\left(P, v_{6}\right)$ satisfy

- $\quad \alpha_{2}(a, b)=$ yes, and hence there is $q \in B\left(u_{a b}\right)$ with $a \leqslant q$ in $P$,

- $\alpha_{4}(a, b)=$ no, and hence there is no $q \in B\left(u_{a b}\right) \cap B\left(p_{a b}\right)$ such that $a \leqslant q$ in $P$,

- the elements of $B\left(u_{a b}\right)$ can be labeled with $x_{a b}, y_{a b}, z_{a b}$ such that (2)-(7) hold.

We need these properties and the mentioned labeling for the following claim.

Claim 13 Let $\Sigma \in \Sigma\left(v_{6}\right)$ and suppose that $\left\{\left(a_{i}, b_{i}\right)\right\}_{i=1}^{k}$ is a strict alternating cycle in $\operatorname{MM}\left(P, v_{6}, \Sigma\right)$ with root $\left(a_{1}, b_{1}\right)$. Let $u_{i}, w_{i}$ denote $u_{a_{i} b_{i}}, w_{a_{i} b_{i}}$ respectively, for each $i \in$ $\{1,2, \ldots, k\}$. Then $u_{1}<w_{1} \leqslant u_{k}<b_{1}^{T}$ in $T$.

Proof We denote $p_{a_{i} b_{i}}, x_{a_{i} b_{i}}, y_{a_{i} b_{i}}, z_{a_{i} b_{i}}$ by $p_{i}, x_{i}, y_{i}, z_{i}$ respectively, for each $i \in$ $\{1,2, \ldots, k\}$. We assume that $\alpha_{1}(a, b)=$ left for each $(a, b) \in \mathrm{MM}\left(P, v_{6}, \Sigma\right)$. In particular, $\alpha_{1}\left(a_{i}, b_{i}\right)=$ left for each $i \in\{1,2, \ldots, k\}$.

The path from $a_{k}^{T}$ to $b_{1}^{T}$ in $T$ cannot go through the edge $u_{k} p_{k}$, since otherwise by Observation 10 the relation $a_{k} \leqslant b_{1}$ would hit $B\left(u_{k}\right) \cap B\left(p_{k}\right)$ which contradicts the fact that $\alpha_{4}\left(a_{k}, b_{k}\right)=$ no. This implies $u_{k}<b_{1}^{T}$ in $T$.

Next we prove that $u_{1} \neq u_{k}$. Suppose to the contrary that $u_{1}=u_{k}$. Then $u_{k}$ lies on the paths from $a_{k}^{T}$ to $r$ and from $b_{1}^{T}$ to $r$ in $T$, implying that $a_{k}^{T} \wedge b_{1}^{T} \geqslant u_{k}$ in $T$.

If $a_{k}^{T} \wedge b_{1}^{T}=u_{k}$ then the path from $a_{k}^{T}$ to $b_{1}^{T}$ in $T$ goes through $u_{k}$. Thus, the relation $a_{k} \leqslant b_{1}$ hits $B\left(u_{k}\right)=\left\{x_{k}, y_{k}, z_{k}\right\}$ and hence $a_{k} \leqslant x_{k} \leqslant b_{1}$ in $P\left(\right.$ as $a_{k} \nless y_{k}$ and $a_{k} \nless z_{k}$ in $P)$. Since $B\left(u_{k}\right)=B\left(u_{1}\right)$ and $\alpha_{5}\left(a_{1}, b_{1}\right)=\alpha_{5}\left(a_{k}, b_{k}\right)$ implying $\phi\left(x_{k}\right)=\phi\left(x_{1}\right)$, we get $x_{k}=x_{1}$. Now $a_{1} \leqslant x_{1}=x_{k} \leqslant b_{1}$ in $P$ gives a contradiction.

If $a_{k}^{T} \wedge b_{1}^{T}>u_{k}$ in $T$ then it follows that $v_{k} \leqslant b_{1}^{T}$ in $T$. By Observation 9(ii) we conclude that $b_{1}^{T}$ is left of $b_{k}^{T}$ in $T$. Since $u_{k}=u_{1}$, this contradicts the fact that $\left(a_{1}, b_{1}\right)$ is the root of $\left\{\left(a_{i}, b_{i}\right)\right\}_{i=1}^{k}$.

Therefore, $u_{1} \neq u_{k}$ as claimed, and $u_{1}<u_{k}<b_{1}^{T}$ in $T$. Given the definition of $w_{1}$ and the fact that $u_{k}<b_{1}^{T}$ in $T$, we deduce $u_{1}<w_{1} \leqslant u_{k}<b_{1}^{T}$ in $T$.

Claim 14 Let $\Sigma \in \Sigma\left(v_{6}\right)$ and suppose that $\left\{\left(a_{i}, b_{i}\right)\right\}_{i=1}^{k}$ is a strict alternating cycle in $\operatorname{MM}\left(P, v_{6}, \Sigma\right)$ with root $\left(a_{1}, b_{1}\right)$. Let $u_{i}, w_{i}$ denote $u_{a_{i} b_{i}}, w_{a_{i} b_{i}}$ respectively, for each $i \in$ $\{1,2, \ldots, k\}$. Then $u_{1}<w_{1} \leqslant u_{i}$ in $T$ for each $i \in\{2, \ldots, k\}$. 
Proof We denote $v_{a_{i} b_{i}}, p_{a_{i} b_{i}}, x_{a_{i} b_{i}}, y_{a_{i} b_{i}}, z_{a_{i} b_{i}}$ by $v_{i}, p_{i}, x_{i}, y_{i}, z_{i}$ respectively, for each $i \in$ $\{1,2, \ldots, k\}$. We assume that $\alpha_{1}(a, b)=$ left for each $(a, b) \in \operatorname{MM}\left(P, v_{6}, \Sigma\right)$. In particular, $\alpha_{1}\left(a_{i}, b_{i}\right)=$ left for each $i \in\{1,2, \ldots, k\}$.

By Claim 13 we have $w_{1} \leqslant u_{k}$ in $T$. Arguing by contradiction, suppose that $w_{1} \nless u_{i}$ for some $i \in\{2, \ldots, k-1\}$, and let $i$ be the largest such index. Thus, $w_{1} \leqslant u_{i+1}$ in $T$. Note also that in this case we must have $k \geqslant 3$.

Since $u_{i}$ and $u_{i+1}$ are comparable in $T$ (by Claim 12) and $u_{1}$ is minimal in $T$ among all the $u_{i}$ 's, we obtain $u_{1}=u_{i}<w_{1} \leqslant u_{i+1}$ in $T$.

Observe that $u_{i} \leqslant a_{i}^{T} \wedge b_{i+1}^{T}$ in $T$, as $u_{i}<\left\{a_{i}^{T}, b_{i+1}^{T}\right\}$ in $T$. If $u_{i}=a_{i}^{T} \wedge b_{i+1}^{T}$ then the path from $a_{i}^{T}$ to $b_{i+1}^{T}$ in $T$ goes through $u_{i}$. Thus, the relation $a_{i} \leqslant b_{i+1}$ hits $B\left(u_{i}\right)=\left\{x_{i}, y_{i}, z_{i}\right\}$, and it follows that $a_{i} \leqslant x_{i} \leqslant b_{i+1}$ in $P$ (as $a_{i} \nless y_{i}$ and $a_{i} \nless z_{i}$ ). Since $B\left(u_{i}\right)=B\left(u_{1}\right)$ and $\phi\left(x_{i}\right)=\phi\left(x_{1}\right)$ (because $\left.\alpha_{5}\left(a_{1}, b_{1}\right)=\alpha_{5}\left(a_{i}, b_{i}\right)\right)$, we deduce that $x_{i}=x_{1}$. But then $a_{1} \leqslant x_{1}=x_{i} \leqslant b_{i+1}$ in $P$, which is a contradiction. (Recall that $i \geqslant 2$.)

If $u_{1}=u_{i}<a_{i}^{T} \wedge b_{i+1}^{T}$ in $T$ then we must have $w_{1} \leqslant a_{i}^{T} \wedge b_{i+1}^{T}$ in $T$, since $a_{i}^{T} \wedge b_{i+1}^{T}$ has to be an internal node of the path from $u_{i}$ to $b_{i+1}^{T}$ in $T$ and since $w_{1}$ is the neighbor of $u_{i}$ on that path (as $u_{i}<w_{1} \leqslant u_{i+1}<b_{i+1}^{T}$ in $T$ ). In particular, this implies $u_{i}<w_{1}<a_{i}^{T}$ in $T$, and it follows that $v_{i}=w_{1}$. As $a_{i}^{T}$ is left of $b_{i}^{T}$ in $T$, and since $v_{i}=w_{1}<b_{1}^{T}$ in $T$, by Observation 9(ii) we obtain that $b_{1}^{T}$ is left of $b_{i}^{T}$, which contradicts the fact that $\left(a_{1}, b_{1}\right)$ is the root of $\left\{\left(a_{i}, b_{i}\right)\right\}_{i=1}^{k}$. This completes the proof.

Now let us define the function $\alpha_{6}$.

We set $\alpha_{6}(a, b)$ to be the answer to the following question:

"Is $B\left(u_{a b}\right) \cap B\left(w_{a b}\right)=\left\{x_{a b}, y_{a b}\right\}$ ?".

Note that $y_{a b}$ always belongs to the intersection, and hence $\alpha_{6}$ tells us whether $x_{a b}$ or $z_{a b}$ is the other element in $B\left(u_{a b}\right) \cap B\left(w_{a b}\right)$. If the answer to this question is "no", then our signature tree leads us to the second leaf of $\Psi$, leaf $\nu_{7}$.

\subsection{Second leaf of $\Psi$ : the node $v_{7}$}

In this section we show that incomparable pairs in $\operatorname{MM}\left(P, v_{7}, \Sigma\right)$ are reversible for each $\Sigma \in \Sigma\left(v_{7}\right)$. Recall that $\operatorname{MM}\left(P, v_{7}\right)$ is a subset of $\operatorname{MM}\left(P, v_{6}\right)$, allowing us to use the observations and claims from the previous section. Moreover, for every pair $(a, b) \in \operatorname{MM}\left(P, v_{7}\right)$ we have that $\alpha_{6}(a, b)=$ no, implying that $B\left(u_{a b}\right) \cap B\left(w_{a b}\right)=\left\{y_{a b}, z_{a b}\right\}$.

Claim $15 \operatorname{MM}\left(P, v_{7}, \Sigma\right)$ is reversible for each $\Sigma \in \Sigma\left(v_{7}\right)$.

Proof Let $\Sigma \in \Sigma\left(v_{7}\right)$. We assume that $\alpha_{1}(a, b)=$ left for each $(a, b) \in \operatorname{MM}\left(P, v_{7}, \Sigma\right)$. In particular, $\alpha_{1}\left(a_{i}, b_{i}\right)=$ left for each $i \in\{1,2, \ldots, k\}$.

Arguing by contradiction, suppose that there is a strict alternating cycle $\left\{\left(a_{i}, b_{i}\right)\right\}_{i=1}^{k}$ in $\operatorname{MM}\left(P, v_{7}, \Sigma\right)$ with root $\left(a_{1}, b_{1}\right)$. We have $u_{1}<w_{1} \leqslant u_{2}$ in $T$ by Claim 14, and in particular $a_{1}^{T} \wedge b_{2}^{T}=u_{1}$. Thus, the path from $a_{1}^{T}$ to $b_{2}^{T}$ in $T$ includes the edge $u_{1} w_{1}$. Hence, the relation $a_{1} \leqslant b_{2}$ hits $B\left(u_{1}\right) \cap B\left(w_{1}\right) \subseteq\left\{x_{1}, y_{1}, z_{1}\right\}$. Since $a_{1} \leqslant x_{1}$ and $a_{1} \|\left\{y_{1}, z_{1}\right\}$ in $P$ we obtain $x_{1} \in B\left(u_{1}\right) \cap B\left(w_{1}\right)$. Recalling that we also have $y_{1} \in B\left(u_{1}\right) \cap B\left(w_{1}\right)$, it follows that $B\left(u_{1}\right) \cap B\left(w_{1}\right)=\left\{x_{1}, y_{1}\right\}$, contradicting $\alpha_{6}\left(a_{1}, b_{1}\right)=$ no. 


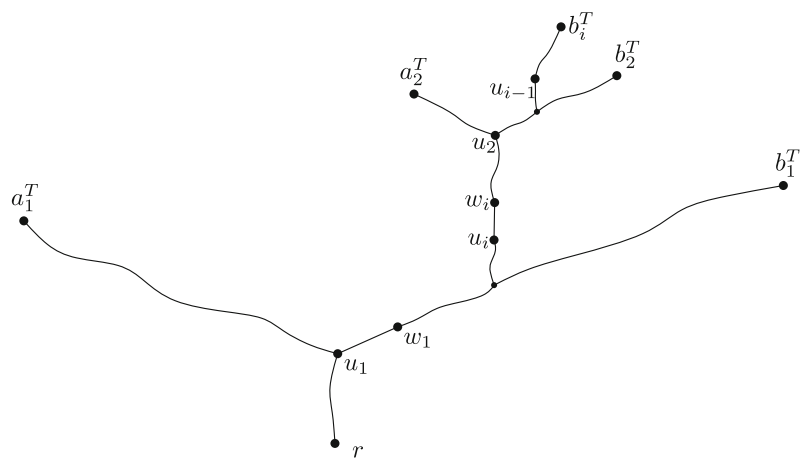

Fig. 7 A possible situation in the proof of Claim 16

\subsection{Node $v_{8}$ and its function $\alpha_{8}$}

Before we study strict alternating cycles in $\operatorname{MM}\left(P, v_{8}\right)$, let us recall some useful properties of incomparable pairs in $\operatorname{MM}\left(P, v_{8}\right)$. Each pair $(a, b) \in \operatorname{MM}\left(P, v_{8}\right)$ satisfies

- $\quad \alpha_{4}(a, b)=$ no, and hence there is no $q \in B\left(u_{a b}\right) \cap B\left(p_{a b}\right)$ such that $a \leqslant q$ in $P$,

- the elements of $B\left(u_{a b}\right)$ can be labeled with $x_{a b}, y_{a b}, z_{a b}$ such that (2)-(7) hold,

- $\quad \alpha_{6}(a, b)=$ yes, and hence $B\left(u_{a b}\right) \cap B\left(w_{a b}\right)=\left\{x_{a b}, y_{a b}\right\}$.

We proceed with an observation about $\operatorname{MM}\left(P, v_{8}, \Sigma\right)$ for fixed $\Sigma \in \Sigma\left(v_{8}\right)$.

Claim 16 Let $\Sigma \in \Sigma\left(v_{8}\right)$ and suppose that $\left\{\left(a_{i}, b_{i}\right)\right\}_{i=1}^{k}$ is a strict alternating cycle in $\operatorname{MM}\left(P, v_{8}, \Sigma\right)$ with root $\left(a_{1}, b_{1}\right)$. Let $u_{i}$ denote $u_{a_{i} b_{i}}$ for each $i \in\{1,2, \ldots, k\}$. Then $u_{1}<$ $w_{1} \leqslant u_{2}<b_{1}^{T}$ in $T$.

Proof We denote $w_{a_{i} b_{i}}, p_{a_{i} b_{i}}, x_{a_{i} b_{i}}, y_{a_{i} b_{i}}, z_{a_{i} b_{i}}$ by $w_{i}, p_{i}, x_{i}, y_{i}, z_{i}$ respectively, for each $i \in\{1,2, \ldots, k\}$. We assume that $\alpha_{1}(a, b)=$ left for each $(a, b) \in \operatorname{MM}\left(P, v_{8}, \Sigma\right)$. In particular, $\alpha_{1}\left(a_{i}, b_{i}\right)=$ left for each $i \in\{1,2, \ldots, k\}$.

If $k=2$ then the claim follows from Claim 13. So we assume $k \geqslant 3$ from now on. By Claim 14 we already know $u_{1}<w_{1} \leqslant u_{2}$ in $T$, and thus it remains to show $u_{2}<b_{1}^{T}$ in $T$.

Arguing by contradiction suppose that $u_{2} \nless b_{1}^{T}$ in $T$. Let $i \in\{3, \ldots, k\}$ be smallest such that $u_{i} \ngtr u_{2}$ in $T$. There is such an index since $u_{k}<b_{1}^{T}$ in $T$ by Claim 13, and thus $u_{k} \ngtr u_{2}$ in $T$. See Fig. 7 for an illustration of this and upcoming arguments.

By our choice of $i$, we have $u_{1}<u_{2} \leqslant u_{i-1}$ in $T$. Note that $a_{i-1} \leqslant b_{i}$ in $P$, which combined with $\alpha_{4}\left(a_{i-1}, b_{i-1}\right)=$ no yields $u_{i-1}<b_{i}^{T}$ in $T$. Hence $u_{2} \leqslant u_{i-1}<b_{i}^{T}$ in $T$.

Since $u_{2}<b_{i}^{T}$ and $u_{i}<b_{i}^{T}$ in $T$, the two nodes $u_{2}$ and $u_{i}$ are comparable in $T$, and thus $u_{i}<u_{2}$ since $u_{i} \ngtr u_{2}$ in $T$. Combining this with $u_{2}<b_{i}^{T}$ in $T$ we further deduce that $w_{i} \leqslant u_{2}$ in $T$. On the other hand, $w_{1} \leqslant u_{i}$ in $T$ by Claim 14. To summarize, we have $u_{1}<w_{1} \leqslant u_{i}<w_{i} \leqslant u_{2}<b_{i}^{T}$ in $T$.

Now consider the path from $a_{1}^{T}$ to $b_{2}^{T}$ in $T$. Since $w_{1}<u_{2} \leqslant b_{2}^{T}$ and $w_{1} \nless a_{1}^{T}$ in $T$, this path goes through $u_{1}$, and thus includes the edge $u_{i} w_{i}$. Hence the relation $a_{1} \leqslant b_{2}$ hits the set $B\left(u_{i}\right) \cap B\left(w_{i}\right)$, the latter being equal to $\left\{x_{i}, y_{i}\right\}$ since $\alpha_{6}\left(a_{i}, b_{i}\right)=$ yes. Therefore, $a_{1} \leqslant x_{i} \leqslant b_{2}$ or $a_{1} \leqslant y_{i} \leqslant b_{2}$ in $P$. But this implies $a_{i} \leqslant x_{i} \leqslant b_{2}$ or $a_{1} \leqslant y_{i} \leqslant b_{i}$ 
in $P$, a contradiction in both cases to the properties of a strict alternating cycle (recall that $i \in\{3, \ldots, k\})$.

Given $\Sigma \in \Sigma\left(v_{8}\right)$, we say that pairs $(a, b),\left(a^{\prime}, b^{\prime}\right) \in \operatorname{MM}\left(P, v_{8}, \Sigma\right)$ form a special 2-cycle if, exchanging $(a, b)$ and $\left(a^{\prime}, b^{\prime}\right)$ if necessary, we have

(i) $a \leqslant b^{\prime}$ and $a^{\prime} \leqslant b$ in $P$, and

(ii) $u_{a b}<w_{a b} \leqslant u_{a^{\prime} b^{\prime}}<w_{a^{\prime} b^{\prime}}<b^{T}$ in $T$.

The first requirement is simply that $(a, b),\left(a^{\prime}, b^{\prime}\right)$ is a (strict) alternating cycle. (Note that every alternating cycle of length 2 is strict.) As a consequence of this and Claim 16, we know that the first three inequalities of the second requirement are satisfied. So the question here is whether also $w_{a^{\prime} b^{\prime}}<b^{T}$ holds in $T$. An implication of the second requirement is that the paths from $a^{T}$ to $b^{\prime T}$ and from $a^{\prime T}$ to $b^{T}$ in $T$ both go through the edge $u_{a^{\prime} b^{\prime}} w_{a^{\prime} b^{\prime}}$ of $T$. Note also that the pair $(a, b)$ is the root this special 2-cycle.

Let $S_{\Sigma}$ be the graph with vertex set $\operatorname{MM}\left(P, v_{8}, \Sigma\right)$ where distinct pairs $(a, b),\left(a^{\prime}, b^{\prime}\right) \in$ $\operatorname{MM}\left(P, v_{8}, \Sigma\right)$ are adjacent if and only if they form a special 2-cycle.

Claim 17 The graph $S_{\Sigma}$ is bipartite for each $\Sigma \in \Sigma\left(v_{8}\right)$.

Proof Arguing by contradiction, suppose that there is an odd cycle $C=\left\{\left(a_{i}, b_{i}\right)\right\}_{i=1}^{k}$ in $S_{\Sigma}$ for some $\Sigma \in \Sigma\left(v_{8}\right)$. We may assume that $C$ is induced. Let $u_{i}:=u_{a_{i} b_{i}}, w_{i}:=w_{a_{i} b_{i}}$, $x_{i}:=x_{a_{i} b_{i}}$ and $y_{i}:=y_{a_{i} b_{i}}$ for each $i \in\{1, \ldots, k\}$.

First we consider the case $k=3$. Since $u_{1}, u_{2}, u_{3}$ are pairwise comparable in $T$, we may assume that $u_{1}<u_{3}<u_{2}$ in $T$ (recall that consecutive $u_{i}$ 's are distinct by property (ii) of special 2-cycles). By the definition of special 2-cycles, we then obtain $u_{2}<w_{2}<$ $\left\{b_{1}^{T}, b_{2}^{T}, b_{3}^{T}\right\}$ in $T$. Thus the paths from $a_{1}^{T}$ to $b_{2}^{T}$, from $a_{2}^{T}$ to $b_{3}^{T}$, and from $a_{3}^{T}$ to $b_{1}^{T}$ in $T$ all go through the edge $u_{2} w_{2}$. This implies that two relations must hit the same element and therefore there is $i \in\{1,2,3\}$ and $q \in B\left(u_{2}\right) \cap B\left(w_{2}\right)$ such that $a_{i} \leqslant q \leqslant b_{i+1}$ and $a_{i+1} \leqslant q \leqslant b_{i+2}$ in $P$ (indices are taken cyclically). However, this gives $a_{i+1} \leqslant b_{i+1}$ in $P$, a contradiction.

Next consider the case $k \geqslant 5$. We will show that $C$ has a chord, contradicting the fact that $C$ is induced. (We remark that the parity of $k$ will not be used here, only that $k \geqslant 5$.)

We may suppose that $u_{2}$ is maximal in $T$ among all the $u_{i}$ 's. We may also assume without loss of generality $u_{1} \leqslant u_{3}<u_{2}$ in $T$. (Recall that by property (ii) of special 2-cycles $u_{i}$ and $u_{i+1}$ are comparable in $T$ and distinct for each $i \in\{1, \ldots, k\}$.) Let $i, j$ be such that $\{i, j\}=\{3,4\}$ and $u_{j}<u_{i}$ in $T$. (Note that $u_{j} \neq u_{i}$ since $\left(a_{j}, b_{j}\right),\left(a_{i}, b_{i}\right)$ form a special 2-cycle.) We claim that

$$
w_{j}<w_{i} \leqslant w_{2} \text { and } w_{1} \leqslant w_{i}
$$

in $T$.

The inequality $w_{j}<w_{i}$ follows from the fact that $\left(a_{j}, b_{j}\right)$ and $\left(a_{i}, b_{i}\right)$ form a special 2-cycle, and thus in particular $u_{j}<w_{j} \leqslant u_{i}<w_{i}$ in $T$. For the inequality $w_{i} \leqslant w_{2}$ we do a case distinction. If $i=3$ then $\left(a_{2}, b_{2}\right)$ and $\left(a_{i}, b_{i}\right)$ form a special 2-cycle with $u_{i}<w_{i} \leqslant u_{2}<w_{2}$ in $T$. If $i=4$ then $\left(a_{2}, b_{2}\right),\left(a_{3}, b_{3}\right)$ as well as $\left(a_{3}, b_{3}\right),\left(a_{i}, b_{i}\right)$ form special 2-cycles. In particular, $\left\{w_{2}, w_{i}\right\}<b_{3}^{T}$ in $T$. This makes $w_{2}$ and $w_{i}$ comparable in $T$ and by the choice of $u_{2}$ we have $w_{i} \leqslant w_{2}$, as desired. Besides this, we also have $w_{1}<w_{2}$ in $T$ (as $\left(a_{1}, b_{1}\right),\left(a_{2}, b_{2}\right)$ form a special 2-cycle), which makes $w_{1}$ and $w_{i}$ comparable in $T$. Since we have $u_{1} \leqslant u_{i}$ by our choice of $i$ and by the assumption that $u_{1} \leqslant u_{3}$ in $T$, it follows that $w_{1} \leqslant w_{i}$ in $T$ and (8) is proven. 
Now, we are going to argue that the $a_{1}^{T}-b_{2}^{T}$ path, the $a_{0}-b_{1}^{T}$ path, the $a_{j}^{T}-b_{i}^{T}$ path, and the $a_{i}^{T}-b_{j}^{T}$ path all go through the edge $u_{i} w_{i}$ in $T$.

For the $a_{1}^{T}-b_{2}^{T}$ path note that $u_{1}<w_{1} \leqslant w_{i} \leqslant w_{2}<b_{2}^{T}$ in $T$. This implies that this path has to go first from $a_{1}^{T}$ down to $u_{1}$ and then pursue with the $u_{1}-b_{2}^{T}$ path, which includes $w_{i}$ by the previous inequalities, and thus also its parent $u_{i}$ (since $u_{1}<w_{i}$ in $T$ ). Hence, it includes the edge $u_{i} w_{i}$ of $T$.

For the $a_{0}-b_{1}^{T}$ path it suffices to observe that $r<u_{i}<w_{i} \leqslant w_{2}<b_{1}^{T}$ in $T$. Similarly, for the $a_{j}^{T}-b_{i}^{T}$ path, notice that $u_{j}<w_{j} \leqslant u_{i}<w_{i}<b_{i}^{T}$ in $T$. Finally, for the $a_{i}^{T}-b_{j}^{T}$ path, observe that $u_{i}<w_{i}<b_{j}^{T}$ in $T$.

Using Observation 10, it follows that the relations $a_{1} \leqslant b_{2}, a_{0} \leqslant b_{1}, a_{j} \leqslant b_{i}$ and $a_{i} \leqslant b_{j}$ in $P$ all hit $B\left(u_{i}\right) \cap B\left(w_{i}\right)=\left\{x_{i}, y_{i}\right\}$. Clearly,

$$
a_{j} \leqslant y_{i} \leqslant b_{i} \quad \text { and } \quad a_{i} \leqslant x_{i} \leqslant b_{j}
$$

in $P$.

Now, in $P$ we either have $a_{0} \leqslant x_{i} \leqslant b_{1}$ and $a_{1} \leqslant y_{i} \leqslant b_{2}$, or $a_{0} \leqslant y_{i} \leqslant b_{1}$ and $a_{1} \leqslant x_{i} \leqslant b_{2}$. This implies $a_{i} \leqslant b_{1}$ and $a_{1} \leqslant b_{i}$, or $a_{j} \leqslant b_{1}$ and $a_{1} \leqslant b_{j}$.

In the first case, $\left(a_{1}, b_{1}\right),\left(a_{i}, b_{i}\right)$ is an alternating cycle of length 2 (and thus is strict). Recall that $w_{2}<b_{1}^{T}$ in $T$, which together with (8) yields $w_{1} \leqslant w_{i}<b_{1}^{T}$ in $T$. Furthermore, applying Claim 14 on $\left(a_{1}, b_{1}\right),\left(a_{i}, b_{i}\right)$ we obtain $u_{1} \neq u_{i}$, implying $w_{1} \neq w_{i}$, and hence $w_{1}<w_{i}$ in $T$. It follows that $u_{1}<w_{1} \leqslant u_{i}<w_{i}<b_{1}^{T}$ in $T$, which shows that $\left(a_{1}, b_{1}\right),\left(a_{i}, b_{i}\right)$ is a special 2 -cycle. This gives us a chord of the cycle $C$, a contradiction.

In the second case, $\left(a_{1}, b_{1}\right),\left(a_{j}, b_{j}\right)$ is a (strict) alternating cycle. Moreover, $w_{1} \leqslant w_{i}$ and $w_{j} \leqslant w_{i}$ in $T$ (see (8)), which makes $w_{1}$ and $w_{j}$ comparable in $T$. Again by Claim 14 we get $w_{1} \neq w_{j}$. If $w_{1}<w_{j}$ then it also holds that $u_{1}<w_{1} \leqslant u_{j}<w_{j}<b_{1}^{T}$ in $T$ (as $w_{j} \leqslant w_{2}<b_{1}^{T}$ ). If $w_{j}<w_{1}$ then it follows that $u_{j}<w_{j} \leqslant u_{1}<w_{1}<b_{j}^{T}$ in $T$ (as $\left.w_{1} \leqslant w_{i}<b_{j}^{T}\right)$. Thus in both cases $\left(a_{1}, b_{1}\right),\left(a_{j}, b_{j}\right)$ is a special 2-cycle and a chord in $C$, a contradiction. This completes the proof.

Using Claim 17, for each $\Sigma \in \Sigma\left(v_{8}\right)$ let $\psi_{8, \Sigma}: \operatorname{MM}\left(P, v_{8}, \Sigma\right) \rightarrow\{1,2\}$ be a (proper) 2-coloring of $S_{\Sigma}$. The function $\alpha_{8}$ then simply records the color of a pair in this coloring:

For each $\Sigma \in \boldsymbol{\Sigma}\left(\nu_{8}\right)$ and each pair $(a, b) \in \operatorname{MM}\left(P, \nu_{8}, \Sigma\right)$, we let

$$
\alpha_{8}(a, b):=\psi_{8, \Sigma}(a, b) \text {. }
$$

\subsection{Node $v_{9}$ and its function $\alpha_{9}$}

By the definition of $\alpha_{8}$ there is no special 2-cycle in $\operatorname{MM}\left(P, v_{9}, \Sigma\right)$, for every $\Sigma \in \Sigma\left(v_{9}\right)$. This will be used in the definition of the function $\alpha_{9}$.

In order to define $\alpha_{9}$ we first need to introduce an auxiliary directed graph. For each $\Sigma \in \Sigma\left(v_{9}\right)$, let $K_{\Sigma}$ be the directed graph with vertex set $\operatorname{MM}\left(P, v_{9}, \Sigma\right)$ where for any two distinct pairs $\left(a_{1}, b_{1}\right),\left(a_{2}, b_{2}\right) \in \operatorname{MM}\left(P, v_{9}, \Sigma\right)$ there is an arc from $\left(a_{1}, b_{1}\right)$ to $\left(a_{2}, b_{2}\right)$ in $K_{\Sigma}$ if there exists a strict alternating cycle $\left\{\left(a_{i}^{\prime}, b_{i}^{\prime}\right)\right\}_{i=1}^{k}$ in $\operatorname{MM}\left(P, v_{9}, \Sigma\right)$ with root $\left(a_{1}^{\prime}, b_{1}^{\prime}\right)$ such that $\left(a_{1}^{\prime}, b_{1}^{\prime}\right)=\left(a_{1}, b_{1}\right)$ and $\left(a_{2}^{\prime}, b_{2}^{\prime}\right)=\left(a_{2}, b_{2}\right)$. In that case we say that the arc $f$ is induced by the strict alternating cycle $\left\{\left(a_{i}^{\prime}, b_{i}^{\prime}\right)\right\}_{i=1}^{k}$. (Note that there could possibly be different strict alternating cycles inducing the same arc $f$.) 
Claim 18 Let $\Sigma \in \Sigma\left(\nu_{9}\right)$. Then for each arc $\left(\left(a_{1}, b_{1}\right),\left(a_{2}, b_{2}\right)\right)$ in $K_{\Sigma}$ we have

(i) $x_{1} \leqslant y_{2}$, and

(ii) $y_{1} \leqslant z_{2} \leqslant b_{1}$

in $P$, where $x_{i}:=x_{a_{i} b_{i}}, y_{i}:=y_{a_{i} b_{i}}$, and $z_{i}:=z_{a_{i} b_{i}}$ for $i=1,2$.

Proof Let $u_{i}:=u_{a_{i} b_{i}}, p_{i}:=p_{a_{i} b_{i}}$, and $w_{i}:=w_{a_{i} b_{i}}$ for $i=1,2$. By the definition of an arc in $K_{\Sigma}$ and by Claim 16 it holds that $u_{1}<w_{1} \leqslant u_{2}<b_{1}^{T}$ in $T$. Thus, the path from $a_{1}^{T}$ to $b_{2}^{T}$ in $T$ goes through $u_{1}, w_{1}, u_{2}$ and $w_{2}$. Hence, the relation $a_{1} \leqslant b_{2}$ (which exists by the definition of an arc in $K_{\Sigma}$ ) hits $B\left(u_{1}\right) \cap B\left(w_{1}\right)=\left\{x_{1}, y_{1}\right\}$ and $B\left(u_{2}\right) \cap B\left(w_{2}\right)=\left\{x_{2}, y_{2}\right\}$. It cannot hit $y_{1}$ (otherwise $a_{1} \leqslant y_{1} \leqslant b_{1}$ in $P$ ) nor $x_{2}$ (otherwise $a_{2} \leqslant x_{2} \leqslant b_{2}$ in $P$ ). It follows that $a_{1} \leqslant x_{1} \leqslant y_{2} \leqslant b_{2}$ in $P$ by Observation 10 , and 18 is proven.

For (ii) observe that the relation $a_{0} \leqslant b_{1}$ hits $\left\{x_{1}, y_{1}\right\}=B\left(u_{1}\right) \cap B\left(w_{1}\right)$ and $\left\{y_{2}, z_{2}\right\}=$ $B\left(p_{2}\right) \cap B\left(u_{2}\right)$. It cannot hit $x_{1}$ nor $y_{2}$ since otherwise $a_{1} \leqslant b_{1}$ in $P$ as we have $a_{1} \leqslant x_{1} \leqslant y_{2}$ in $P$. Hence we obtain $a_{0} \leqslant y_{1} \leqslant z_{2} \leqslant b_{1}$ in $P$ by Observation 10 .

Let us remark that it is possible to strengthen the statement of Claim 18. In fact, strict inequalities $x_{1}<y_{2}$ and $y_{1}<z_{2}$ hold in (i) and (ii), respectively. Indeed, since $\phi$ is a proper coloring and $\alpha_{5}\left(a_{1}, b_{1}\right)=\alpha_{5}\left(a_{2}, b_{2}\right)$, we have $\phi\left(x_{1}\right)=\phi\left(x_{2}\right) \neq \phi\left(y_{2}\right)$ and $\phi\left(y_{1}\right)=\phi\left(y_{2}\right) \neq \phi\left(z_{2}\right)$, implying that $x_{1} \neq y_{2}$ and $y_{1} \neq z_{2}$. For our purposes the nonstrict inequalities of Claim 18 (and also in forthcoming claims) suffice. As we do not want give more arguments than needed, we will not always prove the strongest possible statement in the following.

Claim 19 Let $\Sigma \in \Sigma\left(v_{9}\right)$. Suppose that $\left(a_{1}, b_{1}\right),\left(a_{2}, b_{2}\right),\left(a_{3}, b_{3}\right) \in \operatorname{MM}\left(P, v_{9}, \Sigma\right)$ are three distinct pairs such that $\left(\left(a_{1}, b_{1}\right),\left(a_{2}, b_{2}\right)\right)$ is an arc in $K_{\Sigma}$ and $u_{1}<u_{3}<w_{3} \leqslant u_{2}$ in $T$, where $u_{i}:=u_{a_{i} b_{i}}$ and $w_{i}:=w_{a_{i} b_{i}}$ for each $i \in\{1,2,3\}$. Then

(i) $x_{1} \leqslant z_{3} \leqslant y_{2}$, and

(ii) $y_{1} \leqslant y_{3} \leqslant z_{2} \leqslant b_{1}$

in $P$, where $x_{i}:=x_{a_{i} b_{i}}, y_{i}:=y_{a_{i} b_{i}}$, and $z_{i}:=z_{a_{i} b_{i}}$ for each $i \in\{1,2,3\}$.

Proof Let $p_{i}:=p_{a_{i} b_{i}}$ for each $i \in\{1,2,3\}$. We have $x_{1} \leqslant y_{2}$ and $y_{1} \leqslant z_{2} \leqslant b_{1}$ in $P$ by Claim 18. Since $u_{1}<u_{3}<w_{3} \leqslant u_{2}$ in $T$, the relations $x_{1} \leqslant y_{2}$ and $y_{1} \leqslant z_{2}$ hit $B\left(u_{3}\right) \cap B\left(w_{3}\right)=\left\{x_{3}, y_{3}\right\}$. They cannot hit the same element since otherwise $a_{1} \leqslant x_{1} \leqslant$ $z_{2} \leqslant b_{1}$ in $P$.

If $x_{1} \leqslant y_{3} \leqslant y_{2}$ in $P$ then $y_{1} \leqslant x_{3} \leqslant z_{2}$, and we conclude $a_{1} \leqslant x_{1} \leqslant y_{3} \leqslant b_{3}$ and $a_{3} \leqslant x_{3} \leqslant z_{2} \leqslant b_{1}$ in $P$. Thus, $\left(a_{1}, b_{1}\right)$ and $\left(a_{3}, b_{3}\right)$ form an alternating cycle of length 2. Applying Claim 16 on the pairs $\left(a_{1}, b_{1}\right),\left(a_{2}, b_{2}\right)$ we obtain in particular $u_{2}<b_{1}^{T}$ in $T$. Together with our assumptions it follows that both $u_{3}$ and $w_{3}$ lie on the path from $u_{1}$ to $b_{1}^{T}$ in $T$. Hence, $u_{1}<w_{1} \leqslant u_{3}<w_{3}<b_{1}^{T}$ in $T$, and therefore $\left(a_{1}, b_{1}\right),\left(a_{3}, b_{3}\right)$ is a special 2-cycle, contradicting the fact that $\psi_{8, \Sigma}\left(a_{1}, b_{1}\right)=\alpha_{8}\left(a_{1}, b_{1}\right)=\alpha_{8}\left(a_{3}, b_{3}\right)=\psi_{8, \Sigma}\left(a_{3}, b_{3}\right)$.

We conclude that $x_{1} \leqslant x_{3} \leqslant y_{2}$ and $y_{1} \leqslant y_{3} \leqslant z_{2}$ in $P$. It remains to show that $x_{1} \leqslant z_{3} \leqslant y_{2}$ in $P$. For this, note that $x_{1} \leqslant x_{3}$ hits $B\left(p_{3}\right) \cap B\left(u_{3}\right)=\left\{y_{3}, z_{3}\right\}$. It cannot hit $y_{3}$ as otherwise $a_{1} \leqslant x_{1} \leqslant y_{3} \leqslant z_{2} \leqslant b_{1}$ in $P$. This implies $x_{1} \leqslant z_{3} \leqslant x_{3} \leqslant y_{2}$ in $P$, as desired.

We are now ready to prove our main claim about $K_{\Sigma}\left(\Sigma \in \Sigma\left(v_{9}\right)\right)$, namely that $K_{\Sigma}$ is bipartite. (We consider a directed graph to be bipartite if its underlying undirected graph is.) 
Claim 20 The graph $K_{\Sigma}$ is bipartite for each $\Sigma \in \Sigma\left(v_{9}\right)$.

Proof Arguing by contradiction, suppose that there is an odd undirected cycle $C=$ $\left\{\left(a_{i}, b_{i}\right)\right\}_{i=1}^{k}$ in $K_{\Sigma}$ for some $\Sigma \in \Sigma\left(v_{9}\right)$. Let $u_{i}:=u_{a_{i} b_{i}}, w_{i}:=w_{a_{i} b_{i}}, x_{i}:=x_{a_{i} b_{i}}$, $y_{i}:=y_{a_{i} b_{i}}$, and $z_{i}:=z_{a_{i} b_{i}}$ for each $i \in\{1, \ldots, k\}$. We may assume that $\alpha_{1}\left(a_{i}, b_{i}\right)=$ left for each $i \in\{1, \ldots, k\}$.

Consider the cyclic sequence of nodes $\left(u_{1}, u_{2}, \ldots, u_{k}\right)$. It might be the case that some of the nodes coincide. In order to avoid this, we modify the sequence as follows: If $u_{i}=u_{j}$ for some $i, j \in\{1, \ldots, k\}$ with $i<j$, then consider the two cyclic sequences $\left(u_{i}, u_{i+1}, \ldots, u_{j-1}\right)$ and $\left(u_{j}, u_{j+1}, \ldots, u_{i-1}\right)$ (thus the second one contains $u_{k}$, and also $u_{1}$ if $i>1$ ). Since $k$ is odd, exactly one of the two cyclic sequences has odd length. We replace the original sequence by that one, and repeat this process as long as some node appears at least twice in the current cyclic sequence.

We claim that at every stage of the above modification process the cyclic sequence $S=\left(u_{i_{1}}, u_{i_{2}}, \ldots, u_{i_{\ell}}\right)$ under consideration satisfies the following property: For every $s \in\{1, \ldots, \ell\}$ there is an index $j \in\{1, \ldots, k\}$ such that $u_{i_{s}}=u_{j}$ and $u_{i_{s+1}}=u_{j+1}$ (taking indices cyclically in each case, as expected). This property obviously holds at the start, so let us show that it remains true during the rest of the procedure. Thus suppose that the current cyclic sequence $S=\left(u_{i_{1}}, u_{i_{2}}, \ldots, u_{i_{\ell}}\right)$ satisfies the property, and that we modify it because of two indices $p, q \in\{1, \ldots, \ell\}$ with $p<q$ such that $u_{i_{p}}=u_{i_{q}}$. Without loss of generality we may assume that the resulting odd sequence is $S^{\prime}=\left(u_{i_{p}}, \ldots, u_{i_{q-1}}\right)$. We only need to show that there is an index $j \in\{1, \ldots, k\}$ such that $u_{i_{q-1}}=u_{j}$ and $u_{i_{p}}=u_{j+1}$, since $u_{i_{q-1}}$ and $u_{i_{p}}$ are the only two consecutive nodes in $S^{\prime}$ that were not consecutive in $S$. Then it suffices to take $j \in\{1, \ldots, k\}$ such that $u_{i_{q-1}}=u_{j}$ and $u_{i_{q}}=u_{j+1}$, which exists since $S$ satisfies our property, and observe that $u_{i_{p}}=u_{i_{q}}=u_{j+1}$. Therefore, the property holds at every step, as claimed.

Recalling that any two consecutive nodes in the original cyclic sequence $\left(u_{1}, u_{2}, \ldots, u_{k}\right)$ are distinct and comparable in $T$ (by Claim 16), it follows from the property considered above that this holds at every step of the modification procedure, and thus in particular for the final sequence $S=\left(u_{i_{1}}, u_{i_{2}}, \ldots, u_{i_{\ell}}\right)$ resulting from the procedure. In particular, $\ell \geqslant 3$, because the cycle has odd length.

Since $\ell$ is odd, there exists $m \in\{1, \ldots, \ell\}$ such that $u_{i_{m-1}}<u_{i_{m}}<u_{i_{m+1}}$ or $u_{i_{m-1}}>$ $u_{i_{m}}>u_{i_{m+1}}$ in $T$. Reversing the ordering of $C$ and the cyclic sequence $S$ if necessary, we may assume without loss of generality $u_{i_{m-1}}<u_{i_{m}}<u_{i_{m+1}}$ in $T$. Similarly, shifting the sequence $S$ cyclically if necessary, we may assume $m=1$. Thus $u_{i_{\ell}}<u_{i_{1}}<u_{i_{2}}$ in $T$.

Let $w$ be the neighbor of $u_{i_{1}}$ on the $u_{i_{1}}-u_{i_{2}}$ path in $T$. Thus $w \leqslant u_{i_{2}}$ in $T$. Now let $n \in\{3, \ldots, \ell\}$ be minimal such that $w \nless u_{i_{n}}$ in $T$. Since $u_{i_{\ell}}<w$ in $T$, this index exists. As $u_{i_{n-1}}$ and $u_{i_{n}}$ are comparable in $T$, it follows that $u_{i_{n}}<w \leqslant u_{i_{n-1}}$ in $T$. (This follows from the definition of $n$ if $n>3$, and from the fact that $w \leqslant u_{i_{2}}$ in $T$ if $n=3$.) Furthermore we have $u_{i_{n}} \neq u_{i_{1}}$, because $n \neq 1$ and all nodes in $S$ are distinct. We conclude that

$$
u_{i_{n}}<u_{i_{1}}<w \leqslant u_{i_{n-1}}
$$

in $T$. Now, by the property that is maintained during the modification process of $S$, there exist indices $r, s, t \in\{1, \ldots, k\}$ such that

$$
\begin{aligned}
& u_{i_{\ell}}=u_{r} \quad \text { and } \quad u_{i_{1}}=u_{r+1}, \\
& u_{i_{1}}=u_{s} \quad \text { and } \quad u_{i_{2}}=u_{s+1} \text {, } \\
& u_{i_{n-1}}=u_{t} \quad \text { and } \quad u_{i_{n}}=u_{t+1} \text {. }
\end{aligned}
$$


It follows that $K_{\Sigma}$ contains the following arcs: $\left(\left(a_{r}, b_{r}\right),\left(a_{r+1}, b_{r+1}\right)\right),\left(\left(a_{s}, b_{s}\right),\left(a_{s+1}\right.\right.$, $\left.\left.b_{s+1}\right)\right)$, and $\left(\left(a_{t+1}, b_{t+1}\right),\left(a_{t}, b_{t}\right)\right)$. Applying Claim 16 on these arcs we obtain:

$$
\begin{gathered}
u_{r}<w_{r} \leqslant u_{r+1}<b_{r}^{T}, \\
u_{s}<w_{s} \leqslant u_{s+1}<b_{s}^{T}, \\
u_{t+1}<w_{t+1} \leqslant u_{t}<b_{t+1}^{T}
\end{gathered}
$$

in $T$. See Fig. 8 for a possible configuration in $T$ and for upcoming arguments. Since $u_{i_{1}}=$ $u_{s}$ and $u_{i_{2}}=u_{s+1}$ we have $w=w_{s}$. Hence with (9) we get $u_{t+1}<u_{s}<w_{s} \leqslant u_{t}$ in $T$, and by Claim 19 it follows that

$$
x_{t+1} \leqslant z_{s} \leqslant y_{t} \text { and } y_{t+1} \leqslant y_{s} \leqslant z_{t} \leqslant b_{t+1}
$$

in $P$. Now applying Claim 18 on the $\operatorname{arc}\left(\left(a_{r}, b_{r}\right),\left(a_{r+1}, b_{r+1}\right)\right)$ we get

$$
x_{r} \leqslant y_{r+1} \text { and } y_{r} \leqslant z_{r+1} \leqslant b_{r}
$$

in $P$. Since $\alpha_{5}\left(a_{r+1}, b_{r+1}\right)=\alpha_{5}\left(a_{s}, b_{s}\right)$ and $B\left(u_{r+1}\right)=B\left(u_{s}\right)$ (because $u_{r+1}=u_{s}$ ), we conclude that $y_{r+1}=y_{s}$ and $z_{r+1}=z_{s}$. Using this and the derived inequalities we see that

$$
a_{t+1} \leqslant x_{t+1} \leqslant z_{s}=z_{r+1} \leqslant b_{r} \quad \text { and } \quad a_{r} \leqslant x_{r} \leqslant y_{r+1}=y_{s} \leqslant b_{t+1}
$$

in $P$. Thus, $\left(a_{r}, b_{r}\right)$ and $\left(a_{t+1}, b_{t+1}\right)$ form an alternating cycle of length 2 . In particular, this shows $r \neq t+1$ (otherwise we would have $a_{r} \leqslant b_{t+1}=b_{r}$ in $P$ ), and consequently (by Claim 16)

$$
u_{r} \neq u_{t+1} \text {. }
$$

Observe that $u_{r}<u_{r+1}=u_{i_{1}}$ (by (13) and (10)) and $u_{t+1}=u_{i_{n}}<u_{i_{1}}$ (by (12) and (9)) in $T$, which makes $u_{r}$ and $u_{t+1}$ comparable in $T$. Furthermore,

$$
u_{i_{1}} \stackrel{(10)}{=} u_{r+1} \stackrel{(13)}{<} b_{r}^{T} \quad \text { and } \quad u_{i_{1}} \stackrel{(9)}{<} u_{i_{n-1}} \stackrel{(12)}{=} u_{t} \stackrel{(15)}{<} b_{t+1}^{T}
$$

in $T$, so all together we have

$$
\left\{u_{r}, u_{t+1}\right\}<u_{i_{1}}<\left\{b_{r}^{T}, b_{t+1}^{T}\right\}
$$

in $T$. But from this and (16) it follows that either $u_{r}<w_{r} \leqslant u_{t+1}<w_{t+1} \leqslant u_{i_{1}}<b_{r}^{T}$ or $u_{t+1}<w_{t+1} \leqslant u_{r}<w_{r} \leqslant u_{i_{1}}<b_{t+1}^{T}$ holds in $T$. Both cases imply that $\left(a_{r}, b_{r}\right)$ and $\left(a_{t+1}, b_{t+1}\right)$ form a special 2-cycle, which is our final contradiction.

Using Claim 20 we let $\psi_{9, \Sigma}: \operatorname{MM}\left(P, v_{9}, \Sigma\right) \rightarrow\{1,2\}$ be a 2-coloring of $K_{\Sigma}$, for each $\Sigma \in \Sigma\left(\nu_{9}\right)$. The function $\alpha_{9}$ then records the color of a pair in this coloring:

For each $\Sigma \in \boldsymbol{\Sigma}\left(\nu_{9}\right)$ and each pair $(a, b) \in \operatorname{MM}\left(P, \nu_{9}, \Sigma\right)$, we let

$$
\alpha_{9}(a, b):=\psi_{9, \Sigma}(a, b) \text {. }
$$

\subsection{Third leaf of $\Psi: v_{10}$}

Now suppose that there was a strict alternating cycle $\left\{\left(a_{i}, b_{i}\right)\right\}_{i=1}^{k}$ with root $\left(a_{1}, b_{1}\right)$ in $\operatorname{MM}\left(P, v_{10}, \Sigma\right)$, for some $\Sigma \in \Sigma\left(v_{10}\right)$. Then, by the definition of $K_{\Sigma}$, there is an arc from $\left(a_{1}, b_{1}\right)$ to $\left(a_{2}, b_{2}\right)$ in $K_{\Sigma}$, and hence $\alpha_{9}\left(a_{1}, b_{1}\right) \neq \alpha_{9}$, a contradiction. Therefore, there is no such cycle in $\operatorname{MM}\left(P, v_{10}, \Sigma\right)$, and we have established the following claim:

Claim 21 The set $\operatorname{MM}\left(P, v_{10}, \Sigma\right)$ is reversible for each $\Sigma \in \Sigma\left(v_{10}\right)$. 


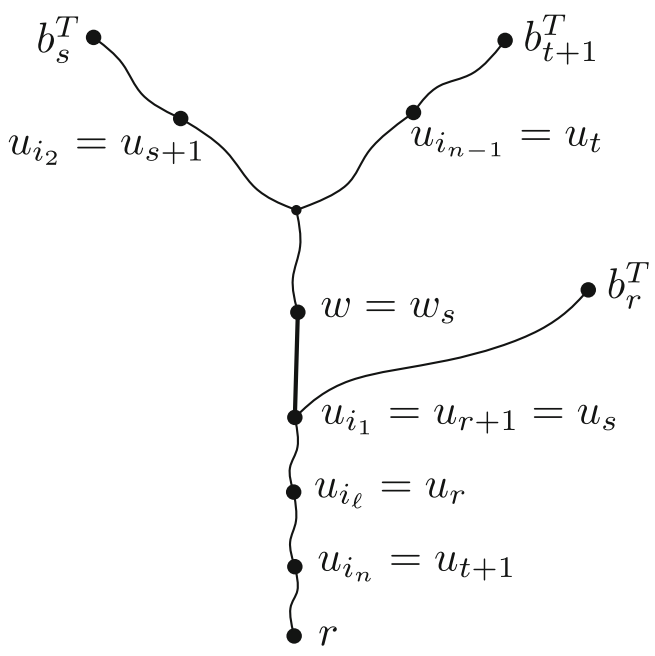

Fig. 8 Possible situation in proof of Claim 20. Note that we could also have $u_{r}<u_{t+1}$ in $T$

This concludes our study of the third leaf of $\Psi$.

\subsection{Node $v_{11}$ and its functions $\alpha_{11}$}

In this section we consider pairs $(a, b) \in \operatorname{MM}\left(P, v_{11}\right)$. Recall that in this case $(a, b)$ satisfies $\alpha_{2}(a, b)=$ yes and even stronger $\alpha_{4}(a, b)=$ yes, and hence there is $q \in$ $B\left(u_{a b}\right) \cap B\left(p_{a b}\right)$ such that $a \leqslant q$ in $P$.

For every such pair $(a, b)$, denote the three elements in $B\left(u_{a b}\right)$ as $x_{a b}, y_{a b}, z_{a b}$ in such a way that

$$
B\left(u_{a b}\right) \cap B\left(p_{a b}\right)=\left\{x_{a b}, y_{a b}\right\}
$$

and

$$
\begin{aligned}
& a \leqslant x_{a b} \nless b ; \\
& a \nless y_{a b} \leqslant b
\end{aligned}
$$

in $P$. (Here we use that $\alpha_{4}(a, b)=$ yes and that $a_{0} \leqslant b$ hits $B\left(u_{a b}\right) \cap B\left(p_{a b}\right)$.)

The function $\alpha_{11}$ is defined similarly as $\alpha_{5}$ in Section 3.5:

For each $\Sigma \in \mathbf{\Sigma}\left(\nu_{11}\right)$ and pair $(a, b) \in \operatorname{MM}\left(P, \nu_{11}, \Sigma\right)$, we let

$$
\alpha_{11}(a, b):=\left(\phi\left(x_{a b}\right), \phi\left(y_{a b}\right), \phi\left(z_{a b}\right)\right) .
$$

(Recall that $\phi$ is the 3-coloring of $X$ defined earlier on which is such that $x, y \in X$ receive distinct colors whenever $V\left(T_{x}\right) \cap V\left(T_{y}\right) \neq \varnothing$; in particular, the three colors $\phi\left(x_{a b}\right), \phi\left(y_{a b}\right), \phi\left(z_{a b}\right)$ are distinct. $)$

\subsection{Node $v_{12}$ and its functions $\alpha_{12}$}

We start by defining function $\alpha_{12}$ : 
For each $\Sigma \in \boldsymbol{\Sigma}\left(\nu_{12}\right)$ and pair $(a, b) \in \mathrm{MM}\left(P, \nu_{12}, \Sigma\right)$, we let $\alpha_{12}(a, b)$ record the three yes/no answers to three independent questions about the pair $(a, b)$, namely

$$
\begin{aligned}
& \text { "Is } a \leqslant z_{a b} \text { in } P \text { ?"; } \\
& \text { "Is } z_{a b} \leqslant b \text { in } P \text { ?"; } \\
& \text { "Is } a_{0} \leqslant x_{a b} \text { in } P \text { ?". }
\end{aligned}
$$

(Recall that $a_{0}<b$ in $P$ for all $b \in \max (P)$ ). Formally speaking, $\alpha_{12}(a, b)$ is defined as the vector $\left(s_{1}, s_{2}, s_{3}\right) \in\{\text { yes, no }\}^{3}$ where $s_{i}$ is the answer to the $i$-th question above, for $i=1,2,3$. Note that we cannot have $a \leqslant z_{a b}$ and $z_{a b} \leqslant b$ at the same time in $P$, and thus there are only 6 possible vectors of answers. (This is why the corresponding edge in the signature tree $\Psi$ is labeled 6 instead of 8.)

\subsection{Node $\nu_{13}$ and its functions $\alpha_{13}$ : Dealing with strict alternating cycles of length 2}

Let us first summarize the properties of pairs in $\operatorname{MM}\left(P, v_{13}\right)$. For each $\Sigma \in \Sigma\left(v_{13}\right)$ and each $(a, b) \in \operatorname{MM}\left(P, v_{13}, \Sigma\right)$ we have that

- $\quad$ elements of $B\left(u_{a b}\right)$ can be labeled with $x_{a b}, y_{a b}, z_{a b}$ such that (17)-(19) hold,

- $\phi\left(x_{a b}\right), \phi\left(y_{a b}\right), \phi\left(z_{a b}\right)$ are pairwise distinct,

- $\phi\left(x_{a b}\right)=\phi\left(x_{a^{\prime} b^{\prime}}\right), \phi\left(y_{a b}\right)=\phi\left(y_{a^{\prime} b^{\prime}}\right)$ and $\phi\left(z_{a b}\right)=\phi\left(z_{a^{\prime} b^{\prime}}\right)$ for every $\left(a^{\prime}, b^{\prime}\right) \in$ $\operatorname{MM}\left(P, v_{13}, \Sigma\right)$,

- $\quad$ all pairs of $\operatorname{MM}\left(P, v_{13}, \Sigma\right)$ produce the same answers to Q1-Q2.

For each $\Sigma \in \Sigma\left(v_{13}\right)$ let $J_{\Sigma}$ be the graph with vertex set $\operatorname{MM}\left(P, v_{13}, \Sigma\right)$ where two distinct pairs $(a, b),\left(a^{\prime}, b^{\prime}\right) \in \operatorname{MM}\left(P, v_{13}, \Sigma\right)$ are adjacent if and only if $(a, b),\left(a^{\prime}, b^{\prime}\right)$ is an alternating cycle. Our goal in this section is to show that $J_{\Sigma}$ is 4-colorable:

Lemma 22 For each $\Sigma \in \Sigma\left(v_{13}\right)$ there is a proper coloring of $J_{\Sigma}$ with 4 colors.

To this aim, we show a number of properties of 2-cycles in $\operatorname{MM}\left(P, v_{13}, \Sigma\right)$.

Claim 23 Let $\Sigma \in \Sigma\left(v_{13}\right)$ and suppose that $\left(a_{1}, b_{1}\right),\left(a_{2}, b_{2}\right)$ is a 2-cycle in $\operatorname{MM}\left(P, v_{13}, \Sigma\right)$. Let $u_{i}:=u_{a_{i} b_{i}}$ for $i=1,2$. Then $u_{1} \neq u_{2}$.

Proof Let $x_{i}:=x_{a_{i} b_{i}}, y_{i}:=y_{a_{i} b_{i}}$, and $z_{i}:=z_{a_{i} b_{i}}$ for $i=1,2$. We may assume $\alpha_{1}\left(a_{i}, b_{i}\right)=$ left for $i=1,2$. Arguing by contradiction suppose that $u_{1}=u_{2}$. Exchanging $\left(a_{1}, b_{1}\right)$ and $\left(a_{2}, b_{2}\right)$ if necessary we may assume that $b_{1}^{T}$ is left of $b_{2}^{T}$ in $T$.

Since in $T$ the node $a_{1}^{T}$ is left of $b_{1}^{T}$, which itself is left of $b_{2}^{T}$, the path connecting $a_{1}^{T}$ to $b_{2}^{T}$ in $T$ goes through $u_{1}$. Thus, the relation $a_{1} \leqslant b_{2}$ hits $B\left(u_{1}\right)=\left\{x_{1}, y_{1}, z_{1}\right\}=B\left(u_{2}\right)=$ $\left\{x_{2}, y_{2}, z_{2}\right\}$, and hence $a_{1} \leqslant c \leqslant b_{2}$ in $P$ for some element $c \in B\left(u_{1}\right)$. Recall that $a_{i} \leqslant x_{i}$ and $a_{i} y_{i}$ in $P$ for $i=1,2$. Thus $c \in\left\{x_{1}, z_{1}\right\}$. Moreover, $\left(\phi\left(x_{1}\right), \phi\left(y_{1}\right), \phi\left(z_{1}\right)\right)=$ $\left(\phi\left(x_{2}\right), \phi\left(y_{2}\right), \phi\left(z_{2}\right)\right)$ since $\alpha_{11}\left(a_{1}, b_{1}\right)=\alpha_{11}\left(a_{2}, b_{2}\right)$, which implies $x_{1}=x_{2}, y_{1}=y_{2}$, and $z_{1}=z_{2}$.

If $c=x_{1}$ then $a_{2} \leqslant x_{2}=x_{1} \leqslant b_{2}$ in $P$, a contradiction. If $c=z_{1}$ then, using that $a_{2} \leqslant z_{2}$ in $P\left(\right.$ since $\left.\alpha_{12}\left(a_{1}, b_{1}\right)=\alpha_{12}\left(a_{2}, b_{2}\right)\right)$, we obtain $a_{2} \leqslant z_{2}=z_{1} \leqslant b_{2}$ in $P$, again a contradiction. 
By Claim 23 if $\left(a_{1}, b_{1}\right),\left(a_{2}, b_{2}\right)$ is a 2 -cycle in $\operatorname{MM}\left(P, v_{13}, \Sigma\right)$ for some $\Sigma \in \Sigma\left(v_{13}\right)$ then $u_{a_{1} b_{1}} \neq u_{a_{2} b_{2}}$. Let us say that the 2-cycle is of type 1 if the latter two nodes are comparable in $T$ (that is, $u_{a_{1} b_{1}}<u_{a_{2} b_{2}}$ or $u_{a_{1} b_{1}}>u_{a_{2} b_{2}}$ in $T$ ), and of type 2 otherwise. By extension, each edge of the graph $J_{\Sigma}$ is either of type 1 or of type 2 . Let $J_{\Sigma, i}$ denote the spanning subgraph of $J_{\Sigma}$ defined by the edges of type $i$, for $i=1,2$. Thus $J_{\Sigma, 1}$ and $J_{\Sigma, 2}$ are edge disjoint, and $J_{\Sigma}=J_{\Sigma, 1} \cup J_{\Sigma, 2}$. In what follows we will first show that $J_{\Sigma, 1}$ is bipartite, and then considering a 2-coloring of $J_{\Sigma, 1}$, we will prove that the two subgraphs of $J_{\Sigma, 2}$ induced by the two color classes are bipartite. This clearly implies our main lemma, Lemma 22 , that $J_{\Sigma}$ is 4-colorable.

Claim 24 Let $\Sigma \in \Sigma\left(v_{13}\right)$ and suppose that $\left(a_{1}, b_{1}\right),\left(a_{2}, b_{2}\right)$ is a 2-cycle in $\operatorname{MM}\left(P, v_{13}, \Sigma\right)$ of type 1 . Let $u_{i}:=u_{a_{i} b_{i}}$ and $w_{i}:=w_{a_{i} b_{i}}$ for $i=1,2$, and suppose further that $u_{1}<u_{2}$ in $T$. Then $u_{1}<w_{1} \leqslant u_{2}$ in $T$.

Proof Arguing by contradiction, suppose that $w_{1} \nless u_{2}$ in $T$. Let $v_{i}:=v_{a_{i} b_{i}}, p_{i}:=\mathrm{p}\left(u_{i}\right)$, $x_{i}:=x_{a_{i} b_{i}}, y_{i}:=y_{a_{i} b_{i}}$, and $z_{i}:=z_{a_{i} b_{i}}$ for $i=1,2$.

First suppose that $v_{1} \leqslant u_{2}$ in $T$. Then the path from $a_{2}^{T}$ to $b_{1}^{T}$ in $T$ goes through $u_{2}, p_{2}, v_{1}$ and $u_{1}$. Thus the relation $a_{2} \leqslant b_{1}$ hits $B\left(u_{2}\right) \cap B\left(p_{2}\right)=\left\{x_{2}, y_{2}\right\}$ and $B\left(v_{1}\right) \cap B\left(u_{1}\right)$. Note that the two edges $u_{2} p_{2}$ and $v_{1} u_{1}$ may coincide (if $u_{2}=v_{1}$ ). Since $a_{2} \leqslant b_{1}$ cannot hit $y_{2}$ because $y_{2} \leqslant b_{2}$ in $P$, it hits $x_{2}$, and by Observation 10 we then have

$$
a_{2} \leqslant x_{2} \leqslant c \leqslant b_{1}
$$

in $P$ for some $c \in B\left(v_{1}\right) \cap B\left(u_{1}\right)$. Let $d$ be the element in $\left(B\left(v_{1}\right) \cap B\left(u_{1}\right)\right) \backslash\{c\}$.

The $a_{1}^{T}-u_{1}$ path and the $r-b_{2}^{T}$ path in $T$ both go through the edge $v_{1} u_{1}$. Thus, the relations $a_{1} \leqslant x_{1}$ and $a_{0} \leqslant b_{2}$ both hit $\{c, d\}$. Neither can hit $c$ since otherwise $a_{1} \leqslant c \leqslant b_{1}$ or $a_{2} \leqslant c \leqslant b_{2}$ in $P$. Hence, $a_{1} \leqslant d \leqslant x_{1}$ and $a_{0} \leqslant d \leqslant b_{2}$ in $P$, which implies $a_{0} \leqslant x_{1}$. We then have $a_{0} \leqslant x_{2}$ in $P$ as well, since by $\alpha_{12}\left(a_{1}, b_{1}\right)=\alpha_{12}\left(a_{2}, b_{2}\right)$ both pairs $\left(a_{1}, b_{1}\right),\left(a_{2}, b_{2}\right)$ give the same answer to question Q3.

Clearly, $a_{0} \leqslant x_{2}$ hits $\{c, d\}$. This relation cannot hit $d$ since otherwise $a_{1} \leqslant d \leqslant x_{2}$ and $x_{2} \leqslant b_{1}$ (by (20)) would imply $a_{1} \leqslant b_{1}$ in $P$. Thus $a_{0} \leqslant c \leqslant x_{2}$ in $P$. Given that $x_{2} \leqslant c$ in $P$ by (20), we conclude $x_{2}=c$. Using that $x_{1}=c \in B\left(u_{1}\right)=\left\{x_{1}, y_{1}, z_{1}\right\}$ and $\left(\phi\left(x_{1}\right), \phi\left(y_{1}\right), \phi\left(z_{1}\right)\right)=\left(\phi\left(x_{2}\right), \phi\left(y_{2}\right), \phi\left(z_{2}\right)\right)$ (since $\left.\alpha_{11}\left(a_{1}, b_{1}\right)=\alpha_{11}\left(a_{2}, b_{2}\right)\right)$, we further deduce that $x_{1} \neq y_{2}$ (because $\left.\phi\left(x_{1}\right)=\phi\left(x_{2}\right) \neq \phi\left(y_{2}\right)\right)$ and $x_{1} \neq z_{2}$ (because $\left.\phi\left(x_{1}\right)=\phi\left(x_{2}\right) \neq \phi\left(z_{2}\right)\right)$ and therefore $x_{2}=c=x_{1}$. However, this implies $a_{1} \leqslant x_{1}=$ $x_{2} \leqslant b_{1}$ in $P$, a contradiction.

Next assume that $v_{1} \nless u_{2}$ in $T$. Let $v_{1}^{\prime}$ be the neighbor of $u_{1}$ on the $u_{1}-u_{2}$ path in $T$. Thus $v_{1}^{\prime} \neq w_{1}$ and $v_{1}^{\prime} \neq v_{1}$. The path from $a_{2}^{T}$ to $b_{1}^{T}$ in $T$ goes through $u_{2}, p_{2}, v_{1}^{\prime}$ and $u_{1}$. Thus, the relation $a_{2} \leqslant b_{1}$ hits $B\left(p_{2}\right) \cap B\left(u_{2}\right)=\left\{x_{2}, y_{2}\right\}$ and $B\left(v_{1}^{\prime}\right) \cap B\left(u_{1}\right)$. It cannot hit $y_{2}$ since otherwise $a_{2} \leqslant y_{2} \leqslant b_{2}$ in $P$. By Observation 10, we then have

$$
a_{2} \leqslant x_{2} \leqslant c^{\prime} \leqslant b_{1}
$$

for some $c^{\prime} \in B\left(v_{1}^{\prime}\right) \cap B\left(u_{1}\right)$. Let $d^{\prime}$ be the element in $\left(B\left(v_{1}^{\prime}\right) \cap B\left(u_{1}\right)\right) \backslash\left\{c^{\prime}\right\}$.

The paths from $r$ to $b_{2}^{T}$ and from $a_{1}^{T}$ to $b_{2}^{T}$ in $T$ both go through $u_{1}$ and $v_{1}^{\prime}$. Thus the two relations $a_{0} \leqslant b_{2}$ and $a_{1} \leqslant b_{2}$ hit the set $\left\{c^{\prime}, d^{\prime}\right\}$. They cannot hit $c^{\prime}$ since otherwise we would get $c^{\prime} \leqslant b_{2}$, implying $a_{2} \leqslant c^{\prime} \leqslant b_{2}$ in $P$ by (21). Hence, $a_{0} \leqslant d^{\prime} \leqslant b_{2}$ and $a_{1} \leqslant d^{\prime} \leqslant b_{2}$ in $P$.

Observe that $\left\{c^{\prime}, d^{\prime}\right\} \subseteq\left\{x_{1}, y_{1}, z_{1}\right\}=B\left(u_{1}\right)$, and that we have $c^{\prime} \neq x_{1}$ (otherwise $a_{1} \leqslant x_{1}=c^{\prime} \leqslant b_{1}$ in $P$ with (21)) and $d^{\prime} \neq y_{1}$ (otherwise $a_{1} \leqslant d^{\prime}=y_{1} \leqslant b_{1}$ in $P$ ). 
We claim that $a_{0} \leqslant x_{1}$ in $P$. If $d^{\prime}=x_{1}$ this is obvious, so suppose that $d^{\prime}=z_{1}$, which implies $c^{\prime}=y_{1}$. The relation $a_{0} \leqslant d^{\prime}$ clearly hits $B\left(p_{1}\right) \cap B\left(u_{1}\right)=\left\{x_{1}, y_{1}\right\}$. If it hits $x_{1}$, then $a_{0} \leqslant x_{1}$ in $P$. If, on the other hand, it hits $y_{1}$, then together with (21) we obtain $a_{2} \leqslant c^{\prime}=y_{1} \leqslant d^{\prime} \leqslant b_{2}$ in $P$, a contradiction. Hence $a_{0} \leqslant x_{1}$ in $P$, as claimed. We then have $a_{0} \leqslant x_{2}$ in $P$ as well, since by $\alpha_{12}\left(a_{1}, b_{1}\right)=\alpha_{12}\left(a_{2}, b_{2}\right)$ both pairs $\left(a_{1}, b_{1}\right),\left(a_{2}, b_{2}\right)$ give the same answer to question Q3.

The relation $a_{0} \leqslant x_{2}$ also hits $\left\{c^{\prime}, d^{\prime}\right\}$. It cannot hit $d^{\prime}$, since otherwise $a_{1} \leqslant d^{\prime} \leqslant x_{2} \leqslant b_{1}$ in $P$ by (21). Thus we have $a_{0} \leqslant c^{\prime} \leqslant x_{2}$ in $P$. Note that this yields $c^{\prime}=x_{2}$, since we had $x_{2} \leqslant c^{\prime}$ in $P$. Using that $c^{\prime} \in B\left(u_{1}\right)$ and $\left(\phi\left(x_{1}\right), \phi\left(y_{1}\right), \phi\left(z_{1}\right)\right)=\left(\phi\left(x_{2}\right), \phi\left(y_{2}\right), \phi\left(z_{2}\right)\right)$ (since $\alpha_{11}\left(a_{1}, b_{1}\right)=\alpha_{11}\left(a_{2}, b_{2}\right)$ ), we deduce that $x_{2}=c^{\prime}=x_{1}$. However, this implies $a_{1} \leqslant x_{1}=x_{2} \leqslant b_{1}$ in $P$, a contradiction.

Claim 25 Let $\Sigma \in \Sigma\left(v_{13}\right)$ and suppose that $\left(a_{1}, b_{1}\right),\left(a_{2}, b_{2}\right)$ is a 2-cycle in $\operatorname{MM}\left(P, v_{13}, \Sigma\right)$ of type 1. Let $u_{i}:=u_{a_{i} b_{i}}, x_{i}:=x_{a_{i} b_{i}}$, and $y_{i}:=y_{a_{i} b_{i}}$ for $i=1,2$, and suppose further that $u_{1}<u_{2}$ in T. Then

(i) $a_{1} \leqslant y_{2}$, and

(ii) $x_{2} \leqslant b_{1}$

in $P$.

Proof Let $p_{i}:=\mathrm{p}\left(u_{i}\right), v_{i}:=v_{a_{i} b_{i}}$ and $w_{i}:=w_{a_{i} b_{i}}$ for $i=1,2$. The path from $a_{1}^{T}$ to $b_{2}^{T}$ in $T$ has to go through $u_{1}$ since $u_{1}=\mathrm{p}\left(w_{1}\right), w_{1} \nless a_{1}^{T}$ and $w_{1} \leqslant u_{2}<b_{2}^{T}$ in $T$ (by Claim 24). As a consequence, this path goes through $p_{2}$ and $u_{2}$. Hence the relation $a_{1} \leqslant b_{2}$ hits $B\left(p_{2}\right) \cap B\left(u_{2}\right)=\left\{x_{2}, y_{2}\right\}$. It cannot hit $x_{2}$, since otherwise $a_{2} \leqslant x_{2} \leqslant b_{2}$ in $P$. Therefore, it hits $y_{2}$, showing (i).

To show (ii), observe that in $T$ at least one of the $r-b_{1}^{T}$ path and the $a_{2}^{T}-b_{1}^{T}$ path goes through $p_{2}$ and $u_{2}$. Thus, at least one of the two relations $a_{0} \leqslant b_{1}$ and $a_{2} \leqslant b_{1}$ hits $\left\{x_{2}, y_{2}\right\}$. Neither can hit $y_{2}$ since otherwise $a_{1} \leqslant y_{2} \leqslant b_{1}$ in $P$. Therefore, $x_{2} \leqslant b_{1}$ in $P$.

Claim 26 The graph $J_{\Sigma, 1}$ is triangle-free for each $\Sigma \in \Sigma\left(v_{13}\right)$.

Proof Let $\Sigma \in \Sigma\left(v_{13}\right)$. Arguing by contradiction, suppose that there is a triangle $\left(a_{1}, b_{1}\right),\left(a_{2}, b_{2}\right),\left(a_{3}, b_{3}\right)$ in $J_{\Sigma, 1}$.

Let $u_{i}:=u_{a_{i} b_{i}}, p_{i}:=\mathrm{p}\left(u_{i}\right), w_{i}:=w_{a_{i} b_{i}}, x_{i}:=x_{a_{i} b_{i}}$, and $y_{i}:=y_{a_{i} b_{i}}$ for each $i \in\{1,2,3\}$. Since the nodes $u_{1}, u_{2}, u_{3}$ are pairwise comparable in $T$ and are all distinct (by Claim 23), we may assume without loss of generality $u_{1}<u_{2}<u_{3}$ in $T$.

First we show that $a_{0} \leqslant x_{i}$ holds in $P$ for some $i \in\{1,2,3\}$. Suppose this is not the case. Consider the path from $r$ to $b_{3}^{T}$ in $T$. This path goes through the nodes $p_{1}, u_{1}, p_{2}, u_{2}, p_{3}$ and $u_{3}$. Hence, the relation $a_{0} \leqslant b_{3}$ hits $\left\{x_{1}, y_{1}\right\},\left\{x_{2}, y_{2}\right\}$ and $\left\{x_{3}, y_{3}\right\}$. By our assumption it hits $y_{i}$ for each $i \in\{1,2,3\}$, and we have $a_{0} \leqslant y_{1} \leqslant y_{2} \leqslant y_{3}$ in $P$ by Observation 10 .

If $u_{2} \leqslant b_{1}^{T}$ in $T$ then $a_{0} \leqslant b_{1}$ hits $\left\{x_{2}, y_{2}\right\}$, and thus hits $y_{2}$ by our assumption. Hence $y_{2} \leqslant b_{1}$ in $P$, which using Claim 25(i) implies $a_{1} \leqslant y_{2} \leqslant b_{1}$ in $P$, a contradiction. Therefore, $u_{2} \| b_{1}^{T}$ in $T$.

The fact that $u_{1}<u_{2}<u_{3}$ in $T$ further implies

$$
u_{1}<w_{1} \leqslant u_{2}<w_{2} \leqslant u_{3}
$$

by Claim 24. Observe that the $a_{1}^{T}-b_{2}^{T}$ path, the $a_{2}^{T}-b_{3}^{T}$ path, and the $a_{3}^{T}-b_{1}^{T}$ path in $T$ all include the edge $u_{2} w_{2}$. This is clear for the first two paths, and follows from $u_{2} \| b_{1}^{T}$ in $T$ for 
the third one. Thus the three relations $a_{1} \leqslant b_{2}, a_{2} \leqslant b_{3}, a_{3} \leqslant b_{1}$ all hit $B\left(u_{2}\right) \cap B\left(w_{2}\right)=$ : $\{c, d\}$. Without loss of generality we have $a_{1} \leqslant c \leqslant b_{2}$ in $P$. This implies $a_{2} \leqslant d \leqslant b_{3}$ in $P$, which in turn implies $a_{3} \leqslant c \leqslant b_{1}$. However, it follows that $a_{1} \leqslant c \leqslant b_{1}$ in $P$, a contradiction.

This shows that $a_{0} \leqslant x_{i}$ holds in $P$ for some $i \in\{1,2,3\}$, as claimed. Now, since $\alpha_{12}\left(a_{1}, b_{1}\right)=\alpha_{12}\left(a_{2}, b_{2}\right)=\alpha_{12}\left(a_{3}, b_{3}\right)$, it follows that $a_{0} \leqslant x_{i}$ in $P$ for each $i \in\{1,2,3\}$.

Consider the relation $a_{0} \leqslant x_{3}$ in $P$. The path from $r$ to $u_{3}$ in $T$ goes through $p_{2}$ and $u_{2}$. Thus, $a_{0} \leqslant x_{3}$ hits $\left\{x_{2}, y_{2}\right\}$. It cannot hit $x_{2}$ because otherwise $a_{2} \leqslant x_{2} \leqslant x_{3}$ in $P$, which together with $x_{3} \leqslant b_{2}$ (by Claim 25(i)) implies $a_{2} \leqslant x_{3} \leqslant b_{2}$ in $P$. Hence $a_{0} \leqslant x_{3}$ hits $y_{2}$, and we have $y_{2} \leqslant x_{3}$ in $P$. On the other hand, by Claim 25 we have $a_{1} \leqslant y_{2}$ and $x_{3} \leqslant b_{1}$ in $P$. It follows that $a_{1} \leqslant y_{2} \leqslant x_{3} \leqslant b_{1}$ in $P$, a contradiction. This concludes the proof.

Claim 27 Let $\Sigma \in \Sigma\left(v_{13}\right)$. Suppose that $\left(a_{1}, b_{1}\right),\left(a_{2}, b_{2}\right),\left(a_{3}, b_{3}\right) \in \operatorname{MM}\left(P, v_{13}, \Sigma\right)$ are three distinct pairs such that $\left(a_{1}, b_{1}\right),\left(a_{2}, b_{2}\right)$ form a 2 -cycle of type 1 and $\left(a_{1}, b_{1}\right),\left(a_{3}, b_{3}\right)$ do not form a 2-cycle (so neither of type 1 nor of type 2).

Let $u_{i}:=u_{a_{i} b_{i}}, x_{i}:=x_{a_{i} b_{i}}$, and $y_{i}:=y_{a_{i} b_{i}}$ for each $i \in\{1,2,3\}$. Assume further that $u_{1}<u_{3} \leqslant u_{2}$ in $T$. Then

(i) $a_{1} \leqslant x_{3}$, and

(ii) $y_{1} \leqslant y_{3} \leqslant b_{1}$

in $P$.

Proof Let $p_{i}:=\mathrm{p}\left(u_{i}\right)$ and $w_{i}:=w_{a_{i} b_{i}}$ for each $i \in\{1,2,3\}$. Since $u_{1}<u_{3} \leqslant u_{2}$ in $T$ and also $u_{1}<w_{1} \leqslant u_{2}$ in $T$ by Claim 24, it follows that

$$
u_{1}<w_{1} \leqslant u_{3} \leqslant u_{2}
$$

in $T$.

First suppose that $u_{3} \leqslant b_{1}^{T}$ in $T$. Then the relation $y_{1} \leqslant b_{1}$ hits $B\left(p_{3}\right) \cap B\left(u_{3}\right)=\left\{x_{3}, y_{3}\right\}$. By Claim 25(i) we have $a_{1} \leqslant y_{2}$ in $P$. Furthermore, $a_{1} \leqslant y_{2}$ also hits $\left\{x_{3}, y_{3}\right\}$. Clearly, $y_{1} \leqslant$ $b_{1}$ and $a_{1} \leqslant y_{2}$ cannot hit the same element of $\left\{x_{3}, y_{3}\right\}$. If $y_{1} \leqslant x_{3} \leqslant b_{1}$ then $a_{1} \leqslant y_{3} \leqslant y_{2}$ in $P$, which implies $a_{3} \leqslant x_{3} \leqslant b_{1}$ and $a_{1} \leqslant y_{3} \leqslant b_{3}$, that is, that $\left(a_{1}, b_{1}\right),\left(a_{3}, b_{3}\right)$ is a 2-cycle, a contradiction. Hence we have $y_{1} \leqslant y_{3} \leqslant b_{1}$ and $a_{1} \leqslant x_{3} \leqslant y_{2}$ in $P$, as claimed.

Next assume that $u_{3} b_{1}^{T}$ in $T$. Then the path from $a_{2}^{T}$ to $b_{1}^{T}$ in $T$ includes the edge $u_{3} p_{3}$, and hence $a_{2} \leqslant b_{1}$ hits $B\left(p_{3}\right) \cap B\left(u_{3}\right)=\left\{x_{3}, y_{3}\right\}$. The relation $a_{1} \leqslant$ $b_{2}$ also hits $\left\{x_{3}, y_{3}\right\}$. Clearly, $a_{2} \leqslant b_{1}$ and $a_{1} \leqslant b_{2}$ cannot hit the same element of $\left\{x_{3}, y_{3}\right\}$.

If $a_{2} \leqslant x_{3} \leqslant b_{1}$ in $P$ then $a_{1} \leqslant y_{3} \leqslant b_{2}$. However, it then follows $a_{3} \leqslant x_{3} \leqslant b_{1}$ and $a_{1} \leqslant y_{3} \leqslant b_{3}$ in $P$, implying that $\left(a_{1}, b_{1}\right),\left(a_{3}, b_{3}\right)$ is a 2 -cycle, a contradiction.

Therefore, $a_{2} \leqslant y_{3} \leqslant b_{1}$ and $a_{1} \leqslant x_{3} \leqslant b_{2}$ in $P$. In order to conclude the proof, it only remains to show that $y_{1} \leqslant y_{3}$ in $P$. For this, observe that the path from $r$ to $u_{3}$ in $T$ includes the edge $p_{1} u_{1}$. Hence, the relation $a_{0} \leqslant y_{3}$ hits $\left\{x_{1}, y_{1}\right\}$. It cannot hit $x_{1}$ since otherwise $a_{1} \leqslant x_{1} \leqslant y_{3} \leqslant b_{1}$. Thus, $a_{0} \leqslant y_{1} \leqslant y_{3}$ in $P$, as desired.

An illustration for the next two claims is given on Fig. 9.

Claim 28 Let $\Sigma \in \Sigma\left(v_{13}\right)$. Suppose that $\left(a_{1}, b_{1}\right),\left(a_{2}, b_{2}\right),\left(a_{3}, b_{3}\right),\left(a_{4}, b_{4}\right) \in$ $\operatorname{MM}\left(P, v_{13}, \Sigma\right)$ are four distinct pairs such that $u_{1}<u_{4}<u_{2}$ and $u_{3}<u_{4}$ in $T$, where $u_{i}:=u_{a_{i} b_{i}}$ for each $i \in\{1,2,3,4\}$. Assume further that $\left(a_{1}, b_{1}\right),\left(a_{2}, b_{2}\right)$ 


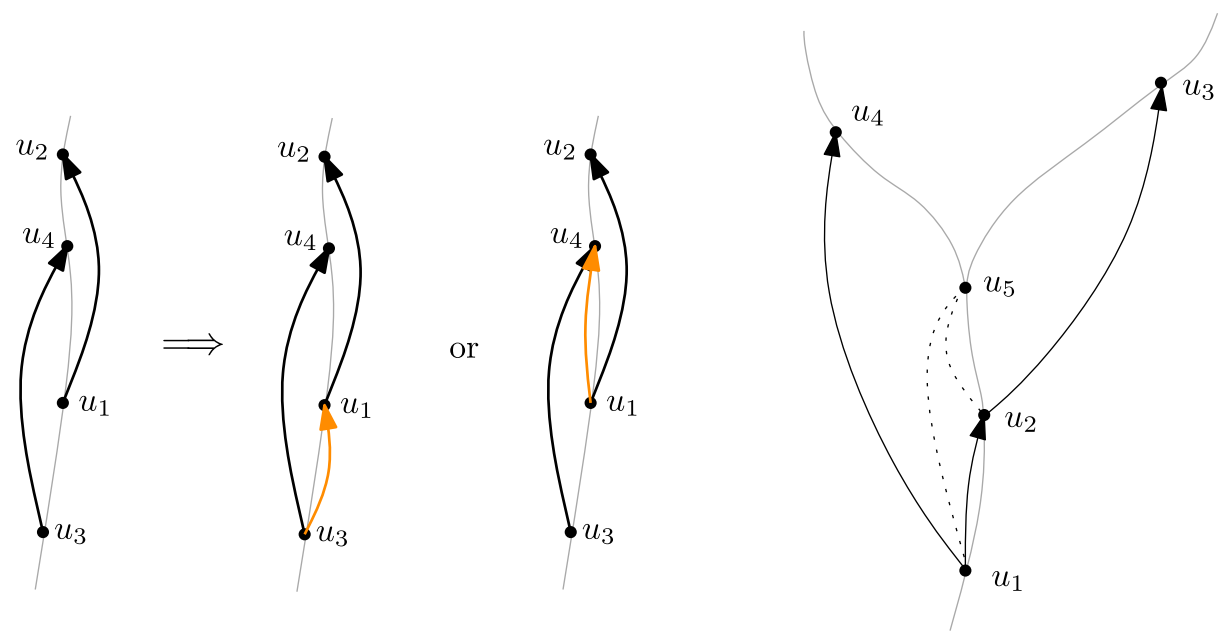

Fig. 9 Possible situations in Claim 28 and 29. An edge indicates that its endpoints correspond to the meeting points of a 2-cycle

and $\left(a_{3}, b_{3}\right),\left(a_{4}, b_{4}\right)$ are 2-cycles (which are thus of type 1). Then at least one of $\left(a_{1}, b_{1}\right),\left(a_{3}, b_{3}\right)$ and $\left(a_{1}, b_{1}\right),\left(a_{4}, b_{4}\right)$ is a 2-cycle of type 1 .

Proof Let $x_{i}:=x_{a_{i} b_{i}}$ and $y_{i}:=y_{a_{i} b_{i}}$ for each $i \in\{1,2,3,4\}$. Suppose that $\left(a_{1}, b_{1}\right),\left(a_{4}, b_{4}\right)$ is not a 2-cycle, since otherwise we are done. Applying Claim 27 on the three pairs $\left(a_{1}, b_{1}\right),\left(a_{2}, b_{2}\right),\left(a_{4}, b_{4}\right)$ we obtain that $a_{1} \leqslant x_{4}$ and $y_{1} \leqslant y_{4} \leqslant b_{1}$ in $P$.

Using Claim 25 on the 2-cycle $\left(a_{3}, b_{3}\right),\left(a_{4}, b_{4}\right)$ we also obtain $a_{3} \leqslant y_{4}$ and $x_{4} \leqslant b_{3}$ in $P$. It follows that $a_{3} \leqslant y_{4} \leqslant b_{1}$ and $a_{1} \leqslant x_{4} \leqslant b_{3}$ in $P$. Hence $\left(a_{1}, b_{1}\right),\left(a_{3}, b_{3}\right)$ is a 2 cycle. Furthermore, it is of type 1 because $u_{1}<u_{4}$ and $u_{3}<u_{4}$ in $T$, implying that $u_{1}$ and $u_{3}$ are comparable in $T$.

Claim 29 Let $\Sigma \in \Sigma\left(v_{13}\right)$. Suppose that $\left(a_{1}, b_{1}\right),\left(a_{2}, b_{2}\right),\left(a_{3}, b_{3}\right),\left(a_{4}, b_{4}\right),\left(a_{5}, b_{5}\right) \in$ $\operatorname{MM}\left(P, v_{13}, \Sigma\right)$ are five distinct pairs such that $u_{1}<u_{5}<u_{4}$ and $u_{2}<u_{5}<u_{3}$ in $T$, where $u_{i}:=u_{a_{i} b_{i}}$ for each $i \in\{1, \ldots, 5\}$. Assume further that $\left(a_{1}, b_{1}\right),\left(a_{2}, b_{2}\right)$ and $\left(a_{2}, b_{2}\right),\left(a_{3}, b_{3}\right)$ and $\left(a_{1}, b_{1}\right),\left(a_{4}, b_{4}\right)$ are 2-cycles of type 1. Then at least one of $\left(a_{1}, b_{1}\right),\left(a_{5}, b_{5}\right)$ and $\left(a_{2}, b_{2}\right),\left(a_{5}, b_{5}\right)$ is a 2-cycle of type 1 .

Proof Assume to the contrary that neither $\left(a_{1}, b_{1}\right),\left(a_{5}, b_{5}\right)$ nor $\left(a_{2}, b_{2}\right),\left(a_{5}, b_{5}\right)$ is a 2 cycle. (Note that if one is a 2-cycle, then it is automatically of type 1 since $u_{1}<u_{5}$ and $u_{2}<u_{5}$ in $T$.) We either have $u_{1}<u_{2}$ or $u_{2}<u_{1}$ in $T$. Exploiting symmetry we may assume $u_{1}<u_{2}$ in $T$. (Indeed, if not then it suffices to exchange $\left(a_{1}, b_{1}\right)$ and $\left(a_{4}, b_{4}\right)$ with respectively $\left(a_{2}, b_{2}\right)$ and $\left(a_{3}, b_{3}\right)$.)

Applying Claim 27 on the three pairs $\left(a_{1}, b_{1}\right),\left(a_{4}, b_{4}\right),\left(a_{5}, b_{5}\right)$ and on the three pairs $\left(a_{2}, b_{2}\right),\left(a_{3}, b_{3}\right),\left(a_{5}, b_{5}\right)$, we obtain $y_{5} \leqslant b_{1}$ and $y_{2} \leqslant y_{5}$ in $P$. Since $\left(a_{1}, b_{1}\right),\left(a_{2}, b_{2}\right)$ is a 2-cycle and $u_{1}<u_{2}$ in $T$, we have $a_{1} \leqslant y_{2}$ in $P$ by Claim 25. But all together this implies $a_{1} \leqslant y_{2} \leqslant y_{5} \leqslant b_{1}$ in $P$, a contradiction.

Claim 30 The graph $J_{\Sigma, 1}$ is bipartite for every $\Sigma \in \Sigma\left(v_{13}\right)$. 


$$
\begin{aligned}
& \text { (i) }:=\left(a_{i}, b_{i}\right) \\
& \mathcal{E}=\{\{12,23\}, \\
& \{54,43\} \text {, }
\end{aligned}
$$
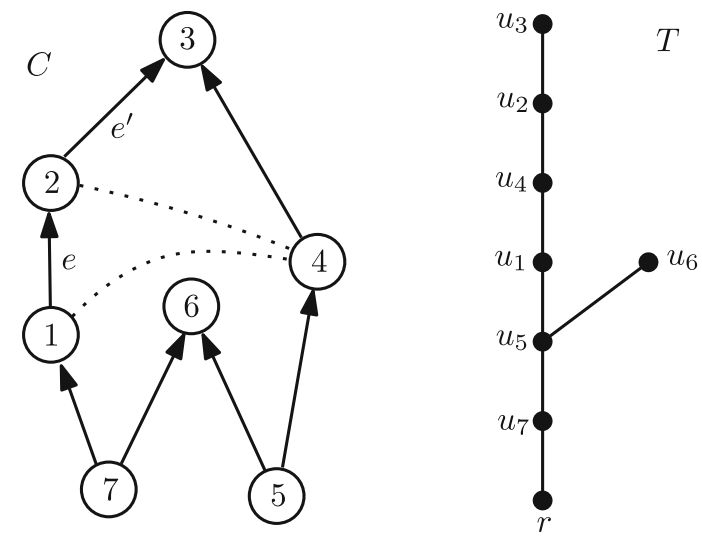

Fig. 10 Example for Claim 30: Cycle $C$ on the pairs $\left(a_{1}, b_{1}\right), \ldots,\left(a_{7}, b_{7}\right)$ and the positions of $u_{1}, \ldots, u_{7}$ in $T$. Given this, it follows that $u_{\{12,23\}}=u_{2}$ is maximal in $T$ among $u_{\{12,23\}}, u_{\{54,43\}}, u_{\{71,12\}}$. Following the proof for Claim 30 we would get that one of the dotted edges in the figure must be a real edge in $J_{\Sigma, 1}$, implying that $C$ cannot be induced

Proof Let $\Sigma \in \Sigma\left(v_{13}\right)$. Arguing by contradiction, suppose that $J_{\Sigma, 1}$ is not bipartite. Let $C$ be a shortest odd cycle in $J_{\Sigma, 1}$. Thus $C$ is induced, that is, $C$ has no chord. By Claim 26 we know that $C$ has length at least 5 .

We orient the edges of $J_{\Sigma, 1}$ in the following natural way: For each edge $\left\{(a, b),\left(a^{\prime}, b^{\prime}\right)\right\}$ in $J_{\Sigma, 1}$, we orient the edge towards $\left(a^{\prime}, b^{\prime}\right)$ if $u_{a b}<u_{a^{\prime} b^{\prime}}$ in $T$, and towards $(a, b)$ otherwise (that is, if $u_{a b}>u_{a^{\prime} b^{\prime}}$ in $T$ ). We encourage the reader to take a look at Fig. 10 for upcoming new notations and arguments.

Let $\mathcal{E}$ be the set of all pairs of consecutive edges in $C$ such that the source of one coincides with the target of the other. Since $C$ has an odd length, we have $|\mathcal{E}| \geqslant 1$. For every $\left\{e, e^{\prime}\right\} \in$ $\mathcal{E}$ consider the pair $(a, b) \in \operatorname{MM}\left(P, v_{13}, \Sigma\right)$ which is the common endpoint of $e$ and $e^{\prime}$ in $J_{\Sigma, 1}$, and let $u_{\left\{e, e^{\prime}\right\}}:=u_{a b}$.

Choose $\left\{e, e^{\prime}\right\} \in \mathcal{E}$ such that $u_{\left\{e, e^{\prime}\right\}}$ is maximal in $T$, that is, $u_{\left\{e, e^{\prime}\right\}} \nless u_{\left\{f, f^{\prime}\right\}}$ in $T$ for all $\left\{f, f^{\prime}\right\} \in \mathcal{E}$. Exchanging $e$ and $e^{\prime}$ if necessary we may assume that the target of $e$ coincides with the source of $e^{\prime}$. Enumerate the vertices of the odd cycle $C$ as $\left(a_{1}, b_{1}\right),\left(a_{2}, b_{2}\right), \ldots,\left(a_{k}, b_{k}\right)$ in such a way that $e=\left\{\left(a_{1}, b_{1}\right),\left(a_{2}, b_{2}\right)\right\}$ and $e^{\prime}=$ $\left\{\left(a_{2}, b_{2}\right),\left(a_{3}, b_{3}\right)\right\}$. Let $u_{i}:=u_{a_{i} b_{i}}, x_{i}:=x_{a_{i} b_{i}}, y_{i}:=y_{a_{i} b_{i}}$, and $z_{i}:=z_{a_{i} b_{i}}$ for each $i \in\{1,2, \ldots, k\}$. Thus $u_{1}<u_{2}<u_{3}$ in $T$ and $u_{2}=u_{\left\{e, e^{\prime}\right\}}$.

Let $i$ be the largest index in $\{3, \ldots, k\}$ such that $u_{2}<u_{j}$ for all $j \in\{3, \ldots, i\}$ in $T$. If there is an index $j \in\{3, \ldots, i\}$ such that $u_{j-1}<u_{j}<u_{j+1}$ or $u_{j-1}>u_{j}>u_{j+1}$ in $T$ (taking indices cyclically), then $u_{\left\{e, e^{\prime}\right\}}=u_{2}<u_{j}$ in $T$, which contradicts our choice of $\left\{e, e^{\prime}\right\}$ in $\mathcal{E}$ (since $\left\{\left\{\left(a_{j-1}, b_{j-1}\right),\left(a_{j}, b_{j}\right)\right\},\left\{\left(a_{j}, b_{j}\right),\left(a_{j+1}, b_{j+1}\right)\right\}\right\}$ was a better choice). Thus no such index $j$ exists. Given that $u_{2}<u_{3}$ in $T$, it follows that

$$
\left\{u_{j-1}, u_{j+1}\right\}<u_{j}
$$

in $T$ for each $o d d$ index $j \in\{3, \ldots, i\}$, and that $i$ is odd (because $u_{i+1}<u_{i}$ in $T$ by the choice of $i$ ).

Since $u_{2}<u_{i}$ and $u_{i+1}<u_{i}$ in $T$, the two nodes $u_{2}$ and $u_{i+1}$ are comparable in $T$. By our choice of $i$ we have $u_{2} \nless u_{i+1}$ in $T$. (This is clear if $i<k$, and if $i=k$ this follows from the fact that $u_{k+1}=u_{1}<u_{2}$ in $T$.) It follows that $u_{i+1} \leqslant u_{2}$ in $T$. We claim that

$$
u_{i+1}<u_{2}
$$


in $T$. So suppose $u_{i+1}=u_{2}$. Observe that $i \neq k$ (as otherwise $u_{i+1}=u_{1}<u_{2}$ in $T$ ) and $i \neq k-1$ (since $i$ and $k$ are odd) in this case. Thus, $3 \leqslant i \leqslant k-2$ and since the odd cycle $C$ is induced, it follows that the two pairs $\left(a_{1}, b_{1}\right),\left(a_{i+1}, b_{i+1}\right)$ do not form a 2-cycle. (For if they did, it would be a 2-cycle of type 1 since $u_{1}<u_{2}=$ $u_{i+1}$ in $T$, which would give a chord of $C$ in $J_{\Sigma, 1}$.) Applying Claim 27 on the pairs $\left(a_{1}, b_{1}\right),\left(a_{2}, b_{2}\right)$ and $\left(a_{i+1}, b_{i+1}\right)$ we obtain $a_{1} \leqslant x_{i+1}$ in $P$. Using Claim 25 on $\left(a_{1}, b_{1}\right)$ and $\left(a_{2}, b_{2}\right)$ gives us $x_{2} \leqslant b_{1}$ in $P$. However, since $u_{2}=u_{i+1}$ and $\left(\phi\left(x_{2}\right), \phi\left(y_{2}\right), \phi\left(z_{2}\right)\right)=$ $\left(\phi\left(x_{i+1}\right), \phi\left(y_{i+1}\right), \phi\left(z_{i+1}\right)\right)$ (given that $\left.\alpha_{11}\left(a_{2}, b_{2}\right)=\alpha_{11}\left(a_{i+1}, b_{i+1}\right)\right)$, we deduce $x_{2}=$ $x_{i+1}$, which implies $a_{1} \leqslant x_{i+1}=x_{2} \leqslant b_{1}$ in $P$, a contradiction. Therefore, $u_{i+1}<u_{2}$ in $T$, as claimed.

In order to finish the proof, we consider separately the case $i<k$ and $i=k$. First suppose that $i<k$, and thus $i \leqslant k-2$. Since $u_{i+1}<u_{2}<u_{i}$ and $u_{1}<u_{2}$ in $T$, using Claim 28 on the four pairs $\left(a_{i+1}, b_{i+1}\right),\left(a_{i}, b_{i}\right),\left(a_{1}, b_{1}\right),\left(a_{2}, b_{2}\right)$ we obtain that $\left\{\left(a_{i+1}, b_{i+1}\right),\left(a_{1}, b_{1}\right)\right\}$ or $\left\{\left(a_{i+1}, b_{i+1}\right),\left(a_{2}, b_{2}\right)\right\}$ is an edge in $J_{\Sigma, 1}$, showing that $C$ has a chord, a contradiction.

Next assume that $i=k$. Recall that $\left\{u_{j-1}, u_{j+1}\right\}<u_{j}$ in $T$ for each odd index $j \in\{3, \ldots, k\}$. It follows that $u_{j-1}$ and $u_{j+1}$ are comparable in $T$ for each such index $j$. Using Observation 8 and $k \geqslant 5$ we deduce in particular that there exists an even index $\ell \in\{4, \ldots, k-1\}$ such that $u_{\ell} \leqslant u_{\ell^{\prime}}$ for every even index $\ell^{\prime} \in\{4, \ldots, k-1\}$.

By the choice of $\ell$ we have $u_{\ell}<u_{3}$ in $T$ (since $u_{\ell} \leqslant u_{4}<u_{3}$ ) and $u_{\ell}<u_{k}$ in $T$ (since $u_{\ell} \leqslant u_{k-1}<u_{k}$ ). Note also that $u_{1}<u_{2}<u_{\ell}$ in $T$, since $i=k$. Applying Claim 29 on the five pairs $\left(a_{1}, b_{1}\right),\left(a_{2}, b_{2}\right),\left(a_{3}, b_{3}\right),\left(a_{k}, b_{k}\right)$ and $\left(a_{\ell}, b_{\ell}\right)$, we then obtain that $\left\{\left(a_{1}, b_{1}\right),\left(a_{\ell}, b_{\ell}\right)\right\}$ or $\left\{\left(a_{2}, b_{2}\right),\left(a_{\ell}, b_{\ell}\right)\right\}$ is an edge in $J_{\Sigma, 1}$, showing that $C$ has a chord, a contradiction.

Now that the bipartiteness of $J_{\Sigma, 1}$ is established for each $\Sigma \in \Sigma\left(v_{13}\right)$, to finish our proof of Lemma 22 (asserting that $J_{\Sigma}$ is 4-colorable), it remains to show that the two subgraphs of $J_{\Sigma, 2}$ induced by the two color classes in a 2-coloring of $J_{\Sigma, 1}$ are bipartite. Clearly, it is enough to show that every subgraph of $J_{\Sigma, 2}$ induced by an independent set of $J_{\Sigma, 1}$ is bipartite, which is exactly what we will do. (Recall that an independent set, also known as stable set, is a set of pairwise non-adjacent vertices.)

To this aim we introduce a new definition: Given a pair $(a, b) \in \operatorname{MM}(P)$ and a set $\{c, d\}$ of two elements of $P$, we say that $(a, b)$ is connected to $\{c, d\}$ if $a \leqslant c$ and $d \leqslant b$, or $a \leqslant d$ and $c \leqslant b$ in $P$. Note that if $(a, b)$ is connected to $\{c, d\}$, then it is in exactly one of two possible ways (that is, either $a \leqslant c$ and $d \leqslant b$, or $a \leqslant d$ and $c \leqslant b$ in $P$ ). Thus two pairs $(a, b),\left(a^{\prime}, b^{\prime}\right) \in \mathrm{MM}(P)$ connected to $\{c, d\}$ are either connected the same way, or in opposite ways. More generally, we can consider how a collection of pairs are connected to a certain set $\{c, d\}$, which we will need to do in what follows.

Observation 31 Let $\Sigma \in \Sigma\left(v_{13}\right)$. Let I be an independent set in $J_{\Sigma, 1}$ and let $c, d$ be two distinct elements of $P$. Suppose that $(a, b),\left(a^{\prime}, b^{\prime}\right) \in I$ are two pairs that are connected to $\{c, d\}$ in opposite ways. By definition $a \leqslant c \leqslant b^{\prime}$ and $a^{\prime} \leqslant d \leqslant b$, or $a \leqslant d \leqslant b^{\prime}$ and $a^{\prime} \leqslant c \leqslant b$ in $P$. Thus in both cases $(a, b),\left(a^{\prime}, b^{\prime}\right)$ is a 2-cycle, which must be of type 2 as $(a, b)$ and $\left(a^{\prime}, b^{\prime}\right)$ are non-adjacent in $J_{\Sigma, 1}$. In particular, $(a, b)$ and $\left(a^{\prime}, b^{\prime}\right)$ are adjacent in $J_{\Sigma, 2}$.

Observation 32 Let $\Sigma \in \Sigma\left(v_{13}\right)$. Let I be an independent set in $J_{\Sigma, 1}$ and let $c, d$ be two distinct elements of $P$. Suppose that $(a, b),\left(a^{\prime}, b^{\prime}\right) \in I$ are adjacent in $J_{\Sigma, 2}$ and that the two relations $a \leqslant b^{\prime}$ and $a^{\prime} \leqslant b$ both hit $\{c, d\}$. Then $(a, b),\left(a^{\prime}, b^{\prime}\right)$ are connected to $\{c, d\}$ in opposite ways. 
Claim 33 Let $\Sigma \in \Sigma\left(v_{13}\right)$. Let I be an independent set in $J_{\Sigma, 1}$ and let $c, d$ be two distinct elements of $P$. Suppose that $C$ is an induced odd cycle in the subgraph of $J_{\Sigma, 2}$ induced by I. If four of the pairs composing $C$ are connected to $\{c, d\}$ then they all are connected to $\{c, d\}$ the same way.

Proof Suppose that $\left(a_{1}, b_{1}\right), \ldots,\left(a_{4}, b_{4}\right)$ are four pairs from $C$ that are connected to $\{c, d\}$. (Note that these pairs are not necessarily consecutive in $C$.) If three of these pairs are connected to $\{c, d\}$ the same way and the fourth the other way, then by Observation 31 the fourth pair is adjacent to the first three in $J_{\Sigma, 2}$, which is not possible since the odd cycle $C$ is induced.

It follows that if $\left(a_{1}, b_{1}\right), \ldots,\left(a_{4}, b_{4}\right)$ are not connected to $\{c, d\}$ the same way, then without loss of generality $\left(a_{1}, b_{1}\right),\left(a_{2}, b_{2}\right)$ are connected to $\{c, d\}$ one way and $\left(a_{3}, b_{3}\right),\left(a_{4}, b_{4}\right)$ the other. We then deduce from Observation 31 that $\left(a_{1}, b_{1}\right),\left(a_{3}, b_{3}\right),\left(a_{2}, b_{2}\right),\left(a_{4}, b_{4}\right)$ is a cycle of length 4 in $J_{\Sigma, 2}$, a contradiction to the properties of $C$. Therefore, all four pairs must be connected to $\{c, d\}$ the same way.

Claim 34 Let $\Sigma \in \Sigma\left(v_{13}\right)$ and let I be an independent set in $J_{\Sigma, 1}$. Then the subgraph of $J_{\Sigma, 2}$ induced by $I$ is bipartite.

Proof Arguing by contradiction, suppose there is an odd cycle in the subgraph of $J_{\Sigma, 2}$ induced by $I$, and let $C$ be a shortest one. Enumerate the vertices of $C$ as $\left(a_{1}, b_{1}\right),\left(a_{2}, b_{2}\right), \ldots,\left(a_{k}, b_{k}\right)$ in order. Let $u_{i}:=u_{a_{i} b_{i}}$ and $s_{i}:=u_{i} \wedge u_{i+1}$ for each $i \in\{1, \ldots, k\}$ (cyclically). Recall that by the definition of $J_{\Sigma, 2}$ the pairs $\left(a_{i}, b_{i}\right)$ and $\left(a_{i+1}, b_{i+1}\right)$ form a 2-cycle of type 2 for each $i$, and thus $u_{i} \| u_{i+1}$ in $T$, implying that $s_{i}<\left\{u_{i}, u_{i+1}\right\}$ in $T$.

Let us start by pointing out the following consequence of Observation 32: If $i \in$ $\{1, \ldots, k\}$ and $\{c, d\}$ are such that the $u_{i}-u_{i+1}$ path in $T$ includes an edge $e$ of $T$ for which the intersection of the two bags of its endpoints is $\{c, d\}$, then $\left(a_{i}, b_{i}\right)$ and $\left(a_{i+1}, b_{i+1}\right)$ are connected to $\{c, d\}$ in opposite ways. This will be used a number of times in the proof.

Let $j \in\{1, \ldots, k\}$ be such that $s_{j}$ is maximal in $T$ among $s_{1}, \ldots, s_{k}$, that is, such that $s_{j} \nless s_{i}$ in $T$ for each $i \in\{1, \ldots, k\}$. Furthermore, if $s_{j} \neq u_{i}$ then we let $s_{j}^{i}$ be the neighbor of $s_{j}$ on the $s_{j}-u_{i}$ path in $T$, for each $i \in\{1, \ldots, k\}$. (Let us remark that we only make use of the notion $s_{j}^{i}$ when $s_{j} \neq u_{i}$ ). Thus in particular $s_{j}<\left\{s_{j}^{j}, s_{j}^{j+1}\right\}$ in $T$. See Fig. 11 for an illustration of this definition.

We claim that

$$
B\left(s_{j}\right) \cap B\left(s_{j}^{s}\right) \neq B\left(s_{j}\right) \cap B\left(s_{j}^{t}\right)
$$

for any $s, t \in\{1, \ldots, k\}$ with $s<t$ such that $s_{j}<\left\{s_{j}^{s}, s_{j}^{t}\right\}$ in $T$. Suppose to the contrary that $B\left(s_{j}\right) \cap B\left(s_{j}^{s}\right)=B\left(s_{j}\right) \cap B\left(s_{j}^{t}\right)=:\{c, d\}$. It follows from our choice of index $j$ that both the $u_{s-1}-u_{s}$ path and the $u_{s}-u_{s+1}$ path in $T$ include the edge $s_{j} s_{j}^{s}$ (otherwise $s_{j}<s_{s}$ in $T$ ), and similarly that the $u_{t}-u_{t+1}$ path in $T$ includes the edge $s_{j} s_{j}^{t}$ (otherwise $s_{j}<s_{t}$ in $T$ ). As a consequence we have that edge $s_{j} s_{j}^{s}$ is included in the $a_{s-1}^{T}-b_{s}^{T}$ path (as it passes through $u_{s-1}$ and $u_{s}$ ), the $a_{s}^{T}-b_{s-1}^{T}$ path, the $a_{s}^{T}-b_{s+1}^{T}$ path and the $a_{s+1}^{T}-b_{s}^{T}$ path, and similarly that edge $s_{j} s_{j}^{t}$ is included in the $a_{t}^{T}-b_{t+1}^{T}$ path and the $a_{t+1}^{T}-b_{t}^{T}$ path. Therefore, the pairs $\left(a_{s-1}, b_{s-1}\right),\left(a_{s}, b_{s}\right),\left(a_{s+1}, b_{s+1}\right),\left(a_{t}, b_{t}\right)$, and $\left(a_{t+1}, b_{t+1}\right)$ are all connected to $\{c, d\}$. Furthermore, $\left(a_{s-1}, b_{s-1}\right)$ and $\left(a_{s}, b_{s}\right)$ are connected in opposite ways, and the same holds for $\left(a_{s}, b_{s}\right)$ and $\left(a_{s+1}, b_{s+1}\right)$, as well as for $\left(a_{t}, b_{t}\right)$ and $\left(a_{t+1}, b_{t+1}\right)$. There cannot be four distinct pairs among these five, because otherwise this would contradict Claim 33. Hence the only possibility is that $k=3$ and $s=t-1$, and thus $s-1$ and $t+1$ are the same indices 
cyclically. But then recall that the $u_{1}-u_{2}$ path, the $u_{2}-u_{3}$ path and the $u_{3}-u_{1}$ path all have to use edge $s_{j} s_{j}^{s}$ or $s_{j} s_{j}^{t}$ in $T$. As a consequence, the three relations $a_{3} \leqslant b_{1}, a_{1} \leqslant b_{2}$ and $a_{2} \leqslant b_{3}$ all hit $\{c, d\}$. Hence two of them hit the same element, which implies $a_{i} \leqslant b_{i}$ for some $i \in\{1,2,3\}$. With this contradiction, we have proved $B\left(s_{j}\right) \cap B\left(s_{j}^{s}\right) \neq B\left(s_{j}\right) \cap B\left(s_{j}^{t}\right)$.

Since $B\left(s_{j}\right) \cap B\left(s_{j}^{i}\right)$ is a 2-element subset of $B\left(s_{j}\right)$ for each $i$ such that $s_{j} \neq u_{i}$, it directly follows that there are at most three indices $i$ such that $s_{j}<s_{j}^{i}$ in $T$ (recall that this inequality holds for $i=j$ and $i=j+1$ ).

This allows us to quickly dispense with the $k \geqslant 5$ case now: If $u_{j-1}>s_{j}$ in $T$ (and hence $s_{j}^{j-1}>s_{j}$ in $\left.T\right)$, then let $(s, t):=(j-1, j+1)$. Else, if $u_{j+2}>s_{j}$ in $T$, then let $(s, t):=(j, j+2)$. If neither of the two cases is true, we let $(s, t):=(j, j+1)$. We claim that in all three cases we obtain that

- $s_{j}<\left\{s_{j}^{s}, s_{j}^{t}\right\}$ in $T$,

- the two indices $s-1$ and $t+1$ are not the same (cyclically), and

- $s_{j}^{s-1}=s_{j}^{t+1}=\mathrm{p}\left(s_{j}\right)$.

The first two items are obvious, and the third one follows from our last observation and because $s_{j} \neq u_{s-1}$ and $s_{j} \neq u_{t+1}$ (recall that $u_{s-1} \| u_{s}$ and $u_{t} \| u_{t+1}$ in $T$ ).

Then, the $u_{s-1}-u_{s}$ path and the $u_{t}-u_{t+1}$ path in $T$ both include the edge $\mathrm{p}\left(s_{j}\right) s_{j}$. It follows that $\left(a_{s-1}, b_{s-1}\right)$ and $\left(a_{s}, b_{s}\right)$ are connected to $B\left(s_{j}\right) \cap B\left(\mathrm{p}\left(s_{j}\right)\right)$ in opposite ways, and that the same holds for $\left(a_{t}, b_{t}\right)$ and $\left(a_{t+1}, b_{t+1}\right)$. Since these four pairs are distinct, this contradicts Claim 33 and concludes the case $k \geqslant 5$.

It remains to consider the $k=3$ case. Reordering the pairs of $C$ if necessary we may assume $j=1$ and $B\left(s_{1}\right)=\{c, d, e\}$ with $B\left(s_{1}\right) \cap B\left(s_{1}^{1}\right)=\{c, d\}$ and $B\left(s_{1}\right) \cap B\left(s_{1}^{2}\right)=\{d, e\}$. The two relations $a_{1} \leqslant b_{2}$ and $a_{2} \leqslant b_{1}$ both hit $\{c, d\}$ and $\{d, e\}$, and clearly they cannot hit the same element. Thus, one of the relations hits $d$, and the other $c$ and $e$. Exploiting symmetry again, we may assume without loss of generality that $a_{2} \leqslant b_{1}$ hits $d$. (Indeed, if not then this can be achieved by reversing the ordering of the pairs of $C$.) Thus we have

$$
a_{2} \leqslant d \leqslant b_{1}
$$

in $P$, which then implies

$$
a_{1} \leqslant c \leqslant e \leqslant b_{2}
$$

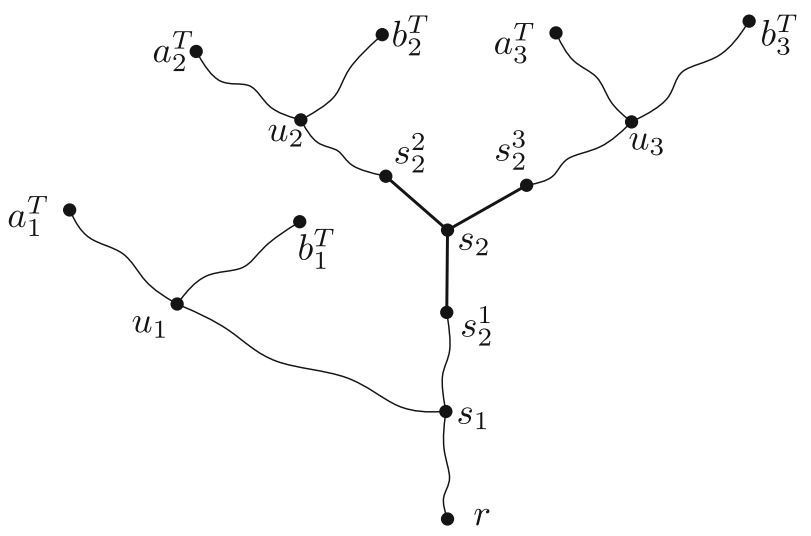

Fig. 11 Illustration for Claim 34 with $j=2$ 
in $P$ by Observation 10. Now, the two relations $a_{2} \leqslant b_{3}$ and $a_{3} \leqslant b_{1}$ both hit $B\left(s_{1}\right)=$ $\{c, d, e\}$. (Here we use that $s_{1} \nless\left\{s_{2}, s_{3}\right\}$ in $T$.) Neither hit $c$ or $e$ since this would contradict $a_{1} \leqslant c \leqslant e \leqslant b_{2}$ in $P$. Hence both relations hit $d$, which implies $a_{3} \leqslant d \leqslant b_{3}$ in $P$, a contradiction.

This concludes the proof of Lemma 22, asserting that $J_{\Sigma}$ is 4-colorable for each $\Sigma \in$ $\Sigma\left(v_{13}\right)$. Now, for each $\Sigma \in \Sigma\left(v_{13}\right)$ let $\psi_{13, \Sigma}$ be such a coloring. Then we define $\alpha_{13}$ as follows:

For each $\Sigma \in \boldsymbol{\Sigma}\left(\nu_{13}\right)$ and pair $(a, b) \in \operatorname{MM}\left(P, \nu_{13}, \Sigma\right)$, we let

$$
\alpha_{13}(a, b):=\psi_{13, \Sigma}(a, b) \text {. }
$$

\subsection{Node $v_{14}$ and its function $\alpha_{14}$ : Dealing with strict alternating cycles of length at least 3}

Recall that compared to $\operatorname{MM}\left(P, v_{14}, \Sigma\right)$, pairs in $\operatorname{MM}\left(P, v_{14}, \Sigma\right)$ have the additional property that they do not form a 2-cycle with another pair of $\operatorname{MM}\left(P, v_{14}, \Sigma\right)$, thanks to function $\alpha_{13}$. Therefore, strict alternating cycles in $\operatorname{MM}\left(P, v_{14}, \Sigma\right)\left(\Sigma \in \Sigma\left(v_{14}\right)\right)$ have length at least 3 . We will now list (and prove) a number of properties satisfied by these alternating cycles. First we prove a claim that bears some similarity with Claim 12 .

Claim 35 Let $\Sigma \in \Sigma\left(v_{14}\right)$ and suppose that $\left\{\left(a_{i}, b_{i}\right)\right\}_{i=1}^{k}$ is a strict alternating cycle in $\operatorname{MM}\left(P, v_{14}, \Sigma\right)$. Let $u_{i}$ denote $u_{a_{i} b_{i}}$ for each $i \in\{1,2, \ldots, k\}$. Then there is an index $j \in\{1,2, \ldots, k\}$ such that $u_{j} \leqslant u_{i}$ in $T$ for each $i \in\{1,2, \ldots, k\}$.

Proof We denote $w_{a_{i} b_{i}}, p_{a_{i} b_{i}}, x_{a_{i} b_{i}}, y_{a_{i} b_{i}}, z_{a_{i} b_{i}}$ by $w_{i}, p_{i}, x_{i}, y_{i}, z_{i}$ respectively, for each $i \in\{1,2, \ldots, k\}$. We may assume $\alpha_{1}\left(a_{i}, b_{i}\right)=$ left.

Consider the nodes $u_{1}, \ldots, u_{k}$ and let $j \in\{1,2, \ldots, k\}$ be such that $u_{j}$ is minimal in $T$ among these. We will show that $u_{j} \leqslant u_{i}$ in $T$ for each $i \in\{1,2, \ldots, k\}$. This can equivalently be rephrased as follows: Every element $u_{i}$ which is minimal in $T$ among $u_{1}, \ldots, u_{k}$ satisfies $u_{i}=u_{j}$ (note that we could possibly have $u_{i}=u_{j}$ for $i \neq j$ ). Arguing by contradiction, let us assume that there is an element minimal in $T$ among $u_{1}, \ldots, u_{k}$ which is distinct from $u_{j}$.

We start by showing that under this assumption $u_{1}, \ldots, u_{k}$ are all pairwise incomparable in $T$ (and thus are in particular all distinct). Once this is established, we will then be able to derive the desired contradiction.

Of course, to prove that $u_{1}, \ldots, u_{k}$ are pairwise incomparable in $T$ it is enough to show that $u_{i} \| u_{j}$ in $T$ for each $i \in\{1,2, \ldots, k\}$ with $i \neq j$, since $u_{j}$ was chosen as an arbitrary minimal element in $T$ among $u_{1}, \ldots, u_{k}$. Assume not, that is, that there is an index $i \in$ $\{1,2, \ldots, k\}$ with $i \neq j$ such that $u_{j} \leqslant u_{i}$ in $T$. We may choose $i$ in such a way that we additionally have $u_{i-1} \| u_{j}$ or $u_{i+1} \| u_{j}$ in $T$. As the arguments for the two cases are analogous we consider only the case $u_{i-1} \| u_{j}$ in $T$.

We have $a_{i-1}^{T} \ngtr u_{j}$ since $u_{i-1} \| u_{j}$ in $T$, and we also have $u_{j} \leqslant u_{i}<b_{i}^{T}$ in $T$. It follows that the path from $a_{i-1}^{T}$ to $b_{i}^{T}$ in $T$ goes through the edge $p_{j} u_{j}$. Thus, the relation $a_{i-1} \leqslant b_{i}$ hits $B\left(p_{j}\right) \cap B\left(u_{j}\right)=\left\{x_{j}, y_{j}\right\}$. But then $a_{i-1} \leqslant x_{j} \leqslant b_{i}$ or $a_{i-1} \leqslant y_{j} \leqslant$ $b_{i}$ in $P$, which implies $a_{j} \leqslant x_{j} \leqslant b_{i}$ or $a_{i-1} \leqslant y_{j} \leqslant b_{j}$. Since we have $i \neq j+1$ 
(as $u_{i-1} \| u_{j}$ in $T$ ) and $j \neq i$, this contradicts the assumption that our alternating cycle $\left(a_{1}, b_{1}\right),\left(a_{2}, b_{2}\right), \ldots,\left(a_{k}, b_{k}\right)$ is strict.

We conclude that $u_{1}, \ldots, u_{k}$ are all pairwise incomparable in $T$, as claimed.

Let $s_{i}:=u_{i} \wedge u_{i+1}$ for each $i \in\{1,2, \ldots, k\}$ (indices are taken cyclically, as always). Note that the path from $a_{i}^{T}$ to $b_{i+1}^{T}$ in $T$ has to go through $s_{i}$. Choose $i \in\{1, \ldots, k\}$ such that $s_{i}$ is maximal among $s_{1}, \ldots, s_{k}$ in $T$. The nodes $s_{i-1}$ and $s_{i}$ are comparable in $T$, since $s_{i-1} \leqslant u_{i}$ and $s_{i} \leqslant u_{i}$ in $T$. Thus we have $s_{i-1} \leqslant s_{i}$ in $T$. Similarly, $s_{i+1} \leqslant s_{i}$ in $T$.

Let us first look at the case $s_{i-1}=s_{i}$. This implies $s_{i} \leqslant\left\{u_{i-1}, u_{i}, u_{i+1}\right\}$ in $T$. Now the $a_{i-1}^{T}-b_{i}^{T}$ path, the $a_{i}^{T}-b_{i+1}^{T}$ path, and the $a_{i+1}^{T}-b_{i+2}^{T}$ path in $T$ all go through $s_{i}$ in $T$. This means that the relations $a_{i-1} \leqslant b_{i}, a_{i} \leqslant b_{i+1}$ and $a_{i+1} \leqslant b_{i+2}$ all hit $B\left(s_{i}\right)$. Clearly, no two of them can hit the same element (recall that $k \geqslant 3$ and that our alternating cycle is strict), and hence each element of $B\left(s_{i}\right)$ is hit by exactly one of these three relations. On the other hand, the three paths from $r$ to $b_{i-1}^{T}, b_{i}^{T}$ and $b_{i+1}^{T}$ in $T$ all go through $\mathrm{p}\left(s_{i}\right)$ and $s_{i}$, implying that the relations $a_{0} \leqslant b_{i-1}, a_{0} \leqslant b_{i}$, and $a_{0} \leqslant b_{i+1}$ all hit $B\left(\mathrm{p}\left(s_{i}\right)\right) \cap B\left(s_{i}\right)$. In particular, some element in $B\left(s_{i}\right)$ is hit by at least two of these three relations. But with the observations made before, it follows that some element in $\left\{a_{i-1}, a_{i}, a_{i+1}\right\}$ is below two elements of $\left\{b_{i-1}, b_{i}, b_{i+1}\right\}$ in $P$, which is not possible in a strict alternating cycle. Therefore, $s_{i-1} \neq s_{i}$.

Thus we have $s_{i-1}<s_{i}$ in $T$, and with a similar argument one also deduces that $s_{i+1}<s_{i}$ in $T$.

To conclude the proof, consider the $a_{i-1}^{T}-b_{i}^{T}$ path, the $a_{i+1}^{T}-b_{i+2}^{T}$ path, and the $r-b_{i+1}^{T}$ path in $T$. They all go through the edge $\mathrm{p}\left(s_{i}\right) s_{i}$ of $T$, and hence the corresponding relations in $P$ all hit $B\left(\mathrm{p}\left(s_{i}\right)\right) \cap B\left(s_{i}\right)$. Therefore, two of these relations hit the same element in that set, which again contradicts the fact that our alternating cycle is strict.

By Claim 35 we are in a situation similar to that first encountered in Section 3.5, namely for each $\Sigma \in \Sigma\left(v_{14}\right)$ each alternating cycle in $\operatorname{MM}\left(P, v_{14}, \Sigma\right)$ can be written as $\left\{\left(a_{i}, b_{i}\right)\right\}_{i=1}^{k}$ in such a way that $u_{a_{1} b_{1}} \leqslant u_{a_{i} b_{i}}$ in $T$ for each $i \in\{1, \ldots, k\}$. We may further assume that the pair $\left(a_{1}, b_{1}\right)$ is such that $b_{1}^{T}$ is to the right of $b_{i}^{T}$ in $T$ if $a_{1}^{T}$ is to the left of $b_{1}^{T}$ in $T$, and to the left of $b_{i}^{T}$ otherwise, for each $i \in\{2, \ldots, k\}$ such that $u_{a_{1} b_{1}}=u_{a_{i} b_{i}}$. As before the pair $\left(a_{1}, b_{1}\right)$ is uniquely defined, and we call it the root of the alternating cycle.

Our next claim mirrors Claim 14 from Section 3.5.

Claim 36 Let $\Sigma \in \Sigma\left(v_{14}\right)$ and suppose that $\left\{\left(a_{i}, b_{i}\right)\right\}_{i=1}^{k}$ is a strict alternating cycle in $\operatorname{MM}\left(P, v_{14}, \Sigma\right)$ with root $\left(a_{1}, b_{1}\right)$. Let $u_{i}, w_{i}$ denote $u_{a_{i} b_{i}}, w_{a_{i} b_{i}}$ respectively, for each $i \in$ $\{1,2, \ldots, k\}$. Then $u_{1}<w_{1} \leqslant u_{i}$ in $T$ for each $i \in\{2, \ldots, k\}$.

Proof We denote $p_{a_{i} b_{i}}, x_{a_{i} b_{i}}, y_{a_{i} b_{i}}, z_{a_{i} b_{i}}$ by $p_{i}, x_{i}, y_{i}, z_{i}$ respectively, for each $i \in$ $\{1,2, \ldots, k\}$. We may assume $\alpha_{1}\left(a_{i}, b_{i}\right)=$ left for each $i \in\{1,2, \ldots, k\}$.

First we will show that $u_{1}<w_{1} \leqslant u_{k}$ in $T$. To do so suppose first that $u_{1}=u_{k}$. Then $w_{1} \nless a_{k}^{T}$ in $T$, as otherwise we would have $b_{k}^{T}$ to the right of $b_{1}^{T}$ in $T$ (Observation 9), which contradicts the choice of $\left(a_{1}, b_{1}\right)$ as the root of the strict alternating cycle. In particular, the path from $a_{k}^{T}$ to $b_{1}^{T}$ in $T$ goes through $u_{1}$. Hence the relation $a_{k} \leqslant b_{1}$ hits $B\left(u_{1}\right)=\left\{x_{1}, y_{1}, z_{1}\right\}$; let $q \in B\left(u_{1}\right)$ be such that $a_{k} \leqslant q \leqslant b_{1}$ in $P$. Clearly, $q \in\left\{y_{1}, z_{1}\right\}$. Given that $u_{1}=u_{k}$ and $\left(\phi\left(x_{1}\right), \phi\left(y_{1}\right), \phi\left(z_{1}\right)\right)=\left(\phi\left(x_{k}\right), \phi\left(y_{k}\right), \phi\left(z_{k}\right)\right)$ (since $\alpha_{11}\left(a_{1}, b_{1}\right)=\alpha_{11}\left(a_{k}, b_{k}\right)$ ), we obviously have $x_{1}=x_{k}, y_{1}=y_{k}$, and $z_{1}=z_{k}$. If $q=y_{1}=y_{k}$ then we directly obtain $q \leqslant b_{k}$ in $P$. If $q=z_{1}=z_{k}$ then we also deduce $q \leqslant b_{k}$ in $P$, because $\alpha_{12}\left(a_{1}, b_{1}\right)=\alpha_{12}\left(a_{k}, b_{k}\right)$, and thus in particular $z_{k} \leqslant b_{k}$ in $P$ since $z_{1} \leqslant b_{1}$. Hence in both cases $q \leqslant b_{k}$ in $P$. This implies $a_{k} \leqslant q \leqslant b_{k}$ in $P$, a contradiction. Therefore, $u_{1} \neq u_{k}$, and $u_{1}<u_{k}$ in $T$. 
Let $w^{\prime}$ be the neighbor of $u_{1}$ on the $u_{1}-u_{k}$ path in $T$. In order to show $u_{1}<w_{1} \leqslant u_{k}$ in $T$, it remains to prove $w^{\prime}=w_{1}$. Suppose to the contrary that $w^{\prime} \neq w_{1}$. Then the $a_{k}^{T}-b_{1}^{T}$ path and the $r-b_{k}^{T}$ path in $T$ both go through $u_{1}$ and $w^{\prime}$. Hence the relations $a_{k} \leqslant b_{1}$ and $a_{0} \leqslant b_{k}$ both hit $B\left(u_{1}\right) \cap B\left(w^{\prime}\right) \varsubsetneqq\left\{x_{1}, y_{1}, z_{1}\right\}$. Clearly, they cannot hit the same element. None of the two relations hit $x_{1}$, as otherwise $a_{1} \leqslant x_{1} \leqslant b_{1}$ or $a_{1} \leqslant x_{1} \leqslant b_{k}$ in $P$ (which is not possible since $k \geqslant 3$ and the alternating cycle is strict). We conclude that $B\left(u_{1}\right) \cap B\left(w^{\prime}\right)=\left\{y_{1}, z_{1}\right\}$. Since the relation $a_{0} \leqslant b_{k}$ also hits $B\left(u_{1}\right) \cap B\left(p_{1}\right)=\left\{x_{1}, y_{1}\right\}$, and thus hits $y_{1}$, it follows that $a_{0} \leqslant y_{1} \leqslant b_{k}$ and $a_{k} \leqslant z_{1} \leqslant b_{1}$ in $P$. Now let $i \in\{1, \ldots, k-1\}$ be maximal such that $w^{\prime} \nless u_{i}$ in $T$. Note that there is such an index since $w^{\prime} \nless u_{1}$ in $T$. If $w^{\prime} \leqslant a_{i}^{T}$ in $T$, then the path from $a_{i}^{T}$ to $u_{i}$ in $T$ goes through the edge $u_{1} w^{\prime}$. If, on the other hand, $w^{\prime} \| a_{i}^{T}$ in $T$, then the path from $a_{i}^{T}$ to $b_{i+1}^{T}$ in $T$ goes through the edge $u_{1} w^{\prime}$. Thus at least one of $a_{i} \leqslant x_{i}$ and $a_{i} \leqslant b_{i+1}$ hits $B\left(u_{1}\right) \cap B\left(w^{\prime}\right)=\left\{y_{1}, z_{1}\right\}$. Hence $a_{i} \leqslant y_{1}$ or $a_{i} \leqslant z_{1}$ in $P$. However, since $\left\{y_{1}, z_{1}\right\} \leqslant b_{1}$ in $P$, this implies $a_{i} \leqslant b_{1}$ in both cases. Given that $i<k$, this contradicts the fact that the alternating cycle is strict. Therefore, we must have $w^{\prime}=w_{1}$, and $u_{1}<w_{1} \leqslant u_{k}$ in $T$, as claimed.

So far we know that $w_{1} \leqslant u_{i}$ in $T$ for $i=k$, and it remains to show it for each $i \in\{2, \ldots, k-1\}$. Arguing by contradiction, assume that this does not hold, and let $i \in\{2, \ldots, k-1\}$ be maximal such that $w_{1} \nless u_{i}$ in $T$. By our choice it holds that $w_{1} \leqslant u_{i+1}$ in $T$, even in the case $i=k-1$.

First suppose that $u_{i}=u_{1}$. Then $w_{1} \nless a_{i}^{T}$ in $T$, because otherwise $b_{i}^{T}$ would be to the right of $b_{1}^{T}$ in $T$ (Observation 9), contradicting the fact that $\left(a_{1}, b_{1}\right)$ is the root of the strict alternating cycle. But then, the path from $a_{i}^{T}$ to $b_{i+1}^{T}$ goes through the edge $u_{1} w_{1}$ (since $w_{1} \leqslant u_{i+1}$ in $\left.T\right)$. Thus the relation $a_{i} \leqslant b_{i+1}$ hits in particular $B\left(u_{1}\right)$; let $q \in B\left(u_{1}\right)$ be such that $a_{i} \leqslant q \leqslant b_{i+1}$ in $P$. Given that $u_{1}=u_{i}$ we deduce $x_{1}=x_{i}, y_{1}=y_{i}$, and $z_{1}=z_{i}$ (using $\left.\alpha_{11}\left(a_{1}, b_{1}\right)=\alpha_{11}\left(a_{i}, b_{i}\right)\right)$, and hence $a_{1} \leqslant q$ in $P\left(\right.$ using $\left.\alpha_{12}\left(a_{1}, b_{1}\right)=\alpha_{12}\left(a_{i}, b_{i}\right)\right)$, exactly as in the beginning of the proof. This implies $a_{1} \leqslant q \leqslant b_{i+1}$ in $P$, and as $i+1 \geqslant 3$ this contradicts once again the fact that the alternating cycle is strict. Therefore, $u_{1} \neq u_{i}$, and $u_{1}<u_{i}$ in $T$.

Let $w^{\prime}$ be the neighbor of $u_{1}$ on the $u_{1}-u_{i}$ path in $T$. Note that $w^{\prime} \neq w_{1}$. The $a_{i}^{T}-b_{i+1}^{T}$ path and the $r-b_{i}^{T}$ path both go through the edge $u_{1} w^{\prime}$. Thus the relations $a_{i} \leqslant b_{i+1}$ and $a_{0} \leqslant b_{i}$ both hit $B\left(u_{1}\right) \cap B\left(w^{\prime}\right) \varsubsetneqq\left\{x_{1}, y_{1}, z_{1}\right\}$. Clearly, they cannot hit the same element. Since $a_{i} \leqslant x_{1} \leqslant b_{i+1}$ in $P$ would imply $a_{1} \leqslant b_{i+1}$ (which is not possible since $i+1 \geqslant 3$ ) while $a_{i} \leqslant y_{1} \leqslant b_{i+1}$ would imply $a_{i} \leqslant b_{1}$ (which cannot be since $i<k$ ), we deduce

$$
a_{i} \leqslant z_{1} \leqslant b_{i+1}
$$

in $P$, and

$$
a_{0} \leqslant q \leqslant b_{i}
$$

in $P$, where $q$ is the element in $\left\{x_{1}, y_{1}\right\}$ such that $B\left(u_{1}\right) \cap B\left(w^{\prime}\right)=\left\{q, z_{1}\right\}$.

We distinguish two cases, depending whether $q=x_{1}$ or $q=y_{1}$. First suppose that $q=x_{1}$. Since $a_{0} \leqslant q=x_{1} \leqslant b_{i}$ in $P$, this implies $a_{1} \leqslant x_{1} \leqslant b_{i}$ in $P$, and hence $i=2$ (otherwise, the alternating cycle would not be strict). Furthermore, given that $a_{0} \leqslant x_{1}$ in $P$ and $\alpha_{12}\left(a_{1}, b_{1}\right)=\alpha_{12}\left(a_{2}, b_{2}\right)$, we have $a_{0} \leqslant x_{2}$ in $P$ as well. The $r-u_{2}$ path in $T$ includes the edge $u_{1} w^{\prime}$ since $w^{\prime} \leqslant u_{2}$ in $T$. Using that $x_{2} \in B\left(u_{2}\right)$, we deduce that the relation $a_{0} \leqslant x_{2}$ in $P$ hits $B\left(u_{1}\right) \cap B\left(w^{\prime}\right)=\left\{x_{1}, z_{1}\right\}$. In particular, at least one of $x_{1} \leqslant x_{2}$ and $z_{1} \leqslant x_{2}$ holds in $P$. Before considering each of these two possibilities, let us observe that the $a_{2}^{T}-u_{1}$ path in $T$ includes the edge $u_{2} p_{2}$. It follows that the relation $a_{2} \leqslant z_{1}$ hits $B\left(u_{2}\right) \cap B\left(p_{2}\right)=\left\{x_{2}, y_{2}\right\}$. Clearly, it cannot hit $y_{2}$ (otherwise $a_{2} \leqslant y_{2} \leqslant b_{2}$ ), and hence $x_{2} \leqslant z_{1}$ in $P$. 
Now, if $z_{1} \leqslant x_{2}$ in $P$ then $x_{2}=z_{1}$. However, we also know that $\phi\left(x_{2}\right)=\phi\left(x_{1}\right) \neq \phi\left(z_{1}\right)$, since $\alpha_{11}\left(a_{1}, b_{1}\right)=\alpha_{11}\left(a_{2}, b_{2}\right)$, which is a contradiction.

On the other hand, if $x_{1} \leqslant x_{2}$ in $P$ then $a_{1} \leqslant x_{1} \leqslant x_{2} \leqslant z_{1} \leqslant b_{i+1}=b_{3}$ in $P$, which contradicts the fact the alternating cycle is strict. This concludes the case where $q=x_{1}$.

Next, assume $q=y_{1}$. Let $j \in\{1, \ldots, i-1\}$ be maximal such that $w^{\prime} \nless u_{j}$ in $T$. (Note that there is such an index $j$ since $w^{\prime} \nless u_{1}$ in $T$.) If $w^{\prime} \leqslant a_{j}^{T}$ in $T$ then the path from $a_{j}^{T}$ to $u_{j}$ in $T$ goes through the edge $u_{1} w^{\prime}$. If, on the other hand, $w^{\prime} \| a_{j}^{T}$ in $T$, then the path from $a_{j}^{T}$ to $b_{j+1}^{T}$ in $T$ goes through the edge $u_{1} w^{\prime}$ since $w^{\prime} \leqslant u_{j+1}<b_{j+1}^{T}$ in $T$. Hence at least one of the two relations $a_{j} \leqslant x_{j}$ and $a_{j} \leqslant b_{j+1}$ hits $\left\{q, z_{1}\right\}=\left\{y_{1}, z_{1}\right\}$. It follows that $a_{j} \leqslant y_{1}$ or $a_{j} \leqslant z_{1}$ in $P$. The first inequality implies $a_{j} \leqslant y_{1} \leqslant b_{1}$ in $P$, a contradiction since $j \neq k$. The second inequality implies $a_{j} \leqslant z_{1} \leqslant b_{i+1}$ in $P$, which is not possible since $j \neq i$. This concludes the proof.

Claim 37 Let $\Sigma \in \Sigma\left(v_{14}\right)$ and suppose that $\left\{\left(a_{i}, b_{i}\right)\right\}_{i=1}^{k}$ is a strict alternating cycle in $\operatorname{MM}\left(P, v_{14}, \Sigma\right)$ with root $\left(a_{1}, b_{1}\right)$. Let $u_{i}$ denote $u_{a_{i} b_{i}}$ for each $i \in\{1,2, \ldots, k\}$. Then the $u_{1}-b_{1}^{T}$ path in $T$ avoids $u_{2}$.

Proof We denote $w_{a_{i} b_{i}}, p_{a_{i} b_{i}}, x_{a_{i} b_{i}}, y_{a_{i} b_{i}}$, by $w_{i}, p_{i}, x_{i}, y_{i}$ respectively, for each $i \in$ $\{1,2, \ldots, k\}$. We may assume $\alpha_{1}\left(a_{i}, b_{i}\right)=$ left.

Arguing by contradiction, suppose that $u_{1} \leqslant u_{2}<b_{1}^{T}$ in $T$. By Claim 36 we know $u_{1}<w_{1} \leqslant u_{2}<b_{2}^{T}$ in $T$. The $a_{1}^{T}-b_{2}^{T}$ path in $T$ goes through the edge $p_{2} u_{2}$. Hence the relation $a_{1} \leqslant b_{2}$ hits $B\left(p_{2}\right) \cap B\left(u_{2}\right)=\left\{x_{2}, y_{2}\right\}$. Clearly, it cannot hit $x_{2}$ because otherwise $a_{2} \leqslant x_{2} \leqslant b_{2}$ in $P$. Therefore, $a_{1} \leqslant y_{2} \leqslant b_{2}$ in $P$.

Now consider the path connecting $r$ to $b_{1}^{T}$ in $T$. This path also includes the edge $p_{2} u_{2}$. Thus the relation $a_{0} \leqslant b_{1}$ hits $\left\{x_{2}, y_{2}\right\}$. If it hits $x_{2}$, then we obtain $a_{2} \leqslant x_{2} \leqslant b_{1}$ in $P$, which contradicts the fact that the alternating cycle is strict (recall that $k \geqslant 3$ ). If it hits $y_{2}$, then we deduce $a_{1} \leqslant y_{2} \leqslant b_{1}$ in $P$, again a contradiction.

Let $\Sigma \in \Sigma\left(v_{14}\right)$ and suppose that $\left\{\left(a_{i}, b_{i}\right)\right\}_{i=1}^{k}$ is a strict alternating cycle in $\operatorname{MM}\left(P, v_{14}, \Sigma\right)$ with root $\left(a_{1}, b_{1}\right)$. In what follows we will need to consider the nodes $q_{i}:=u_{i} \wedge b_{1}^{T}$ of $T$ where $i \in\{1,2, \ldots, k\}$. Observe that

$$
u_{1}<w_{1} \leqslant q_{i} \leqslant u_{i}
$$

in $T$ for each $i \in\{2,3, \ldots, k\}$ by Claim 36 , and

$$
q_{2}<u_{2}
$$

in $T$ by Claim 37.

Claim 38 Let $\Sigma \in \Sigma\left(v_{14}\right)$ and suppose that $\left\{\left(a_{i}, b_{i}\right)\right\}_{i=1}^{k}$ is a strict alternating cycle in $\operatorname{MM}\left(P, v_{14}, \Sigma\right)$ with root $\left(a_{1}, b_{1}\right)$. Let $u_{i}$ denote $u_{a_{i} b_{i}}$ and let $q_{i}:=u_{i} \wedge b_{1}^{T}$, for each $i \in\{1,2, \ldots, k\}$. Then

(i) $u_{i} \nless q_{2}$ in $T$ for each $i \in\{3,4, \ldots, k\}$, and

(ii) $u_{1}<q_{2} \leqslant q_{3}<b_{1}^{T}$ in $T$.

Proof We denote $w_{a_{i} b_{i}}, p_{a_{i} b_{i}}, x_{a_{i} b_{i}}, y_{a_{i} b_{i}}$, by $w_{i}, p_{i}, x_{i}, y_{i}$ respectively, for each $i \in$ $\{1,2, \ldots, k\}$. We may assume $\alpha_{1}\left(a_{i}, b_{i}\right)=$ left. 
To prove (i) we argue by contradiction: Suppose $u_{i} \leqslant q_{2}$ in $T$ for some $i \in\{3,4, \ldots, k\}$. Since $q_{2}<b_{1}^{T}$ in $T$, and $u_{1}<w_{1} \leqslant u_{i}$ by Claim 36, it follows that $u_{1}<w_{1} \leqslant u_{i} \leqslant$ $q_{2}<b_{1}^{T}$ in $T$. In particular, the path connecting $a_{1}^{T}$ to $b_{2}^{T}$ in $T$ goes through the edge $p_{i} u_{i}$. Hence the relation $a_{1} \leqslant b_{2}$ hits $B\left(p_{i}\right) \cap B\left(u_{i}\right)=\left\{x_{i}, y_{i}\right\}$. If it hits $x_{i}$ then $a_{i} \leqslant x_{i} \leqslant b_{2}$ in $P$, while if it hits $y_{i}$ then $a_{1} \leqslant y_{i} \leqslant b_{i}$ in $P$. In both cases it contradicts the fact that the alternating cycle is strict.

Let us now prove (ii). Using Claim 36 we already deduce that $u_{1}<\left\{q_{2}, q_{3}\right\}<b_{1}^{T}$ in $T$. Thus, it remains to show $q_{2} \leqslant q_{3}$ in $T$. Arguing by contradiction, suppose $q_{3}<q_{2}$ in $T$ (note that $q_{2}$ and $q_{3}$ are comparable in $T$ ). Let $i$ be the largest index in $\{3,4, \ldots, k\}$ such that $q_{i}<q_{2}$ in $T$. If $i<k$ then $q_{i}<q_{2} \leqslant q_{i+1} \leqslant u_{i+1}<b_{i+1}^{T}$ in $T$. If $i=k$ then clearly $q_{i}<q_{2}<b_{1}^{T}$ in $T$. Thus in both cases

$$
q_{i}<q_{2}<b_{i+1}^{T}
$$

in $T$ (taking indices cyclically).

Observe also that

$$
q_{2} \nless a_{i}^{T}
$$

in $T$. Indeed, if $q_{2} \leqslant a_{i}^{T}$ in $T$ then $q_{2} \leqslant b_{i}^{T}$ as well, since otherwise $u_{i}<q_{2}$ in $T$, contradicting (i). However, this implies $q_{2} \leqslant u_{i}$ in $T$, and hence $q_{2} \leqslant q_{i}$ since $q_{2}<b_{1}^{T}$, a contradiction.

Now consider the edge $\mathrm{p}\left(q_{2}\right) q_{2}$ in $T$ and let $B\left(\mathrm{p}\left(q_{2}\right)\right) \cap B\left(q_{2}\right)=\{c, d\}$. In the following, we aim to show that the relevant part of $T$ essentially looks like in Fig. 12, and consequently that the relations $a_{i} \leqslant b_{i+1}$ and $a_{2} \leqslant b_{3}$ have to hit $\{c, d\}$. From this observation we will obtain our final contradiction.

Using (23) and that $q_{2} \leqslant b_{i+1}^{T}$ in $T$ (see (22)), we deduce that the path from $a_{i}^{T}$ to $b_{i+1}^{T}$ in $T$ goes through this edge. Thus the relation $a_{i} \leqslant b_{i+1}$ hits $\{c, d\}$. Without loss of generality

$$
a_{i} \leqslant c \leqslant b_{i+1}
$$

in $P$. To see that $a_{2} \leqslant b_{3}$ also hits $\{c, d\}$ we first show that

$$
q_{2} \nless b_{3}^{T}
$$

in $T$. For this suppose $q_{2} \leqslant b_{3}^{T}$ in $T$. Then $q_{2}$ and $u_{3}$ are comparable in $T$, and thus $q_{2}<u_{3}$ in $T$ by (i). Since $q_{2}<b_{1}^{T}$ in $T$, it follows that $q_{2} \leqslant u_{3} \wedge b_{1}^{T}=q_{3}$ in $T$, contradicting our assumption that $q_{3}<q_{2}$ in $T$.

So we have $q_{2} \nless b_{3}^{T}$, and since $q_{3}<q_{2} \leqslant u_{2}<a_{2}^{T}$ in $T$, we deduce that the path connecting $a_{2}^{T}$ to $b_{3}^{T}$ in $T$ also includes the edge $\mathrm{p}\left(q_{2}\right) q_{2}$. Thus the relation $a_{2} \leqslant b_{3}$ indeed
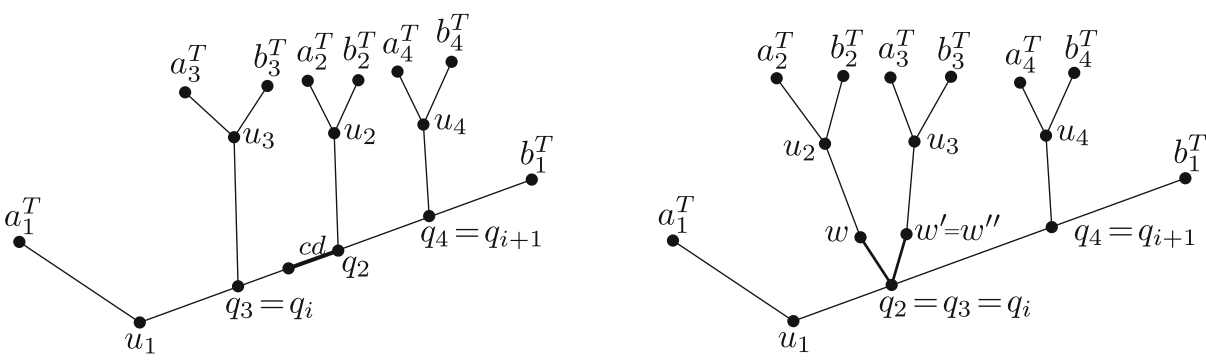

Fig. 12 Left: Situation in Claim 38 with $i=3$ (under the assumption that $q_{3}<q_{2}$ in $T$ ). Right: Possible situation in Claim 39 with $i=3$ (under the assumptions that $q_{2}=q_{3}$ and $w \neq w^{\prime}$ ) 
hits $\{c, d\}$. It cannot hit $c$ because otherwise $a_{2} \leqslant c \leqslant b_{i+1}$ (by (24)), which is not possible since $i \neq 2$. Hence we have

$$
a_{2} \leqslant d \leqslant b_{3}
$$

in $P$. Now, the relation $a_{0} \leqslant b_{2}$ clearly hits $\{c, d\}$ as well, but this is not possible as this implies $a_{i} \leqslant c \leqslant b_{2}$ (using (24)) or $a_{2} \leqslant d \leqslant b_{2}$ in $P$ (using (25)), a contradiction in both cases. This concludes the proof of (ii).

The following claim is a strengthening of Claim 38(ii).

Claim 39 Let $\Sigma \in \Sigma\left(v_{14}\right)$ and suppose that $\left\{\left(a_{i}, b_{i}\right)\right\}_{i=1}^{k}$ is a strict alternating cycle in $\operatorname{MM}\left(P, v_{14}, \Sigma\right)$ with root $\left(a_{1}, b_{1}\right)$. Let $u_{i}$ denote $u_{a_{i} b_{i}}$ and let $q_{i}:=u_{i} \wedge b_{1}^{T}$, for each $i \in\{1,2, \ldots, k\}$. Then

$$
u_{1}<q_{2}<q_{3}<b_{1}^{T} \text { in } T \text {. }
$$

Proof Using Claim 38(ii) we only need to show that $q_{2} \neq q_{3}$. Suppose to the contrary that we have $q_{2}=q_{3}$. Recall that $q_{2}<u_{2}$ in $T$, by Claim 37. By Claim 38(ii)we cannot have $u_{3}=q_{3}$ since $q_{2}=q_{3}$. Hence we also have $q_{3}<u_{3}$ in $T$.

Now, let $w$ be the neighbor of $q_{2}$ on the path from $q_{2}$ to $u_{2}$ in $T$, and let $w^{\prime}$ be the neighbor of $q_{3}$ on the path from $q_{3}=q_{2}$ to $u_{3}$ in $T$. Using that $q_{2}<u_{2}$ and $q_{3}<u_{3}$ in $T$ we deduce that

$$
q_{2}<w \leqslant u_{2} \quad \text { and } \quad q_{3}<w^{\prime} \leqslant u_{3}
$$

in $T$. Let us first suppose that $w=w^{\prime}$. Let $i$ be the largest index in $\{3,4, \ldots, k\}$ such that $w \leqslant u_{i}$ in $T$. We claim that

$$
w \nless b_{i+1}^{T}
$$

in $T$ (taking indices cyclically, as always). If $i<k$ this is because $w \leqslant b_{i+1}^{T}$ would imply $w \nless a_{i+1}^{T}$ (since $w \nless u_{i+1}$ in $T$ ), and thus $u_{i+1} \leqslant q_{2}$ in $T$, contradicting (i) of Claim 38. If $i=k$ this is because $w \leqslant b_{1}^{T}$ together with $w \leqslant u_{2}$ (by (26)) would imply $w \leqslant u_{2} \wedge b_{1}^{T}=$ $q_{2}$ in $T$, a contradiction.

Now, since $w \nless b_{i+1}^{T}$ in $T$ we deduce that the path from $a_{i}^{T}$ to $b_{i+1}^{T}$ includes the edge $q_{2} w$. Let $B(w) \cap B\left(q_{2}\right)=\{c, d\}$. Then the relations $a_{i} \leqslant b_{i+1}$ and $a_{1} \leqslant b_{2}$ both hit $\{c, d\}$, but not the same element (as otherwise $a_{i} \leqslant b_{2}$ in $P$, which cannot be since $i \neq 1$ ). Say we have

$$
a_{i} \leqslant c \leqslant b_{i+1} \text { and } a_{1} \leqslant d \leqslant b_{2}
$$

in $P$. Observe that the path from $r$ to $b_{i}^{T}$ in $T$ goes through the edge $q_{2} w$ as well, and hence $a_{0} \leqslant b_{i}$ also hits $\{c, d\}$. Thus $c \leqslant b_{i}$ or $d \leqslant b_{i}$ in $P$. In the first case we obtain $a_{i} \leqslant b_{i}$ and in the second $a_{1} \leqslant b_{i}$, a contradiction in each case. This closes the case $w=w^{\prime}$.

Finally, assume $w \neq w^{\prime}$. Let $B\left(q_{2}\right)=\{c, d, e\}$. Let $i$ be the largest index in $\{3,4, \ldots, k\}$ such that $q_{i}=q_{2}$. By Claim 38(ii) we know that $q_{i} \neq u_{i}$ (in particular $u_{i} \nless b_{1}^{T}$ in $T$ ), and thus $q_{2}=q_{i}<u_{i}$ in $T$. Let $w^{\prime \prime}$ be the neighbor of $q_{2}$ on the path from $q_{2}$ to $u_{i}$ in $T$. Note that we must have

$$
q_{2}<w^{\prime \prime} \leqslant u_{i} \quad \text { and } \quad w^{\prime \prime} \| b_{1}^{T}
$$

in $T$. Next we show that

$$
w^{\prime \prime} \nless b_{i+1}^{T}
$$

in $T$. Suppose that this is not true, and let us consider the case $i<k$ first. Then $w^{\prime \prime} \leqslant b_{i+1}^{T}$ would imply $w^{\prime \prime} \leqslant a_{i+1}^{T}$ as otherwise $u_{i+1} \leqslant q_{2}$ in $T$, contradicting (i) of Claim 38 . Moreover, this yields $w^{\prime \prime} \leqslant u_{i+1}$ in $T$. However, combined with the fact that $q_{2} \leqslant b_{1}^{T}$ and 
$w^{\prime \prime} \nless b_{1}^{T}$ in $T$ (by (27)) this implies $q_{i+1}=q_{2}$, contradicting the choice of $i$. If $i=k$ then $w^{\prime \prime} \leqslant b_{1}^{T}$ together with $w^{\prime \prime} \leqslant u_{i}$ (see (27)) would imply $w^{\prime \prime} \leqslant q_{i}$, again a contradiction.

So we indeed have $w^{\prime \prime} \nless b_{i+1}^{T}$ in $T$ (for an example illustrating this situation with $i=3$ see Fig. 12 on the right), and from this it follows that the $a_{i}^{T}-b_{i+1}^{T}$ path in $T$ includes the node $q_{2}$. Observe that so does the $a_{1}^{T}-b_{2}^{T}$ path (because $u_{1}<w_{1} \leqslant q_{2}<b_{2}^{T}$ in $T$ by Claim 36) and the $a_{2}^{T}-b_{3}^{T}$ path (because $w \neq w^{\prime}$ ). Hence the three relations $a_{1} \leqslant b_{2}, a_{2} \leqslant b_{3}$ and $a_{i} \leqslant b_{i+1}$ all hit $B\left(q_{2}\right)=\{c, d, e\}$. Clearly, no element in $B\left(q_{2}\right)$ is hit by two of these. In other words, each element of $B\left(q_{2}\right)$ is greater or equal to $a_{1}, a_{2}$, or $a_{i}$ in $P$.

Furthermore, the paths from $r$ to $b_{1}^{T}, b_{2}^{T}$ and $b_{3}^{T}$ in $T$ all include the edge $\mathrm{p}\left(q_{2}\right) q_{2}$. Hence two of the three relations $a_{0} \leqslant b_{1}, a_{0} \leqslant b_{2}, a_{0} \leqslant b_{3}$ hit the same element in $B\left(\mathrm{p}\left(q_{2}\right)\right) \cap$ $B\left(q_{2}\right) \varsubsetneqq\{c, d, e\}$. It follows that one element of the set $\left\{a_{1}, a_{2}, a_{i}\right\}$ is below two different elements of $\left\{b_{1}, b_{2}, b_{3}\right\}$ in $P$, which contradicts the assumption that the alternating cycle is strict. This concludes the proof.

Let $\Sigma \in \Sigma\left(v_{14}\right)$ and suppose that $\left\{\left(a_{i}, b_{i}\right)\right\}_{i=1}^{k}$ is a strict alternating cycle in $\operatorname{MM}\left(P, v_{14}, \Sigma\right)$ with root $\left(a_{1}, b_{1}\right)$. Let $u_{i}$ denote $u_{a_{i} b_{i}}$ for each $i \in\{1,2, \ldots, k\}$, and let $q_{2}:=u_{2} \wedge b_{1}^{T}$. In the following claims we will need to consider three specific neighbors of the node $q_{2}$ in $T$, namely, the neighbors of $q_{2}$ on the $q_{2}-u_{1}$ path, the $q_{2}-u_{2}$ path, and the $q_{2}-b_{1}^{T}$ path in $T$. Let us denote these nodes by $\mathrm{p}\left(q_{2}\right), \mathrm{m}\left(q_{2}\right)$ and $\mathrm{n}\left(q_{2}\right)$, respectively. By Claims 36 and 37, $\mathrm{p}\left(q_{2}\right), \mathrm{m}\left(q_{2}\right)$ and $\mathrm{n}\left(q_{2}\right)$ are well defined and distinct.

The following claim is illustrated in Fig. 13.

Claim 40 Let $\Sigma \in \Sigma\left(v_{14}\right)$ and suppose that $\left\{\left(a_{i}, b_{i}\right)\right\}_{i=1}^{k}$ is a strict alternating cycle in $\operatorname{MM}\left(P, v_{14}, \Sigma\right)$ with root $\left(a_{1}, b_{1}\right)$. Let $u_{i}$ denote $u_{a_{i} b_{i}}$ and let $q_{i}:=u_{i} \wedge b_{1}^{T}$, for each $i \in\{1,2, \ldots, k\}$. Then the elements of $B\left(q_{2}\right)$ can be written as $B\left(q_{2}\right)=\{c, d, e\}$ in such $a$ way that

(i) $B\left(q_{2}\right) \cap B\left(\mathrm{p}\left(q_{2}\right)\right)=\{c, d\}$;

(ii) $B\left(q_{2}\right) \cap B\left(\mathrm{~m}\left(q_{2}\right)\right)=\{c, e\}$;

(iii) $B\left(q_{2}\right) \cap B\left(\mathrm{n}\left(q_{2}\right)\right)=\{d, e\}$;

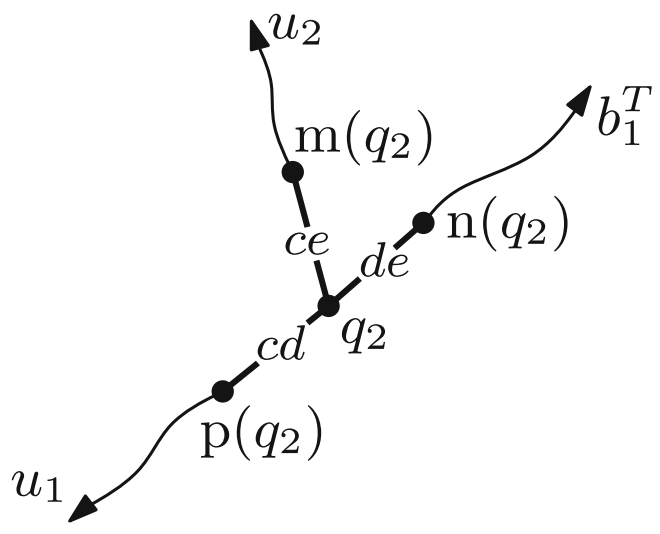

Fig. 13 Illustration of Claim 40 
and so that in $P$ we have

$$
\begin{aligned}
& a_{1} \leqslant c \leqslant b_{2} ; \\
& a_{0} \leqslant d \leqslant b_{1} ; \\
& a_{2} \leqslant e \leqslant b_{3} ;
\end{aligned}
$$

$a_{2} \nless c ;$

$a_{2} \nless d$.

Proof By Claims 36-39 we know that $u_{1}<w_{1} \leqslant q_{2}<u_{2}<\left\{a_{2}^{T}, b_{2}^{T}\right\}$ and $q_{2}<q_{3}<$ $\left\{b_{1}^{T}, a_{3}^{T}, b_{3}^{T}\right\}$ in $T$. Thus the $a_{1}^{T}-b_{2}^{T}$ path in $T$ goes through the nodes $\mathrm{p}\left(q_{2}\right), q_{2}$, and $\mathrm{m}\left(q_{2}\right)$; the $r-b_{1}^{T}$ path goes through $\mathrm{p}\left(q_{2}\right), q_{2}$, and $\mathrm{n}\left(q_{2}\right)$, and the $a_{2}^{T}-b_{3}^{T}$ path goes through $\mathrm{m}\left(q_{2}\right)$, $q_{2}$, and $\mathrm{n}\left(q_{2}\right)$. It follows that the corresponding three relations $a_{1} \leqslant b_{2}, a_{0} \leqslant b_{1}$ and $a_{2} \leqslant b_{3}$ in $P$ hit respectively the two sets $B\left(q_{2}\right) \cap B\left(\mathrm{p}\left(q_{2}\right)\right)$ and $B\left(q_{2}\right) \cap B\left(\mathrm{~m}\left(q_{2}\right)\right)$; the two sets $B\left(q_{2}\right) \cap B\left(\mathrm{p}\left(q_{2}\right)\right)$ and $B\left(q_{2}\right) \cap B\left(\mathrm{n}\left(q_{2}\right)\right)$, and the two sets $B\left(q_{2}\right) \cap B\left(\mathrm{~m}\left(q_{2}\right)\right)$ and $B\left(q_{2}\right) \cap$ $B\left(\mathrm{n}\left(q_{2}\right)\right)$. Clearly, no element of $B\left(q_{2}\right)$ is hit by two of these three relations. It follows that the elements of $B\left(q_{2}\right)$ can be written as $B\left(q_{2}\right)=\{c, d, e\}$ in such a way that properties (i)-(vi) hold. The remaining two properties (v) and (vi) are immediate consequences of these.

For each $\Sigma \in \Sigma\left(v_{14}\right)$ we define a corresponding directed graph $\hat{K}_{\Sigma}$ on the set $\operatorname{MM}\left(P, v_{14}, \Sigma\right)$ similarly as in Section 3.8: Given two distinct pairs $\left(a_{1}, b_{1}\right),\left(a_{2}, b_{2}\right) \in$ $\operatorname{MM}\left(P, v_{14}, \Sigma\right)$, there is an arc from $\left(a_{1}, b_{1}\right)$ to $\left(a_{2}, b_{2}\right)$ in $\hat{K}_{\Sigma}$ if and only if there is a strict alternating cycle $\left\{\left(a_{i}^{\prime}, b_{i}^{\prime}\right)\right\}_{i=1}^{k}$ in $\operatorname{MM}\left(P, v_{14}, \Sigma\right)$ with root $\left(a_{1}^{\prime}, b_{1}^{\prime}\right)$ which is such that $\left(a_{1}^{\prime}, b_{1}^{\prime}\right)=\left(a_{1}, b_{1}\right)$ and $\left(a_{2}^{\prime}, b_{2}^{\prime}\right)=\left(a_{2}, b_{2}\right)$. In the latter case, we say that the arc $f$ is induced by the strict alternating cycle $\left\{\left(a_{i}^{\prime}, b_{i}^{\prime}\right)\right\}_{i=1}^{k}$.

Note that there could possibly be different strict alternating cycles inducing the same arc in $\hat{K}_{\Sigma}$. Observe also that if $\left\{\left(a_{i}, b_{i}\right)\right\}_{i=1}^{k}$ is a strict alternating cycle inducing an arc in $\hat{K}_{\Sigma}$ then $\left(a_{1}, b_{1}\right)$ is always the root of the cycle (by the definition of 'inducing').

For each arc $f=\left(\left(a_{1}, b_{1}\right),\left(a_{2}, b_{2}\right)\right)$ of $\hat{K}_{\Sigma}$, define the corresponding three nodes of $T$ :

$$
\begin{aligned}
& u^{-}(f):=u_{a_{1} b_{1}} ; \\
& u^{+}(f):=u_{a_{2} b_{2}} ; \\
& q(f):=u_{a_{2} b_{2}} \wedge b_{1}^{T} .
\end{aligned}
$$

Observe that

$$
u^{-}(f)<w_{a_{1} b_{1}} \leqslant q(f)<u^{+}(f)
$$

in $T$ by Claims 36 and 37 . This will be used repeatedly in what follows.

Claim 41 For each $\Sigma \in \Sigma\left(v_{14}\right)$, any two arcs $f, g$ in $\hat{K}_{\Sigma}$ sharing the same source satisfy $q(f)=q(g)$.

Proof Assume to the contrary that $q(f) \neq q(g)$. Let $\left(a_{1}, b_{1}\right) \in \operatorname{MM}\left(P, v_{14}, \Sigma\right)$ denote the source of the two arcs $f$ and $g$. By definition $q(f)<b_{1}^{T}$ and $q(g)<b_{1}^{T}$ in $T$. Thus in particular $q(f)$ and $q(g)$ are comparable in $T$, say without loss of generality $q(f)<q(g)$. Hence we have $u_{a_{1} b_{1}}<w_{a_{1} b_{1}} \leqslant q(f)<q(g)<b_{1}^{T}$ in $T$.

Let $\left(a_{2}, b_{2}\right),\left(a_{2}^{\prime}, b_{2}^{\prime}\right) \in \operatorname{MM}\left(P, v_{14}, \Sigma\right)$ denote the targets of arcs $f$ and $g$, respectively. Let $\left(a_{3}, b_{3}\right), \ldots,\left(a_{k}, b_{k}\right) \in \operatorname{MM}\left(P, v_{14}, \Sigma\right)$ be such that $\left\{\left(a_{i}, b_{i}\right)\right\}_{i=1}^{k}$ is a strict alternating cycle inducing $f$. Write the elements of $B(q(f))$ as $B(q(f))=\{c, d, e\}$ as in Claim 40 when applied to the latter cycle. Then the paths from $a_{1}^{T}$ to $b_{2}^{\prime T}$ and from $r$ to $b_{1}^{T}$ in $T$ both include the three nodes $\mathrm{p}(q(f)), q(f)$, and $\mathrm{n}(q(f))$. Hence, the two relations $a_{1} \leqslant b_{2}^{\prime}$ 
and $a_{0} \leqslant b_{1}$ in $P$ both hit the two sets $B(q(f)) \cap B(\mathrm{p}(q(f)))=\{c, d\}$ and $B(q(f)) \cap$ $B(\mathrm{n}(q(f)))=\{d, e\}$. On the other hand, each of $c, d, e$ is clearly hit by at most one of these two relations. It follows that one relation hits $d$ and the other hits both $c$ and $e$,implying $c \leqslant e$ in $P$ by Observation 10. However, this contradicts $c \| e$ in $P$ (cf. property (v) of Claim 40).

The following claim is similar to the previous one.

Claim 42 For each $\Sigma \in \Sigma\left(v_{14}\right)$, any two arcs $f, g$ in $\hat{K}_{\Sigma}$ sharing the same target satisfy $q(f)=q(g)$.

Proof Assume to the contrary that $q(f) \neq q(g)$. Let $\left(a_{2}, b_{2}\right) \in \operatorname{MM}\left(P, v_{14}, \Sigma\right)$ denote the common target of the two arcs $f$ and $g$. We have $q(f) \leqslant u_{a_{2} b_{2}}$ and $q(g) \leqslant u_{a_{2} b_{2}}$ in $T$. Thus $q(f)$ and $q(g)$ are comparable in $T$, say $q(f)<q(g)$ in $T$.

Applying Claim 40 on a strict alternating cycle inducing $f$, we see that there exists an element $e \in B(q(f))$ such that $a_{2} \leqslant e$ in $P$. Since $q(f)<q(g) \leqslant u_{a_{2} b_{2}}<a_{2}^{T}$ in $T$, the path from $q(f)$ to $a_{2}^{T}$ in $T$ goes through $\mathrm{p}(q(g))$ and $q(g)$. Thus the relation $a_{2} \leqslant e$ hits $B(q(g)) \cap B(\mathrm{p}(q(g)))$, and hence $a_{2} \leqslant s$ in $P$ for some $s \in B(q(g)) \cap B(\mathrm{p}(q(g)))$. However, applying Claim 40 on a strict alternating cycle inducing $g$ this time, we deduce that $a_{2} \nless$ in $P$ for each $t \in B(q(g)) \cap B(\mathrm{p}(q(g)))$ (cf. property (vi)), and therefore in particular $a_{2} \nless s$ in $P$, a contradiction.

Claim 43 For each $\Sigma \in \Sigma\left(v_{14}\right)$ and any two arcs $f, g$ in $\hat{K}_{\Sigma}$, we neither have

$$
q(f)<q(g)<u^{+}(f) \leqslant u^{+}(g)
$$

nor

$$
q(f)<q(g)<u^{+}(g) \leqslant u^{+}(f)
$$

in $T$.

Proof Let $\left(a_{2}, b_{2}\right)$ and $\left(a_{2}^{\prime}, b_{2}^{\prime}\right)$ denote the targets of $f$ and $g$, respectively. Arguing by contradiction, assume that at least one of the two inequalities holds. Then we have

$$
q(f) \leqslant \mathrm{p}(q(g))<q(g)<\mathrm{m}(q(g)) \leqslant\left\{u_{a_{2} b_{2}}, u_{a_{2}^{\prime} b_{2}^{\prime}}\right\}
$$

in $T$.

Now consider a strict alternating cycle inducing $g$ and write the elements of $B(q(g))$ as $B(q(g))=\{c, d, e\}$ as in Claim 40 when applied to the latter cycle. See Fig. 14 for an illustration.

By this claim we have

$$
c \leqslant b_{2}^{\prime} \text { and } a_{2}^{\prime} \leqslant e \text { and } e \nless d
$$

in $P$. Applying Claim 40 on a strict alternating cycle inducing $f$, we also deduce that there exists $s \in B(q(f))$ such that $a_{2} \leqslant s$ in $P$.

Given that by (28) the path from $a_{2}^{T}$ to $q(f)$ goes first through $u_{a_{2} b_{2}}$ and then through the three nodes $\mathrm{m}(q(g)), q(g)$ and $\mathrm{p}(q(g))$ in $T$, it follows that the relation $a_{2} \leqslant s$ hits both $B(q(g)) \cap B(\mathrm{~m}(q(g)))=\{c, e\}$ and $B(q(g)) \cap B(\mathrm{p}(q(g)))=\{c, d\}$. If it did not hit $c$, then it would hit both $d$ and $e$, and we would have $e \leqslant d$ in $P$ by Observation 10, which is not possible by (29). Thus $a_{2} \leqslant s$ hits $c$, that is, $a_{2} \leqslant c \leqslant s$ in $P$. Together with (29) this implies

$$
a_{2} \leqslant c \leqslant b_{2}^{\prime}
$$

in $P$, and we also deduce $\left(a_{2}, b_{2}\right) \neq\left(a_{2}^{\prime}, b_{2}^{\prime}\right)$. 


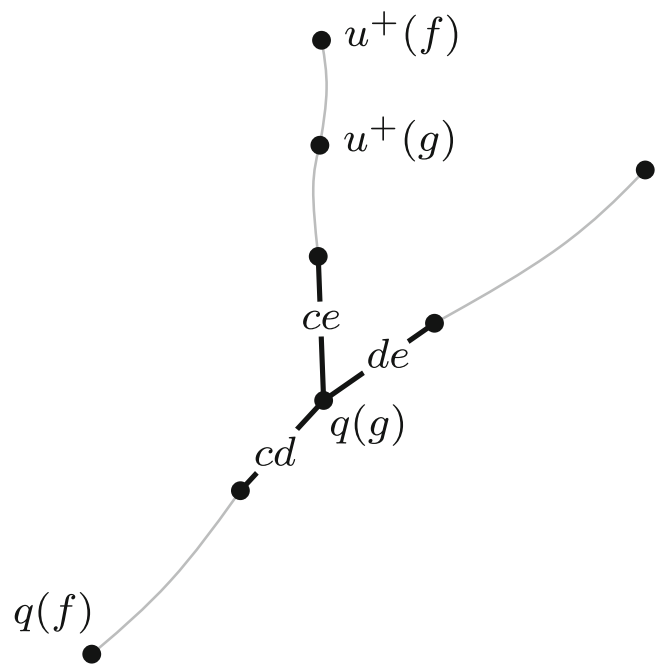

Fig. 14 Illustration of the proof of Claim 43

Now, the path from $r$ to $b_{2}^{T}$ in $T$ goes through $q(g)$ and $\mathrm{m}(q(g))$, and thus $a_{0} \leqslant b_{2}$ hits $\{c, e\}$. It cannot hit $c$, as otherwise $a_{2} \leqslant c \leqslant b_{2}$ in $P$. Hence $a_{0} \leqslant b_{2}$ hits $e$, and we have $a_{0} \leqslant e \leqslant b_{2}$ in $P$, which by (29) implies

$$
a_{2}^{\prime} \leqslant e \leqslant b_{2} .
$$

It follows that $\left(a_{2}, b_{2}\right),\left(a_{2}^{\prime}, b_{2}^{\prime}\right)$ is an alternating cycle of length 2 , which is a contradiction since there is no such cycle in $\operatorname{MM}\left(P, v_{14}, \Sigma\right)$.

For the next claim let us recall that given a pair $(a, b) \in \operatorname{MM}\left(P, v_{14}, \Sigma\right)$, the elements of $B\left(u_{a b}\right)$ are labeled with $x_{a b}, y_{a b}, z_{a b}$, and it holds that $B\left(u_{a b}\right) \cap B\left(\mathrm{p}\left(u_{a b}\right)\right)=\left\{x_{a b}, y_{a b}\right\}$,

$$
a \leqslant x_{a b} \nless b, \quad \text { and } a \nless y_{a b} \leqslant b
$$

in $P$.

Claim 44 For each $\Sigma \in \Sigma\left(v_{14}\right)$, no two arcs $f$, $g$ in $\hat{K}_{\Sigma}$ satisfy

$$
u^{-}(f) \leqslant q(g)<u^{+}(g) \leqslant q(f) .
$$

in $T$.

Proof Assume to the contrary that the inequality holds. Let $\left\{\left(a_{i}, b_{i}\right)\right\}_{i=1}^{k}$ be a strict alternating cycle inducing $f$ and let $\left\{\left(a_{i}^{\prime}, b_{i}^{\prime}\right)\right\}_{i=1}^{\ell}$ be one inducing $g$. Let $u_{i}:=u_{a_{i} b_{i}}, q_{i}:=$ $u_{a_{i} b_{i}} \wedge b_{1}^{T}, x_{i}:=x_{a_{i} b_{i}}, y_{i}:=y_{a_{i} b_{i}}$, and $z_{i}:=z_{a_{i} b_{i}}$ for each $i \in\{1, \ldots, k\}$, and let $u_{i}^{\prime}:=u_{a_{i}^{\prime} b_{i}^{\prime}}, q_{i}^{\prime}:=u_{a_{i}^{\prime} b_{i}^{\prime}} \wedge b_{1}^{\prime T}, x_{i}^{\prime}:=x_{a_{i}^{\prime} b_{i}^{\prime}}, y_{i}^{\prime}:=y_{a_{i}^{\prime} b_{i}^{\prime}}$, and $z_{i}^{\prime}:=z_{a_{i}^{\prime} b_{i}^{\prime}}$ for each $i \in\{1, \ldots, \ell\}$. Thus $u^{-}(f)=u_{1}, q(f)=q_{2}, u^{+}(g)=u_{2}^{\prime}, q(g)=q_{2}^{\prime}$, and

$$
u_{1} \leqslant q_{2}^{\prime}<u_{2}^{\prime} \leqslant q_{2}<\left\{b_{1}^{T}, b_{2}^{T}\right\}
$$

in $T$ by our assumption. See Fig. 15 for an illustration of the situation.

The $a_{1}^{T}-b_{2}^{T}$ path and the $r-b_{1}^{T}$ path both go through $\mathrm{p}\left(u_{2}^{\prime}\right)$ and $u_{2}^{\prime}$ in $T$. Thus the two relations $a_{1} \leqslant b_{2}$ and $a_{0} \leqslant b_{1}$ both hit $B\left(\mathrm{p}\left(u_{2}^{\prime}\right)\right) \cap B\left(u_{2}^{\prime}\right)=\left\{x_{2}^{\prime}, y_{2}^{\prime}\right\}$, and clearly neither 
of $x_{2}^{\prime}, y_{2}^{\prime}$ is hit by both relations. We cannot have $a_{1} \leqslant y_{2}^{\prime} \leqslant b_{2}$ and $a_{0} \leqslant x_{2}^{\prime} \leqslant b_{1}$ in $P$, because otherwise we would have $a_{1} \leqslant y_{2}^{\prime} \leqslant b_{2}^{\prime}$ and $a_{2}^{\prime} \leqslant x_{2}^{\prime} \leqslant b_{1}$ in $P$, implying that $\left(a_{1}, b_{1}\right),\left(a_{2}^{\prime}, b_{2}^{\prime}\right)$ is an alternating cycle of length 2 in $\operatorname{MM}\left(P, v_{14}, \Sigma\right)$, a contradiction. Hence we have

$$
a_{1} \leqslant x_{2}^{\prime} \leqslant b_{2} \quad \text { and } \quad a_{0} \leqslant y_{2}^{\prime} \leqslant b_{1}
$$

in $P$.

Let us denote the elements in $B\left(q_{2}^{\prime}\right)$ as $B\left(q_{2}^{\prime}\right)=\{c, d, e\}$ as in Claim 40 when applied to the strict alternating cycle $\left\{\left(a_{i}^{\prime}, b_{i}^{\prime}\right)\right\}_{i=1}^{\ell}$. Then we have $a_{0} \leqslant d \leqslant b_{1}^{\prime}$ in $P$, as well as $a_{2}^{\prime} \leqslant$ $e \leqslant b_{3}^{\prime}$ and $a_{1}^{\prime} \leqslant c \leqslant b_{2}^{\prime}$. Given that the relation $a_{2}^{\prime} \leqslant e$ hits $B\left(\mathrm{p}\left(u_{2}^{\prime}\right)\right) \cap B\left(u_{2}^{\prime}\right)=\left\{x_{2}^{\prime}, y_{2}^{\prime}\right\}$, and that it clearly cannot hit $y_{2}^{\prime}$ because $y_{2}^{\prime} \leqslant b_{2}^{\prime}$ in $P$, we have $a_{2}^{\prime} \leqslant x_{2}^{\prime} \leqslant e$ in $P$. Similarly, $c \leqslant b_{2}^{\prime}$ hits $\left\{x_{2}^{\prime}, y_{2}^{\prime}\right\}$ as well and cannot hit $x_{2}^{\prime}$ because $a_{2}^{\prime} \leqslant x_{2}^{\prime}$ in $P$, hence $c \leqslant y_{2}^{\prime} \leqslant b_{2}^{\prime}$ in $P$. Summarizing, we have

$$
\begin{array}{r}
a_{2}^{\prime} \leqslant x_{2}^{\prime} \leqslant e \leqslant b_{3}^{\prime}, \\
a_{1}^{\prime} \leqslant c \leqslant y_{2}^{\prime} \leqslant b_{2}^{\prime}, \\
a_{0} \leqslant d \leqslant b_{1}^{\prime}
\end{array}
$$

in $P$.

Recall that $u_{1} \leqslant q_{2}^{\prime}$ in $T$ by (30). We split the rest of the argument into two cases and start with the case that $u_{1}<q_{2}^{\prime}$ in $T$. Then the path from $a_{1}^{T}$ to $u_{2}^{\prime}$ goes through $\mathrm{p}\left(q_{2}^{\prime}\right)$ and $q_{2}^{\prime}$. It follows that the relation $a_{1} \leqslant x_{2}^{\prime}$ hits $B\left(\mathrm{p}\left(q_{2}^{\prime}\right)\right) \cap B\left(q_{2}^{\prime}\right)=\{c, d\}$. If it hits $c$ then $c \leqslant x_{2}^{\prime}$ in $P$, which implies $a_{1}^{\prime} \leqslant c \leqslant x_{2}^{\prime} \leqslant e \leqslant b_{3}^{\prime}$ by (33) and (32), a contradiction to the assumption that we deal with a strict alternating cycle of length at least 3 . Hence, we have $a_{1} \leqslant d \leqslant x_{2}^{\prime}$. However, using (34) we obtain $a_{1} \leqslant d \leqslant b_{1}^{\prime}$ in $P$, and since $a_{1}^{\prime} \leqslant c \leqslant y_{2}^{\prime} \leqslant b_{1}$ in $P$ (by combining (33) and (31)) this implies that $\left(a_{1}, b_{1}\right) \neq\left(a_{1}^{\prime}, b_{1}^{\prime}\right)$ and therefore that $\left(a_{1}, b_{1}\right),\left(a_{1}^{\prime}, b_{1}^{\prime}\right)$ is an alternating cycle of length 2 in $\operatorname{MM}\left(P, v_{14}, \Sigma\right)$, a contradiction.

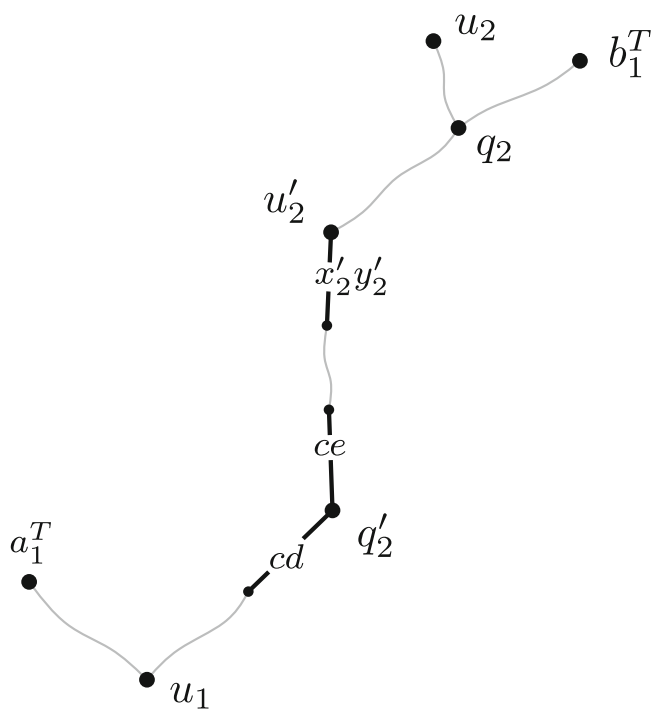

Fig. 15 Illustration of the proof of Claim 44 
It remains to consider the case that $u_{1}=q_{2}^{\prime}$ in $T$. Then the path from $a_{1}^{T}$ to $u_{2}^{\prime}$ in $T$ goes through $q_{2}^{\prime}$ and $\mathrm{m}\left(q_{2}^{\prime}\right)$. Thus $a_{1} \leqslant x_{2}^{\prime}$ hits $B\left(q_{2}^{\prime}\right) \cap B\left(\mathrm{~m}\left(q_{2}^{\prime}\right)\right)=\{c, e\}$. The relation $a_{1} \leqslant x_{2}^{\prime}$ cannot hit $c$, for the same reason as in the previous paragraph. Hence $a_{1} \leqslant e \leqslant x_{2}^{\prime}$ in $P$, implying that $x_{2}^{\prime}=e$ by (32).

Now, observe that $e \in B\left(u_{1}\right)$ since $u_{1}=q_{2}^{\prime}$. Given that $e \notin B\left(q_{2}^{\prime}\right) \cap B\left(\mathrm{p}\left(q_{2}^{\prime}\right)\right)=\{c, d\}=$ $B\left(u_{1}\right) \cap B\left(\mathrm{p}\left(u_{1}\right)\right)=\left\{x_{1}, y_{1}\right\}$, we conclude $x_{2}^{\prime}=e=z_{1}$. However, in the coloring $\phi$ we have $\phi\left(x_{2}^{\prime}\right)=\phi\left(x_{1}\right) \neq \phi\left(z_{1}\right)$ since $\alpha_{11}\left(a_{1}, b_{1}\right)=\alpha_{11}\left(a_{2}^{\prime}, b_{2}^{\prime}\right)$, contradicting $x_{2}^{\prime}=z_{1}$. This concludes the proof.

Claim 45 Let $\Sigma \in \Sigma\left(v_{14}\right)$ and suppose that $f_{1}, f_{2}, g_{1}, g_{2}$ are arcs of $\hat{K}_{\Sigma}$ satisfying

- $u^{+}\left(f_{1}\right)=u^{-}\left(f_{2}\right)$

- $u^{+}\left(g_{1}\right)=u^{-}\left(g_{2}\right)$

- $q\left(f_{2}\right)=q\left(g_{2}\right)$.

Then it also holds that $q\left(f_{1}\right)=q\left(g_{1}\right)$.

Proof Recall that $u^{-}(f)<q(f)<u^{+}(f)$ for every arc $f$ of $\hat{K}_{\Sigma}$ (by Claims 36 and 37). It follows from the assumptions that

$$
q\left(g_{1}\right)<u^{+}\left(g_{1}\right)=u^{-}\left(g_{2}\right)<q\left(g_{2}\right)
$$

and

$$
q\left(f_{1}\right)<u^{+}\left(f_{1}\right)=u^{-}\left(f_{2}\right)<q\left(f_{2}\right)
$$

in $T$. Thus $q\left(g_{1}\right)$ and $q\left(f_{1}\right)$ are comparable in $T$. Arguing by contradiction, suppose that $q\left(f_{1}\right) \neq q\left(g_{1}\right)$. Using symmetry, we may assume without loss of generality $q\left(f_{1}\right)<q\left(g_{1}\right)$ in $T$.

Since $q\left(g_{1}\right)<q\left(g_{2}\right)=q\left(f_{2}\right)$ by (35) and $u^{+}\left(f_{1}\right)=u^{-}\left(f_{2}\right)<q\left(f_{2}\right)$ in $T$ by (36), the two nodes $q\left(g_{1}\right)$ and $u^{+}\left(f_{1}\right)$ are also comparable in $T$.

First suppose that $q\left(g_{1}\right)<u^{+}\left(f_{1}\right)$ in $T$. Then observe that the two nodes $u^{+}\left(g_{1}\right)$ and $u^{+}\left(f_{1}\right)$ are comparable in $T$ since $u^{+}\left(g_{1}\right)=u^{-}\left(g_{2}\right)<q\left(g_{2}\right)=q\left(f_{2}\right)$ and $u^{+}\left(f_{1}\right)<q\left(f_{2}\right)$ in $T$ (by (35) and (36)). Hence we have $q\left(f_{1}\right)<q\left(g_{1}\right)<u^{+}\left(f_{1}\right) \leqslant u^{+}\left(g_{1}\right)$ or $q\left(f_{1}\right)<$ $q\left(g_{1}\right)<u^{+}\left(g_{1}\right) \leqslant u^{+}\left(f_{1}\right)$ in $T$, neither of which is possible by Claim 43, a contradiction.

Next, assume that $q\left(g_{1}\right) \geqslant u^{+}\left(f_{1}\right)$ in $T$. We immediately obtain $u^{-}\left(f_{2}\right)=u^{+}\left(f_{1}\right) \leqslant$ $q\left(g_{1}\right)<u^{+}\left(g_{1}\right)<q\left(f_{2}\right)$ in $T$, which is forbidden by Claim 44 , again a contradiction.

Claim 46 The graph $\hat{K}_{\Sigma}$ is bipartite for each $\Sigma \in \Sigma\left(v_{14}\right)$.

Proof Suppose that there is an odd cycle $C=\left\{\left(a_{i}, b_{i}\right)\right\}_{i=1}^{k}$ in the undirected graph underlying $\hat{K}_{\Sigma}$. (Thus $C$ is not necessarily a directed cycle.) For each $i \in\{1, \ldots, k\}$, let $f_{i}$ be an arc between $\left(a_{i}, b_{i}\right)$ and $\left(a_{i+1}, b_{i+1}\right)$ in $\hat{K}_{\Sigma}$, where indices are taken cyclically as always. If $f_{i}=\left(\left(a_{i}, b_{i}\right),\left(a_{i+1}, b_{i+1}\right)\right)$, that is, $\left(a_{i}, b_{i}\right)$ is the source of $f_{i}$, we say that $f_{i}$ goes forward, while if $f_{i}=\left(\left(a_{i+1}, b_{i+1}\right),\left(a_{i}, b_{i}\right)\right)$ we say that $f_{i}$ goes backward.

We define a cyclically ordered sequence $S$ of arcs in $\left\{f_{1}, f_{2}, \ldots, f_{k}\right\}$ as follows. We start with $S=\left(f_{1}, \ldots, f_{k}\right)$. It will be convenient to say that we go along $S$ in clockwise order whenver we use the forward direction in $S$ (to not mix it up with forward and backward edges). Now, we repeat the following modification until it is no longer possible: If $S$ has size at least 5 and there are two (cyclically) consecutive $\operatorname{arcs} f, f^{\prime}$ in clockwise order in $S$ with $f$ going forward and $f^{\prime}$ going backward then remove both $f$ and $f^{\prime}$ from $S$. 
By construction, the resulting sequence $S$ has the following property: Either $S$ contains at least five arcs and all arcs go in the same direction, or $S$ contains exactly three arcs.

We claim that during the above iterative process the cyclic sequence $S$ fulfills the following invariants at all times: For any two consecutive arcs $f$ and $f^{\prime}$ in clockwise order in $S$,

(i) if $f$ and $f^{\prime}$ both go forward then $q(f)<q\left(f^{\prime}\right)$ in $T$, while if $f$ and $f^{\prime}$ both go backward then $q(f)>q\left(f^{\prime}\right)$ in $T$;

(ii) if $f$ and $f^{\prime}$ go in the same direction then there exist $\operatorname{arcs} g, g^{\prime}$ in $\hat{K}_{\Sigma}$ such that

- $q(g)=q(f)$

- $q\left(g^{\prime}\right)=q\left(f^{\prime}\right)$, and

- $u^{+}(g)=u^{-}\left(g^{\prime}\right)$ if $f$ and $f^{\prime}$ go forward, $u^{-}(g)=u^{+}\left(g^{\prime}\right)$ otherwise, and

(iii) if $f$ and $f^{\prime}$ go in opposite directions then $q(f)=q\left(f^{\prime}\right)$.

Note that (ii) implies (i). Indeed, suppose $f$ and $f^{\prime}$ go forward (for the backward direction the argument is analogous). Then take arcs $g$ and $g^{\prime}$ witnessing (ii). We have $q(f)=q(g)<u^{+}(g)=u^{-}\left(g^{\prime}\right)<q\left(g^{\prime}\right)=q\left(f^{\prime}\right)$. (Recall that $u^{-}(g)<q(g)<u^{+}(g)$ for every arc $g$ of $\hat{K}_{\Sigma}$ by Claims 36 and 37.)

First, we prove that the invariants hold at the beginning of the process, so for the sequence $\left(f_{1}, \ldots, f_{k}\right)$. In order to prove (ii), for each $i \in\{1, \ldots, k\}$ take $g:=f_{i}$ and $g^{\prime}:=f_{i+1}$. Then clearly (ii)holds, and property (iii) follows from Claims 41 and 42.

Next we show that the invariants hold after each modification step. Consider thus the sequence $S$ just before a modification step, and suppose that $S$ satisfied the required properties. Let $f^{0}, f^{1}, f^{2}, f^{3}$ be the four consecutive arcs in $S$ in clockwise order which are such that $f^{1}$ goes forward and $f^{2}$ goes backward. After removing $f^{1}$ and $f^{2}$, the arcs $f^{0}$ and $f^{3}$ will become consecutive in $S$ (in clockwise order). We only need to establish the invariants for the consecutive pair $f^{0}, f^{3}$, since all other consecutive pairs already satisfy them by assumption.

Let us start with the case that $f^{0}$ and $f^{3}$ both go forward. Since $f^{1}, f^{2}$ and $f^{3}$ alternate in directions, we get $q\left(f^{1}\right)=q\left(f^{2}\right)=q\left(f^{3}\right)$ by (iii). By (ii) and the fact that $f^{0}$ and $f^{1}$ go forward, there are arcs $g^{0}, g^{1}$ in $\hat{K}_{\Sigma}$ such that $q\left(g^{0}\right)=q\left(f^{0}\right), q\left(g^{1}\right)=q\left(f^{1}\right)$ and $u^{+}\left(g^{0}\right)=u^{-}\left(g^{1}\right)$. Now, since $q\left(g^{1}\right)=q\left(f^{1}\right)=q\left(f^{3}\right)$, the arcs $g^{0}, g^{1}$ also fulfill the conditions of (ii) for $f^{0}$ and $f^{3}$.

The case that both $f^{0}$ and $f^{3}$ go backward is symmetric to the previous one and is thus omitted.

Next, suppose that $f^{0}$ goes forward and $f^{3}$ goes backward. Here we have to show that (iii) holds for $f^{0}$ and $f^{3}$. Since $f^{0}$ and $f^{1}$ both go forward and $f^{2}$ and $f^{3}$ both go backward, by (ii) there are arcs $g^{0}, g^{1}$ and $g^{2}, g^{3}$ in $\hat{K}_{\Sigma}$ such that $q\left(g^{j}\right)=q\left(f^{j}\right)$ for each $j \in$ $\{0,1,2,3\}, u^{+}\left(g^{0}\right)=u^{-}\left(g^{1}\right)$, and $u^{-}\left(g^{2}\right)=u^{+}\left(g^{3}\right)$. Using (iii) we deduce that $q\left(g^{1}\right)=$ $q\left(f^{1}\right)=q\left(f^{2}\right)=q\left(g^{2}\right)$. Applying Claim 45 on the arcs $g^{0}, g^{1}, g^{3}, g^{2}$ (in this order), we conclude $q\left(f^{0}\right)=q\left(g^{0}\right)=q\left(g^{3}\right)=q\left(f^{3}\right)$, as desired.

Finally, assume that $f^{0}$ goes backward and $f^{3}$ goes forward. Again we have show that (iii) holds for $f^{0}, f^{3}$. But in this case the four directions of $f^{0}, f^{1}, f^{2}, f^{3}$ alternate. It follows that $q\left(f^{0}\right)=q\left(f^{1}\right)=q\left(f^{2}\right)=q\left(f^{3}\right)$ by (iii).

Now that the above invariants of $S$ have been established, let us go back to the final sequence $S$ resulting from the modification process. We claim that there are always two consecutive arcs going in opposite directions in $S$. Indeed, if not then they either all go forward or all go backward. In the first case $q(f)<q\left(f^{\prime}\right)$ in $T$ for any two consecutive 
arcs $f, f^{\prime}$ in clockwise order in $S$ by (i), while in the second case $q(f)>q\left(f^{\prime}\right)$ in $T$ for any two such arcs $f, f^{\prime}$. However, neither of these two situations can occur in a circular sequence.

This shows in particular that the modification process results in a sequence $S$ of size 3, say $S=\left(f^{1}, f^{2}, f^{3}\right)$. We may suppose without loss of generality that $f^{1}$ and $f^{2}$ go in the same direction and $f^{3}$ in the other (since the sequence $S$ can always be shifted cyclically to ensure this property). This implies $q\left(f^{1}\right) \neq q\left(f^{2}\right)$ by (i), $q\left(f^{2}\right)=q\left(f^{3}\right)$ by (i), and $q\left(f^{3}\right)=q\left(f^{1}\right)$ by (iii). This is a contradiction, which concludes the proof.

Using Claim 46 we let $\psi_{14, \Sigma}: \operatorname{MM}\left(P, v_{14}, \Sigma\right) \rightarrow\{1,2\}$ be a 2-coloring of $\hat{K}_{\Sigma}$, for each $\Sigma \in \Sigma\left(v_{14}\right)$. The function $\alpha_{14}$ then records the color of a pair in this coloring:

For each $\Sigma \in \boldsymbol{\Sigma}\left(\nu_{14}\right)$ and each pair $(a, b) \in \operatorname{MM}\left(P, \nu_{14}, \Sigma\right)$, we let

$$
\alpha_{14}(a, b):=\psi_{14, \Sigma}(a, b) .
$$

\subsection{Fourth leaf of $\Psi$ : Node $v_{15}$}

It remains to verify that for each $\Sigma \in \Sigma\left(v_{15}\right)$ the set $\operatorname{MM}\left(P, v_{15}, \Sigma\right)$ is reversible. Recall that $\alpha_{13}$ ensures that there are no 2-cycles in $\operatorname{MM}\left(P, v_{15}, \Sigma\right)$ and that $\alpha_{14}$ ensures that there are no strict alternating cycles of length at least 3 in $\operatorname{MM}\left(P, v_{15}, \Sigma\right)$. It follows that $\operatorname{MM}\left(P, v_{15}, \Sigma\right)$ is reversible.

This concludes the proof of Theorem 7 .

Acknowledgments This research was initiated during the workshop Order and Geometry held at the Technische Universität Berlin in August 2013. We are grateful to the organizers and the other participants for providing a very stimulative research environment. We also thank Grzegorz Gutowski and Tomasz Krawczyk for many fruitful discussions at the early stage of this project. Finally, we are much grateful to the referees for their many helpful remarks and suggestions, which greatly improved the readability of the paper.

Open Access This article is distributed under the terms of the Creative Commons Attribution 4.0 International License (http://creativecommons.org/licenses/by/4.0/), which permits unrestricted use, distribution, and reproduction in any medium, provided you give appropriate credit to the original author(s) and the source, provide a link to the Creative Commons license, and indicate if changes were made.

\section{References}

1. Biró, C., Keller, M.T., Young, S.J.: Posets with cover graph of pathwidth two have bounded dimension. Order, in press, doi:10.1007/s11083-015-9359-7. arXiv:1308.4877

2. Eppstein, D.: Diameter and treewidth in minor-closed graph families. Algorithmica 27(3-4), 275-291 (2000)

3. Felsner, S., Trotter, W.T., Wiechert, V.: The dimension of posets with planar cover graphs. Graphs Combin. 31(4), 927-939 (2015)

4. Joret, G., Micek, P., Milans, K.G., Trotter, W.T., Walczak, B., Wang, R.: Tree-width and dimension. Combinatorica, in press, doi:10.1007/s00493-014-3081-8. arXiv:1301.5271

5. Joret, G., Micek, P., Wiechert, V.: Sparsity and dimension. In: proceedings of the Twenty-Seventh Annual ACM-SIAM Symposium on Discrete Algorithms, pp. 1804-1813 (2016). arXiv:1507.01120

6. Kelly, D.: On the dimension of partially ordered sets. Discret. Math. 35, 135-156 (1981)

7. Micek, P., Wiechert, V.: Topological minors of cover graphs and dimension. Submitted, arXiv: 1504.07388 
8. Streib, N., Trotter, W.T.: Dimension and height for posets with planar cover graphs. Eur. J. Combin. 35, 474-489 (2014)

9. Trotter, W.illiam.T.: Combinatorics and partially ordered sets. Dimension theory. Johns Hopkins Series in the Mathematical Sciences. Johns Hopkins University Press, Baltimore, MD (1992)

10. Trotter, W.T.: Partially ordered sets. In: Handbook of Combinatorics, vol. 1,2, pp. 433-480. Elsevier Science B.V., Amsterdam (1995)

11. Trotter, W.T., Jr., Moore, J.I., Jr.: The dimension of planar posets. J. Comb. Theory Ser. B 22(1), 54-67 (1977)

12. Walczak, B.: Minors and dimension. In: Proceedings of the Twenty-Sixth Annual ACM-SIAM Symposium on Discrete Algorithms, pp. 1698-1707 (2015). arXiv:1407.4066 